\title{
THE PRECLINICAL STUDY OF THE EXPRESSION CHANGES OF PGC-1 $\alpha$ AND SIRT GENES
}

\author{
Ph.D. THESIS
}

ANDRÁS SALAMON, M.D.

SZEGED

2020 


\title{
THE PRECLINICAL STUDY OF THE EXPRESSION CHANGES OF PGC-1 $\alpha$ AND SIRT GENES
}

\author{
Ph.D. THESIS
}

ANDRÁS SALAMON, M.D.

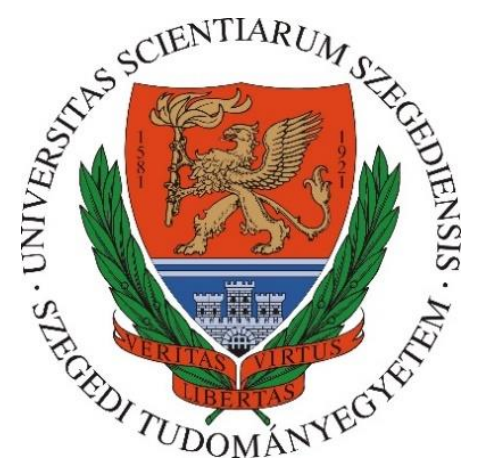

CLINICAL AND EXPERIMENTAL NEUROSCIENCE PROGRAM DOCTORAL SCHOOL OF CLINICAL MEDICINE

FACULTY OF MEDICINE UNIVERSITY OF SZEGED

\author{
SUPERVISORS: \\ PÉTER KLIVÉNYI, M.D., Ph.D., D.Sc. \\ DÉNES ZÁDORI, M.D., Ph.D.
}

SZEGED

2020 


\section{PUBLICATIONS DIRECTLY RELATED TO THE THESIS}

I. Salamon A, Maszlag-Török R, Veres G, Boros FA, Vágvölgyi-Sümegi E, Somogyi A, Vécsei L, Klivényi P, Zádori D (2020) Cerebellar predominant increase in mRNA expression levels of Sirt1 and Sirt3 isoforms in a transgenic mouse model of Huntington's disease. Neurochem Res (accepted publication) (original paper; IF (2018): 2,782)

II. Salamon A, Török R, Sümegi E, Boros F, Pesei ZG, Fort Molnár M, Veres G, Zádori D, Vécsei L, Klivényi P (2019) The effect of physical stimuli on the expression level of key elements in mitochondrial biogenesis. Neurosci Lett 698: 13-8 (original paper; IF (2018): 2,173)

III. Török R, Salamon A, Sümegi E, Zádori D, Veres G, Fort Molnár M, Vécsei L, Klivényi P (2017) Effect of MPTP on mRNA expression of PGC-1 $\alpha$ in mouse brain. Brain Res 1660: 206 (original paper; IF: $\mathbf{3 , 1 2 5}$ )

TOTAL IMPACT FACTOR OF ORIGINAL PAPERS DIRECTLY RELATED TO THE THESIS: $\mathbf{8 , 0 8}$

\section{PUBLICATIONS NOT DIRECTLY RELATED TO THE THESIS}

I. Salamon A, Dézsi L, Radics B, Varga ET, Hortobágyi T, Tömösvári A, Vécsei L, Klivényi P, Rajda C (2020) CANOMAD syndrome with respiratory failure. Ideggyogy Sz 73: 141-4 (case report; IF (2018): 0,113)

II. Szpisjak L, Salamon A, Zádori D, Klivényi P, Vécsei L (2020) Selecting dopamine depleters for hyperkinetic movement disorders: how do we choose? Expert Opin Pharmacother 21: 1-4 (editorial article; IF (2019): 0) 
III. Salamon A, Zádori D, Szpisjak L, Klivényi P, Vécsei L (2019) Neuroprotection in Parkinson's disease: facts and hopes. J Neural Transm (Vienna) 127: 821-9 (review article; IF (2018): 2,903)

IV. Salamon A, Zádori D, Szpisjak L, Klivényi P, Vécsei L (2019) Opicapone for the treatment of Parkinson's disease: an update. Expert Opin Pharmacother 20: 2201-7 (review article; IF (2018): 3,038)

V. Salamon A, Zádori D, Horváth E, Vécsei L, Klivényi P (2019) Zonisamide treatment in myoclonus-dystonia. Orv Hetil 160: 1353-7 (case report; IF (2018): 0,564)

VI. Salamon A, Faragó P, Németh VL, Szépfalusi N, Horváth E, Vass A, Bereczky Z, Tajti J, Vécsei L, Klivényi P, Zádori D (2019) Multiple ischemic stroke in Osler-Rendu-Weber disease. Ideggyogy Sz 72: 65-70 (case report; IF (2018): 0,113)

TOTAL IMPACT FACTOR OF PAPERS NOT DIRECTLY RELATED TO THE THESIS: 6,731 .

CUMULATIVE IMPACT FACTOR: 14,81 


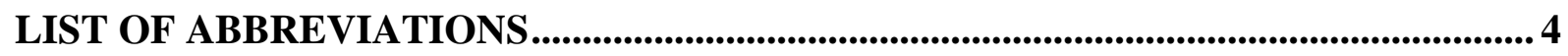

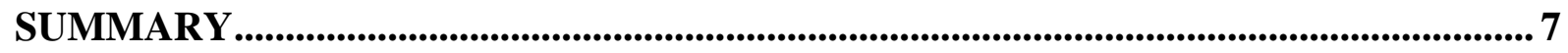

I - INTRODUCTION …........................................................................................................ 8

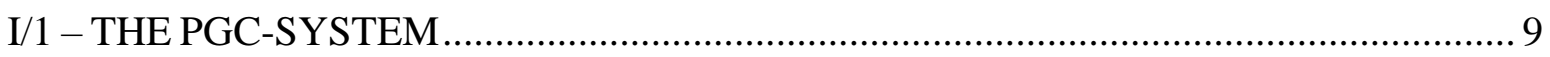

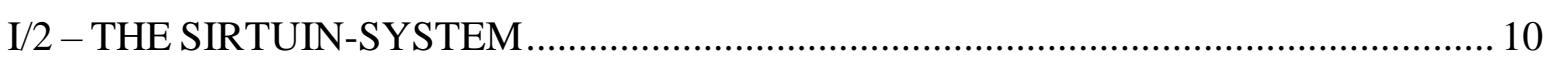

I/3 - INTERACTION BETWEEN THE PGC- AND SIRTUIN-SYSTEMS ....................... 12

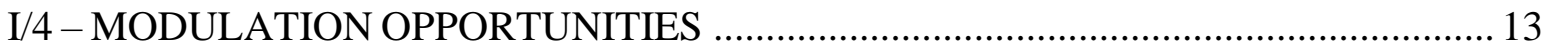

I/5 - ROLE OF PGC- AND SIRTUIN-SYSTEMS IN SOME MODELS OF

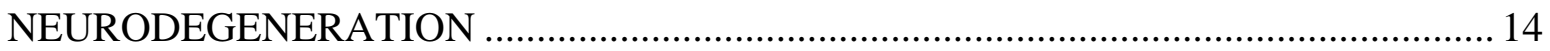

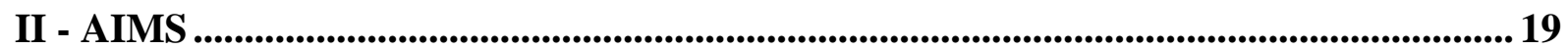

III - MATERIALS AND METHODS............................................................................. 20

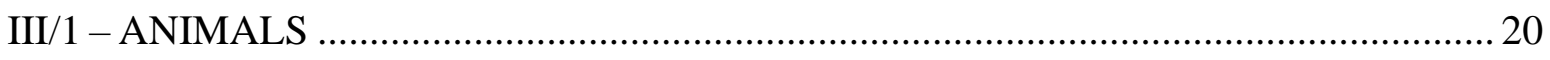

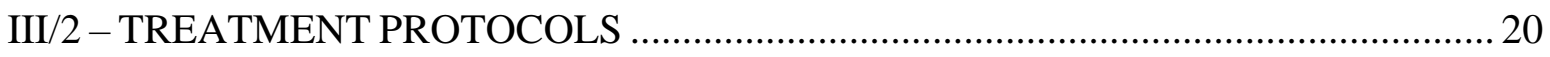

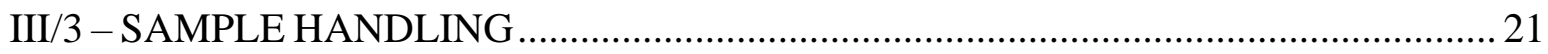

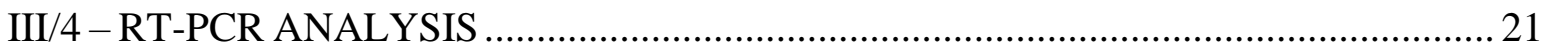

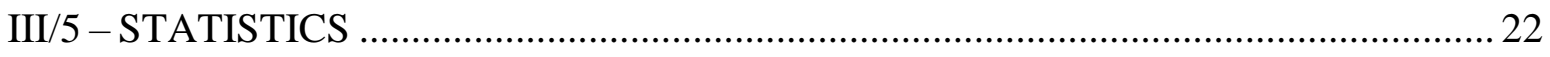

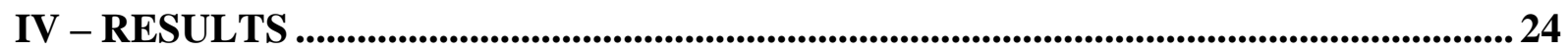

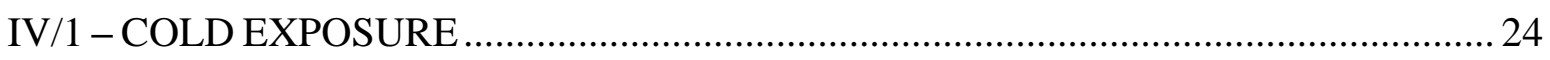

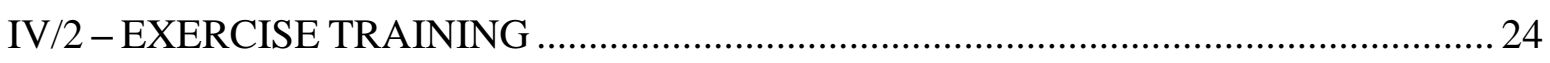

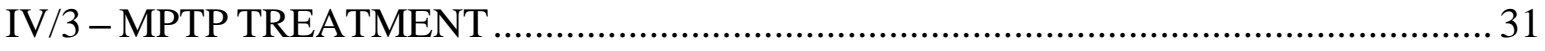

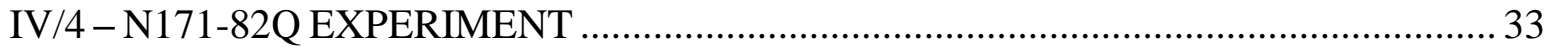

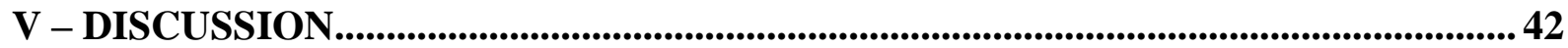

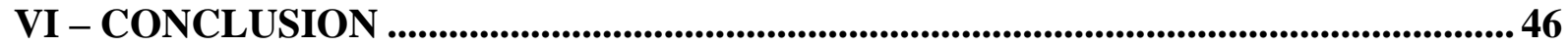

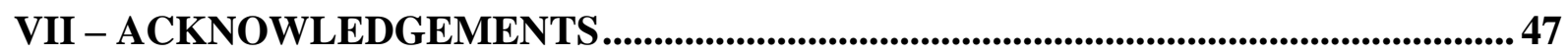

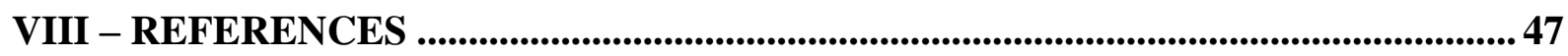




\section{LIST OF ABBREVIATIONS}

AAV

AceCS2

$\mathrm{AD}$

ALS

AP

APC

$\mathrm{A} \beta$

BAT

BP

BSKO

CNS-Pgc-1 $\alpha$

CNS-PGC-1 $\alpha$

CPS1

CR

CREB

ctrl

DR

ERR

ex

EX

FL-Pgc-1 $\alpha$

FL-PGC-1 $\alpha$

FOXO

FTD

G6PD

GDH

GPx

$\mathrm{H}$

HD

HIF adeno-associated virus

acetyl-CoA synthetase 2

Alzheimer's disease

amyotrophic lateral sclerosis

alternative promoter

adenomatous polyposis coli protein

amyloid beta protein

brown adipose tissue

brain (CNS)-specific promoter

brain-specific Sirt1 knockout mice

central nervous system (brain)-specific promoter (mRNA)

central nervous system (brain)-specific promoter (protein)

carbamoyl phosphate synthetase 1

calorie restriction

cAMP response element-binding protein (CREB)

control

dietary restriction

estrogen-related receptor

exon

exposed

full-length PGC-1 $\alpha$ (mRNA)

full-length PGC-1 $\alpha$ (protein)

forkhead box $\mathrm{O}$

frontotemporal dementia

glucose-6-phosphate dehydrogenase

glutamate dehydrogenase

glutathione peroxidase

histone

Huntington's disease

hypoxia-inducible factor 
HMGCS2

HNF4 $\alpha$

Hsp70

$H t t$

i.p.

IDH

IT15

$\mathrm{KO}$

K-RAS

LCAD

LP

MAO-B

MEF2C

$m H t t$

MPTP

$\mathrm{NF}-\kappa \mathrm{B}$

NRF

NT-Pgc-1 $\alpha$

NT-PGC-1 $\alpha$

PBS

PD

$P g c-1 \alpha$

Pgc- $1 \alpha$

PGC-1 $\alpha$

Pgc- $1 \beta$

PGC-1 $\beta$

Pitx3

PP
3-hydroxy-3-methylglutaryl-CoA synthase 2

hepatocyte nuclear factor

heat shock protein 70

huntingtin protein

intraperitoneally

isocitrate dehydrogenase

interesting transcript 15

knockout

Kirsten rat sarcoma 2 viral oncogene homolog protein

long chain acyl CoA dehydrogenase

liver-specific promoter

monoamine oxidase B

myocyte-specific enhancer factor $2 \mathrm{C}$

mutant huntingtin protein

1-methyl-4-phenyl-1,2,3,6-tetrahydropyridine

nuclear factor $\kappa \mathrm{B}$

nuclear respiratory factor

$\mathrm{N}$-truncated PGC-1 $\alpha$ isoform (mRNA)

$\mathrm{N}$-truncated PGC-1 $\alpha$ isoform (protein)

phosphate-buffered saline

Parkinson's disease

Pgc-1 $\alpha$ gene

peroxisome proliferator-activated receptor- $\gamma$ coactivator $1 \alpha$ (mRNA)

peroxisome proliferator-activated receptor- $\gamma$ coactivator $1 \alpha$ (protein)

peroxisome proliferator-activated receptor- $\gamma$ coactivator $1 \beta$ (mRNA)

peroxisome proliferator-activated receptor- $\gamma$ coactivator $1 \beta$ (protein)

pituitary homeobox 3 protein

proximal promoter 
PPAR

PRC

RAR

REF-Pgc- $1 \alpha$

REF-PGC-1 $\alpha$

RESV

RNA Pol I

ROI

RPM

RT-PCR

$\mathrm{SDH}$

Sir2

SIRT

Sirt1-FL(/Sirt1-Fl)

SIRT1-FL (/SIRT1-Fl)

Sirt1- $\Delta 8$

SIRT1- $\Delta 8$

Sirt1- $\Delta E 2$

SIRT1- $\Delta$ E2

Sirt3-M1, -2, -3

SIRT3-M1, -2, -3

SN

SOD

TAF4

$\operatorname{tg}$

$\mathrm{TH}$

TNF $\alpha$

TR

UCP

VLCAD

WAT

wt peroxisome proliferator-activated receptor

PGC-related coactivator

retinoic acid receptor

reference PGC-1 $\alpha$ promoter (mRNA)

reference PGC-1 $\alpha$ promoter (protein)

resveratrol

RNA polymerase I

reactive oxygen intermediate

revolutions per minute

real-time polymerase chain reaction

succinate dehydrogenase

silent information regulator 2

homolog of the silent information regulator 2

Full-length Sirt1 isoforms (mRNA)

Full-length Sirt1 isoform (protein)

SIRT1 isoform (lack of exon 8) (mRNA)

SIRT1 isoform (lack of exon 8) (protein)

SIRT1 isoform (lack of exon 2) (mRNA)

SIRT1 isoform (lack of exon 2) (protein)

SIRT3 transcript isoforms (mRNA)

SIRT3 transcript isoforms (protein)

substantia nigra

superoxide dismutase

transcription initiation factor TFIID subunit 4

transgene

tyrosine hydroxylase

tumor necrosis factor-alpha

thyroid hormone receptor

uncoupling protein

very long chain acyl CoA dehydrogenase

white adipose tissue

wild-type 


\section{SUMMARY}

Neurodegenerative diseases are becoming an increasingly serious health care problem in developed countries as the life expectancy increases, while the pathomechanism of the neurodegeneration is not fully understood yet. It seems that mitochondrial dysfunction might play a crucial role in the development of this process. The PGC- and the Sirtuin molecular families can induce the mitochondrial biogenesis and to interact with the neurodegenerative mechanism. Currently there is only a limited amount of data focusing on the brain region specific alteration of the isoforms of these systems. Accordingly, the aim of these experiments was to determine the mRNA level of some important elements of the PGC- and Sirtuin-systems (FL-, NT-, CNS-, REF-Pgc-1 $\alpha$, Sirt1-Fl, Sirt3-M1/M2/M3) in three brain regions involved in the regulation of motor functions (striatum, cortex, cerebellum) after different stimuli. Following two cold exposure $\left(4^{\circ} \mathrm{C}\right)$ protocols $(200$ and $900 \mathrm{~min})$ we found no detectable changes of Pgc-1 $\alpha$ transcripts in different brain areas. In contrast, after short cold exposure the cortical Sirt-M1 increased and the cerebellar Sirt3-M3 decreased. Longer cold exposure resulted in a relative decrease in the cortical Sirt1-Fl and striatal Sirt3-M1 levels. The effect of training was also tested in two different protocols. After 5 days training (short-term) no detectable change was identified in the PGC-system. From the perspective of Sirtuins, we found a cortical Sirt1-Fl mRNA elevation, but the other isoforms remained stable. Longer training period (12 days) resulted in a significant cerebellar activation in both molecular families (FL-, NT-, CNS-, REF-Pgc-1 $\alpha$, Sirt3-M1/M2). To test further the systems' behavior, we used the widely accepted MPTP toxin model of Parkinson's disease. The animals were treated with intraperitoneally administered MPTP (5x1 inj./day) and were dissected 90 min or 1 week after the last injection. In the PGC-system we identified a transient elevation in all tested brain regions. On the other hand, the effect of MPTP to the tested elements of Sirtuin-system was negligible. A transgenic animal model of Huntington's disease (N171-82Q) was also applied in the set of experiments. We found that in case of the striatum and cortex, the presence of the transgene resulted in a significant increase in Sirt3-M3 and Sirt1-Fl mRNA levels, respectively, whereas in case of the cerebellum the transgene resulted in increased expression of all the assessed subtypes and isoforms. In conclusion, we presume that our cooling protocol was ineffective to reduce the core temperature appropriately. In contrast, long-term training protocol 
was able to induce prominently the cerebellar PGC- and Sirtuin-systems, which emphasize the importance of cerebellum from the view of neuroprotection. Furthermore, we conclude that MPTP induces the PGC-system not only in the striatum and cortex, but also in the cerebellum. The effect of MPTP on Sirtuin-systems seems to be slight. In the N171-82Q transgenic HD model the unequivocal cerebellar Sirtuin activation with presumed compensatory role suggests that the cerebellum might be another key player in HD in addition to the most severely affected striatum.

\section{I - INTRODUCTION}

Chronic diseases are becoming increasingly serious health care problems in developed countries as the life expectancy increases (Hajat and Stein, 2018). In addition to the most common chronic cardiovascular diseases and cancers, neurodegenerative diseases are also having a significant influence on the health financing system (Sambamoorthi et al., 2015). Alzheimer's disease (AD) (prevalence (USA): 1200 per 100,000), Parkinson's disease (PD) (prevalence (USA): 300 per 100,000), frontotemporal dementia (FTD) (prevalence (USA): 14 per 100,000), amyotrophic lateral sclerosis (ALS) (prevalence (USA): 7 per 100,000) and Huntington's disease (HD) (prevalence (Caucasian population): 5 per 100,000) are the relevant elements of the heterogeneous group of neurodegenerative disorders (Rawlins et al., 2016; Relja, 2004). To date, several common clinical (e.g. the presence of Parkinsonian symptoms) and molecular similarities (e.g. accumulations of special type of proteins; selective neuronal damage) have been identified amongst these neurological conditions (Ahmad et al., 2017; Snowden et al., 1995). However, it seems that mitochondria may be one of the most important common subcellular hotspots behind the different conditions (Lezi and Swerdlow, 2012). For this reason, significant research activity has been directed to the characterization of mitochondrial dysfunction and the identification of potential neuroprotective molecular targets (Arun et al., 2016; Bose and Beal, 2016; Cabezas-Opazo et al., 2015; Costa and Scorrano, 2012; Shi et al., 2010). During the above-mentioned process, amongst others two interdependent metabolic master regulator families have been identified, namely the peroxisome proliferatoractivated receptor- $\gamma$ coactivator (PGC)- and the silent information regulator 2 homologues (Sirtuin)-family (Jęśko et al., 2017; Johri et al., 2013). 


\section{I/1 - THE PGC-SYSTEM}

There are three members of the PGC-family: (1) peroxisome proliferator-activated receptor- $\gamma$ coactivator $1 \alpha$ (PGC-1 $\alpha)$, (2) peroxisome proliferator-activated receptor- $\gamma$ coactivator $1 \beta$ (PGC-1 $\beta$ ) and (3) PGC-related coactivator (PRC) (Austin and St-Pierre, 2012). PGC-1 $\alpha$ is the most widely tested from the perspective of neurodegeneration. PGC-1 $\alpha$ was identified around 20 years ago as a cold-inducible coactivator of adaptive thermogenesis in

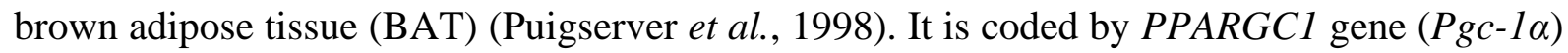
(13 exons; located on the $5^{\text {th }}$ chromosome (mice)) (Esterbauer et al., 1999; Liang and Ward, 2006). This master regulator participates in the gluconeogenesis of liver besides adaptive thermogenesis (BAT) and furthermore, in the fiber-type switching of skeletal muscle (type II to type I) as well and stimulates mitochondrial biogenesis and fatty acid $\beta$-oxidation (Puigserver et Spiegelman, 2003). The most important molecular targets are inter alia the followings: uncoupling proteins (UCPs), peroxisome proliferator-activated receptors (PPARs), reactive oxygen intermediate (ROI), defense enzymes (superoxide dismutases (SODs) and glutathione peroxidase (GPx)), thyroid hormone receptors (TRs), retinoic acid receptor (RAR), nuclear respiratory factors (NRFs), estrogen-related receptors (ERRs), myocyte-specific enhancer factor 2C (MEF2C), hepatocyte nuclear factor $4 \alpha(\mathrm{HNF} 4 \alpha)$ and forkhead box Os (FOXOs) (Lin et al., 2002, 2005; McGill and Beal, 2006; Puigserver et al., 1998; Puigserver and Spiegelman, 2003; St-Pierre et al., 2006; Zhang et al., 2009). The complexity of the PGC-1 $\alpha$-system is further enhanced by the fact that more than 10 isoforms are currently known, resulting from alternative splicing and promoter usage (Martínez-Redondo et al., 2015; Johri et al., 2011). These molecular processes are often coupled. The two main groups of the splicing variants are the full-length (FL-Pgc-1 $\alpha(1 /-\mathrm{a}) ; 797$ amino acid (AA)) and the N-terminal truncated (NT-Pgc$1 \alpha(-a) ; 270$ AA) isoforms (Zhang et al., 2009). The shorter, but also active NT-Pgc-1 $\alpha$ isoform is resulted from the insertion of an in-frame stop codon (31 base pair (bp)) between exon 6 and 7 (Zhang et al., 2009). From the perspective of the promoters, in addition to the canonical proximal (or reference) (PP; REF-Pgc-1 $\alpha$ ) and alternative promoters (AP), tissue-specific promoters have also been described (brain-specific (BP; CNS-Pgc-1 $\alpha$ ) and liver-specific promoters (LP)) (Martínez-Redondo et al., 2015; Soyal et al., 2012). Some research data suggest that different environmental or pharmacological stimuli result in a tissue-specific 
expression pattern of the above-mentioned isoforms (Lochmann et al., 2015; Wen et al., 2014). Currently there is only a limited amount of data about the isoform specific distribution of these in the brain. In the murine brain total Pgc-1 $\alpha$ was detected in the cortex, striatum, globus pallidus, substantia nigra and hippocampus (Tritos et al., 2003). Interestingly in the mouse brain there is a relative dominance of NT-Pgc-1 $\alpha$ isoform (Zhang et al., 2009). Additionally, mitochondrial density is increased in cortical, midbrain and cerebellar Pgc-1 $\alpha$ overexpressing neuronal cells (Wareski et al., 2009). Pgc-1 $\alpha$ was widely tested in the context of the neurodegenerative disorders. In the models of $P g c-1 \alpha$ deficiency resulted in the acceleration of the neurodegenerative mechanism, however, the stimulation of gene expression slowed it down (Róna-Vörös and Weydt, 2010). The $P g c-1 \alpha$ deficient mice developed HD-like phenomenon (hyperactivity, limb clasping and impaired thermoregulation), and furthermore, a spongiform striatal degeneration was observed in the brain of these animals (Lin et al., 2004; Leone et al., 2005). It is hypothesized that the mutant huntingtin ( $m H t t)$ interferes with the expression of $P g c-1 \alpha$ via binding to the promoter with cyclic adenosine monophosphate (cAMP) response element-binding protein (CREB) / transcription initiation factor TFIID subunit 4 (TAF4) complex (transcriptional repression) (Cui et al., 2006). In the MPTP model of Parkinson's disease, the $P g c-1 \alpha$ deficient mice are more vulnerable to the toxin (St-Pierre et al., 2006). On the other hand, the pharmacological (resveratrol (RESV)) or genetical overexpression of $P g c$ $1 \alpha$ protects the dopaminergic neurons against toxin-induced neurodegeneration (Mudò et al., 2012).

\section{I/2 - THE SIRTUIN-SYSTEM}

Sirtuins are mainly $\mathrm{NAD}^{+}$-dependent deacetylases, which react with the acetyllysine residues of various regulated proteins (Cen et al., 2011). The molecularly highly conserved Sirtuins were first identified in Saccharomyces cerevisiae (Silent information regulator 2 (Sir2)) (Paraíso et al., 2013; Zakhary et al., 2010). One of the most studied properties of Sirtuins are their lifelong increasing characteristic, however, the results in different species are controversial (Blander and Guarente, 2004; Cen et al., 2011). The effect of calorie restriction (CR) on Sirtuinsystem is widely studied. It was showed that CR lead (through the modification of $\mathrm{NAD}^{+} / \mathrm{NADH}$ ratio) to the activation of some Sirtuin subtypes (Qui et al., 2010). Currently 
seven Sirtuin subtypes (Sirt1-7) have been identified (Anekonda and Reddy, 2006; Cen et al., 2011; Donmez, 2012; Kelly, 2010a, b; Nunomura et al., 2007). These subtypes show different subcellular localization (Cen et al., 2011). Sirt1, -6 and -7 are mostly localized in the nucleus, Sirt3, -4, -5 in the mitochondria, while Sirt2 in the cytosol (Cen et al., 2011; Michishita et al., 2005). Certain Sirtuins have additional enzymatic properties besides deacetylase function: SIRT2 - demyristoylation, SIRT4 and -6 - ADP-ribosylation, SIRT5 - demalonylation, desuccinylation (Paraíso et al., 2013; Brenmoehl and Hoeflich, 2013). Like Pgc-1 $\alpha$, Sirtuins are responsible for regulating many proteins too. The main targets of the different Sirtuin subtypes are the followings: SIRT1 - histones (H1, -3, -4), tumor protein p53 (p53), nuclear factor- $\kappa \mathrm{B}(\mathrm{NF}-\kappa \mathrm{B})$, FOXOs, hypoxia-inducible factors (HIFs); SIRT2 - H3, -4, $\alpha$-tubulin, FOXOs, p53, NF-kB, HIFs, $\beta$-secretase, adenomatous polyposis coli protein (APC), Kirsten rat sarcoma 2 viral oncogene homolog protein (K-RAS); SIRT3 - H4, SODs, acetyl-CoA synthetase 2 (AceCS2), succinate dehydrogenase (SDH), 3-hydroxy-3-methylglutaryl-CoA synthase 2 (HMGCS2), (very) long chain acyl CoA dehydrogenase ((V)LCAD); SIRT4 glutamate dehydrogenase (GDH), heat shock protein 70 (Hsp70); SIRT5 - carbamoyl phosphate synthetase 1 (CPS1), HMGCS2, SOD1, VLCAD, cytochrome c, isocitrate dehydrogenase (IDH), glucose-6-phosphate dehydrogenase (G6PD); SIRT6 - H3, tumor necrosis factor-alpha (TNF $\alpha$ ); SIRT7 - H3, RNA polymerase I (RNA Pol I), p53 (Hallows et al., 2006; Schiedel et al., 2018). From the molecular targets detailed above, it becomes evident that Sirtuins play a very important role in the regulation of genomic stability, tumor suppression and cellular energetic processes. Furthermore, the isoforms of Sirt1 and Sirt3 make the Sirtuinsystem much more complex. These isoforms are originated from the alternative splicing and promoter usage similar to that of the PGC-system. Besides the full-length form of Sirt1 (Sirt1$\mathrm{Fl}$ ), two additional isoforms can be produced with specially designed PCR primers: Sirt1- $\Delta 8$ (lack of exon 8) and Sirt- $\Delta \mathrm{E} 2$ (lack of exon 2)). However, the biological role of these isoforms is questionable despite the dominant expression in the brain (Deota et al., 2017; Lynch et al., 2010). Sirt3 has also three transcript variants (Sirt3-M1 (AA (1-334)), -M2 (AA (15-334)) and -M3 (AA (88-334)), which produce three protein subtypes with various length of the N-terminal region (Bao et al., 2010; Cooper et al., 2009; Jin et al., 2009; Yang et al., 2010). Experimental data showed that Sirt3-M1 and -M2 splice variants are mainly localized in the mitochondria, while -M3 in the nucleus (Nogueiras et al., 2012). Limited data (mostly murine models) are available for the brain distribution of each subtypes. SIRT1 is normally localized in the neuronal and glial cells of cerebellum, cortex, hippocampus and hypothalamus (Hadem et al., 2019; Kelly, 2010b). SIRT2 has a prominent expression in the neurons and glias (especially in the 
oligodendroglias and astrocytes) (cerebellum, spinal cord, striatum, hippocampus) (Gomes et al., 2015; Kelly, 2010b; Maxwell et al., 2011; She et al., 2017). SIRT3 shows a pronounced species dependence, however, it seems to be mostly localized in the cortical neurons (Jęśko et al., 2017; She et al., 2017). SIRT4 is localized also in the cortex and in the hippocampus (She et al., 2017). SIRT5 and -7 can be found in the frontal lobe neurons (She et al., 2017). The effect of SIRT1 on neurodegeneration has been extensively tested. In AD SIRT1 reduces the production of $A \beta$ peptide under oxidative stress in mouse hippocampus, and furthermore, it stimulates the autophagy to help removing the pathological proteins (Sun et al., 2010; Zhang et al., 2011). It seems that the total SIRT1 and -3 protein levels correlate with the disease stage of AD (Jęśko et al., 2017). In a similar way, in PD, SIRT1 reduces the formation of $\alpha$-synuclein aggregates as well as promotes the autophagy which lead to the removal of the pathological proteins (Donmez et al., 2012; Lee et al., 2008; Zhang et al., 2011, 2012). In HD the pharmacological overexpression (RESV) of Sirtuins resulted in the improvement of the neuronal dysfunction in nematodes and mammalian neurons as well. In postmortem striatal and cortical brain samples of HD patients Sirt1 mRNA level was reduced (Baldo et al., 2019; Parker et al., 2005).

\section{I/3 - INTERACTION BETWEEN THE PGC- AND SIRTUIN-SYSTEMS}

Cumulated data suggest that the above detailed two important neuroprotective systems are not independent (Amat et al., 2009; Nemoto et al., 2005; Rodgers et al., 2005). In the cytoplasm there is an interaction between PGC-1 $\alpha$ and SIRT1 (SIRT1 connects to the central, regulatory region of PGC-1 $\alpha$ ) (Lin et al., 2005; Zhong and Mostoslavsky, 2011). During this molecular linkage, SIRT1 (some data suggest that SIRT3 as well) deacetylates PGC-1 $\alpha$, which leads to the activation of the downstream targets and the promotion of mitochondrial biogenesis, oxidative phosphorylation and energy production (Brenmoehl and Hoeflich, 2013; Kong et al., 2010; Nemoto et al., 2005; Rodgers et al., 2005). Furthermore, SIRT1 stimulates the promoter of $P g c-1 \alpha$ gene in special environmental context (fasting, calorie (dietary) restriction (CR (DR)) (Amat et al., 2009; Dominy et al., 2009; Kelly, 2010a; Nemoto et al., 2005; Rasouri et al., 2007). 


\section{I/4 - MODULATION OPPORTUNITIES}

The regulatory role of the PGC- and Sirtuin-systems in various metabolic pathways, as well as the potential neuroprotective effect led to several scientific efforts to modulate these molecules. These efforts can be divided into three main groups: (1) - environmental activation (cold exposure, training, CR (DR)), (2) - pharmacological activation (e.g. RESV) and (3) genetic manipulation (e.g. overexpression, knockout (KO) animals). In the following paragraphs, only modulation options relevant to the current researches are detailed.

The effect of the environmental temperature on the PGC- and Sirtuin-systems was tested in the brown- and white adipose tissue (BAT, WAT), and in the skeletal muscle as well. However, there is only limited data available about the effect on the brain. In the BAT, cold exposure $\left(4^{\circ} \mathrm{C}\right.$ for $\left.5 \mathrm{~h}\right)$ led to a significant increase in both FL- and NT-Pgc-1 $\alpha$ isoforms (6week-old C57BL/6J male mice) (Zhang et al., 2009). Sirt1 and -3 mRNA levels in BAT also showed continuous significant elevations to cold exposure $\left(5^{\circ} \mathrm{C}\right.$ for $\left.0-12 \mathrm{~h}\right)$ and decreases if the room temperature was elevated $\left(27.5^{\circ} \mathrm{C}\right.$ for $\left.16 \mathrm{~h}\right)(8$-week-old C57BL/6 male mice) (Jokinen et al., 2017; Shi et al., 2005). A longer cooling period (1-3 days, $\left.4^{\circ} \mathrm{C}\right)$ was able to induce (the peak value was on day 1) the Pgc-1 $\alpha$ expression in retroperitoneal WAT of 3-month-old male rats as well (Jankovic et al., 2015). On $5^{\circ} \mathrm{C}$, Sirt2/SIRT2 (mRNA and protein) were also inducible in BAT after 6 hours of cooling (4-week-old C57BL/6 male mice) (Wang and Tong, 2009). 3 hours after the exercise (30 min), in the skeletal muscle of 9 physically active men (mean age: 25.8 years) the cooling of the vastus medialis $\left(10^{\circ} \mathrm{C}\right)$ resulted in the elevation of the Pgc-1 $\alpha$ level (Ihsan et al., 2014). Consistent with these data, in the skeletal muscle of 4-monthold male rats a significant elevation of the PGC-1 $\alpha$ protein level (from day 3 to day 45) was detectable as well (Stancic et al., 2013). There was a significant increase after 6 hours of cooling on $4^{\circ} \mathrm{C}$ in the relative mRNA level of Pgc-1 $\alpha$ in the skeletal muscle of Sirt1 overexpressing transgenic (tg) mice (Gerhart-Hines et al., 2011). Regarding the central nervous system (CNS), it is known that HD tg animal models (R6/2 and N171-82Q) are susceptible to hypothermia (Weydt et al., 2006). Trios et al. (2003) could not detect any Pgc-1 $\alpha$ level alteration in the brain of adult (18-20-week-old) male C57B1/6J mice after 4 hours $\left(4^{\circ} \mathrm{C}\right)$ of cooling.

The impact of exercise on PGC- and Sirtuin-systems was widely tested, however, the diversity of the models and the training strategies make the interpretation and comparison of these results complicated. It is proved that training elevates the level of PGC- $1 \alpha$ and SIRT1 in 
the skeletal muscle (interestingly the intensity of the training influences the promoter usage in the muscle) (Costa et al., 2010; Huang et al., 2015; Lochmann et al., 2015; Wen et al., 2014). Steiner et al. (2011) found that after 8 weeks of treadmill training Pgc-1 $\alpha$ and Sirt1 mRNA level elevations were detectable in different brain regions of ICR (Institute of Cancer Research) mice (8-week-old) (Pgc-1 $\alpha$ - musculus soleus, brainstem, cortex, frontal lobe, hippocampus, hypothalamus, midbrain; Sirt1 - cortex, frontal lobe, hippocampus, hypothalamus, midbrain). On the other hand, Lezi et al. (2013) found no alteration of Pgc-1 $\alpha$ and Sirt1 mRNA levels in the whole brain samples of C57BL/6 mice (4-month-old) after 6 weeks of moderate intensity treadmill training. Lezi et al. (2014) in their next study applied a longer training period (8 weeks), but there was no detectable Pgc-1 $\alpha$ mRNA alteration in the whole brain samples of the mice (C57BL/6; 18-month-old), whereas the nuclear PGC- $\alpha$ protein level significantly increased. The potential influencing role of the age of animals has arisen behind the incongruent data, and therefore, Gusdon et al. (2017) tried to clarify the question with a 17-day-long treadmill (with increasing intensity) protocol, where 2 representative age groups of C57BL/6 mice (only the striatum and cortex was processed) were used (4-week-old and 24-month-old). They found no alteration in the protein level either in the PGC-1 $\alpha$, or in the SIRT3 group. In young (5-week-old) rats (Sprague-Dawley) Bayod et al. (2011) found a cortical PGC-1 $\alpha$ and SIRT1 and a hippocampal SIRT1 protein elevation after 36 weeks of training. Belvirani and Okudan (2018) found that in young (3-month-old) Wistar rats the Pgc-1 $\alpha$ mRNA level was elevated in the hippocampus (90 days, free wheel running exercise). Nevertheless, this effect was no more detectable in older animals (20-month-old). In contrast, after 2 weeks of moderate intensity treadmill training there was a detectable elevation of PGC-1 $\alpha$ protein level in the hippocampus of 12-month-old Wistar rats (Marosi et al., 2012).

\section{I/5 - ROLE OF PGC- AND SIRTUIN-SYSTEMS IN SOME MODELS OF NEURODEGENERATION}

Parkinson's disease (PD) is clinically characterized by bradykinesia, tremor and/or muscle rigidity (Postuma et al., 2015). Behind this clinical constellation there is the cumulation of several complex pathological molecular processes which result in the loss of dopaminergic neurons in the substantia nigra (SN) (Allain et al., 2008; Mandel et al., 2003). At the time of 
diagnosis, around 30\% of dopaminergic neurons have already died (Cheng et al., 2010). The most important contributors of neurodegeneration are the followings: (1) MAO-B activity, (2) oxidative stress and reduced endogenous antioxidant capacity, (3) elevated iron level, (4) glutamatergic excitotoxicity, (5) abnormal protein aggregation, misfolding, (6) reduced level of trophic factors, (7) altered ion homeostasis and (8) neuroinflammation (Hirsch et al., 2013; Koutsilieri and Riederer 2007; Majláth et al., 2016; Mandel et al., 2003; Salamon et al., 2019; Zádori et al., 2011, 2012, 2013). One of the most widely known model of PD is the 1-methyl4-phenyl-1,2,3,6-tetrahydropyridine (MPTP) toxin (mitochondrial complex I inhibitor) treatment (Langston, 2017). Previously performed animal experiments hypothesized a potential neuroprotective effect of PGC-1 $\alpha$ against MPTP-induced neuronal cell damage (Mudò et al., 2012). In Pgc-1 $\alpha$ deficient animals the MPTP-induced damage was more robust, and the oxidative damage was more pronounced compared to wild-type (wt) controls (St-Pierre et al., 2006). However, the overexpression or the pharmacological activation (e.g. RESV) showed a potentially neuroprotective elevation after MPTP treatment (Breidert et al., 2002; Dehmer et al., 2004; Mudò et al., 2012). In contrast, Clark et al. (2012) found that the number of the tyrosine hydroxylase (TH) positive cells was unexpectedly reduced after 5 days of the last MPTP injection when unilateral SN $P g c-1 \alpha$ induction (adeno-associated virus (AAV), C57BL/6CR mice) was applied. This effect was associated with the loss of a transcription factor, namely pituitary homeobox 3 protein (Pitx3). Wang et al. (2019) also tested the effect of stereotactic injection of lentivirus (Pgc-1 $\alpha$ overexpression or silencing) in C57BL/6 mice. After the injection of lentivirus the mice were treated for 2 weeks with MPTP. They found an increase in the number of the TH-expressing cells and an elevated mitochondrial density in SN of the Pgc-1 $\alpha$ overexpressing group. The optimal timing of the dissection after the last MPTP injection is critical to be able to compare the experimental data. If the dissection was performed after $24 \mathrm{~h}$ of the last MPTP injection, the level of PGC-1 $\alpha$ was elevated and after $72 \mathrm{~h}$ it was normalized (short-term compensatory reaction) (Swanson et al., 2013). Two studies have tested the potential neuroprotective effect of Sirtl overexpression against MPTP toxicity (Kakefuda et al., 2009; Kitao et al., 2005). Kakefuda used neuron-specific enolase-driven Sirtl tg mice. In the tg mice there was no detectable neuroprotective effect against the toxin compared to the wt mice. Interestingly the tg animals exhibited a memory deficit (Kakefuda et al., 2009). In line with these results Kitao et al. (2005) found that in the SN of Sirt1-transgenic (prion promoterdriven) mice the number of TH-positive neurons was comparable with that of wt littermates. In conclusion, it seems that the Sirtl overexpression did not alleviate the toxic effect of MPTP. Other members of the Sirtuin-system were also tested by Liu et al. (2015a, b). They have proved 
that in Sirt3 and Sirt5 null mice the MPTP-induced nigrostriatal damage becomes dramatically pronounced.

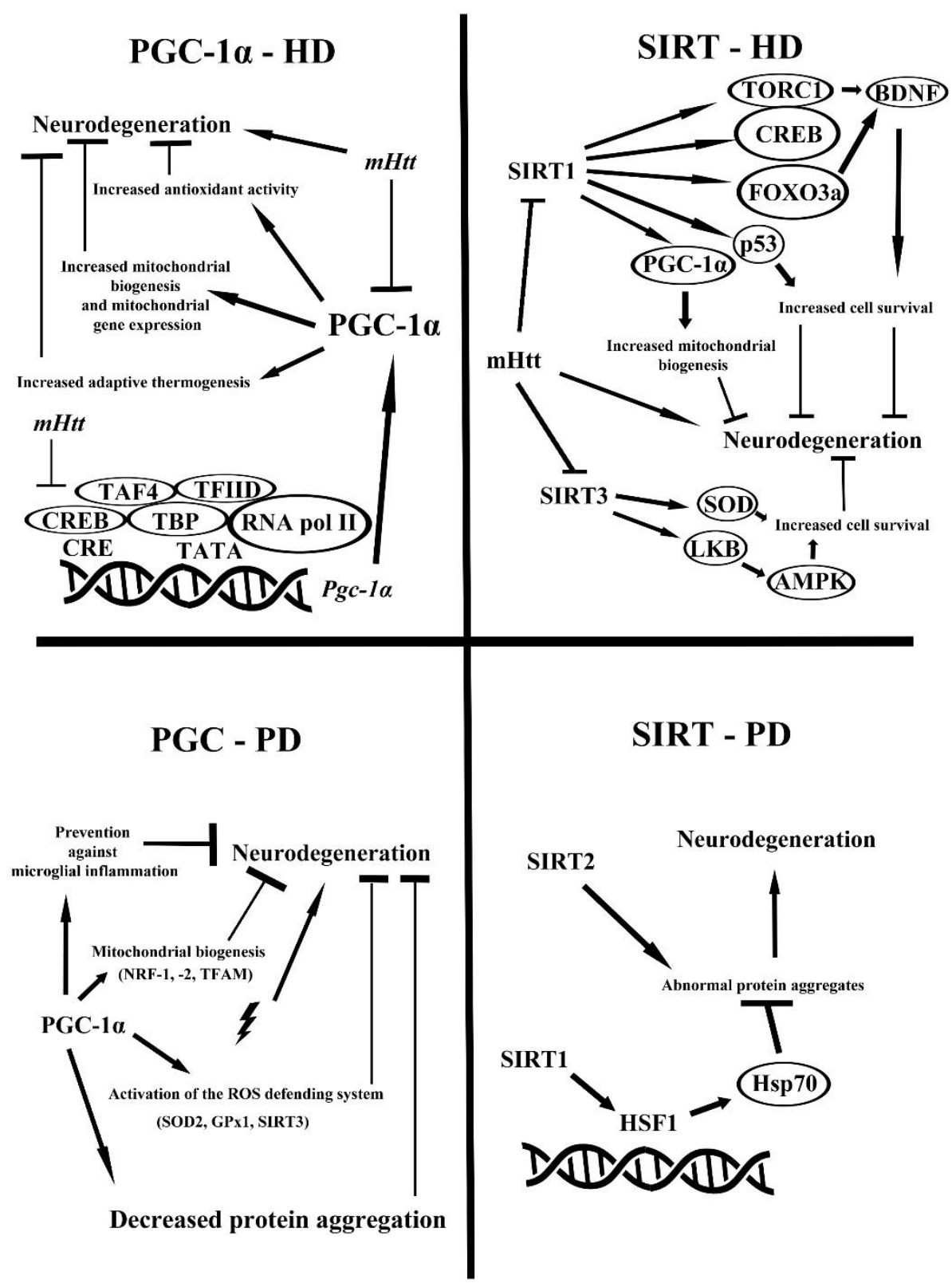

Figure 1 - Role of PGC- and Sirtuin-systems in Huntington's and Parkinson's diseases (Abbreviations: AMPK - 5' AMP-activated protein kinase; BDNF - Brain-derived neurotrophic factor; CRE - cAMP response element; CREB - cAMP response element-binding protein; FOXO3a - Forkhead box O3; GPx1 - Glutathione peroxidase 1; HD - Huntington's disease; HSF1 - Heat shock factor 1; Hsp70 - $70 \mathrm{kDa}$ heat shock protein; LKB - Tumor suppressor serine/threonine-protein kinase; $m H t t$ - mutant Huntingtin protein; NRF - Nuclear factor erythroid 2-related factor; p53 - TP53; PD - Parkinson's disease; RNA pol II - RNA polymerase type II; SOD - Superoxide dismutase; TAF4 - transcription initiation factor TFIID subunit 4; TATA TATA box; TBP - TATA-box binding protein; TFAM - Transcription factor A; TFIID Transcription factor II D; TORC1 - CREB-regulated transcription coactivator 1 (CRTC1)) (The following articles served as the basis for the figure: Herskovits and Guarente, 2013, 2014; RónaVörös and Weydt, 2010; McGill and Beal, 2006) 
Huntington's disease (HD) is an autosomal dominantly inherited neurodegenerative disease, which is caused by the expansion of CAG repeats in the IT15 gene encoding huntingtin protein (Htt) (Schulte and Littleton, 2011; Vonsattel et al., 1985). Previous works demonstrated that mutant huntingtin protein $(m H t t)$ inhibits the function of PGC-1 $\alpha$ via transcriptional dysregulation (Cui et al., 2006; Jodeiri Farshbaf and Ghaedi, 2017). The Pgc-1 $\alpha$ level was downregulated in the skeletal muscle samples of a transgenic HD mouse model and in human muscle biopsies as well, which refers to the potential role of the PGC-system in the muscle dysfunction in HD (Chaturvedi et al., 2009). From the perspective of the brain, striatal degeneration was demonstrated in transgenic models of HD (Weydt et al., 2006; Zádori et al., 2011). Furthermore, in the nucleus caudatus of premanifest HD patients the mRNA level of Pgc-1 $\alpha$ was significantly reduced (Cui et al., 2006). Kim et al. (2010) confirmed these results and found a relation between the stage of HD and the level of PGC-1 $\alpha$ protein in the nucleus caudatus (the immunoreactivity of PGC-1 $\alpha$ was gradually decreased (by $70 \%$ in Grade 4)). Interestingly, the lentiviral mediated overexpression of $P g c-1 \alpha$ in the striatum of R6/2 mice resulted in the improvement of the phenotype (Cui et al., 2006). Histological studies proved that the striatal medium spiny neurons are more vulnerable in HD, but the interneurons are relatively spared (Cui et al., 2006). Johri et al. (2011) were the first, who tested the potential influence of NT-Pgc-1 $\alpha$ in the pathogenesis of HD in transgenic murine models (R6/2, N17182Q, mouse Q111 striatal cells). They found a depletion of the N-truncated isoform in the Q111 striatal cells. On the other hand, the NT-Pgc-1 $\alpha$ level was upregulated in the striatal samples of older R6/2 and N171-82Q animals compared to the younger animals, which correlated with the human results. Török et al. (2015) was the first, who estimated the brain region specific distribution of FL- and NT-Pgc-1 $\alpha$ isoforms and tested the mRNA levels of REF- and CNSPgc-1 $\alpha$ promoters in 8-, 12- and 16-week-old N171-82Q mice. They found that the FL-Pgc-1 $\alpha$ mRNA level was significantly downregulated in the striatal and cortical samples of 8-week-old animals. The NT-Pgc-1 $\alpha$ level was, however, upregulated in the striatal and cortical samples of 16-week-old animals. Török et al. (2015) also tested a previously neglected (except the work of Steiner et al. (2011)) brain region, namely the cerebellum, where there was a strong significance of elevation regarding both isoforms. FL-Pgc- $1 \alpha$ mRNA level was increased in the brains of 12- and 16-week-old animals, and furthermore, the NT-Pgc-1 $\alpha$ level was elevated in all three age groups. They hypothesized that it is a compensatory phenomenon. The association between SIRTs and HD was also widely studied (Ajami et al., 2017; Jeong et al., 2011; Jiang et al., 2011; La Spada, 2012; Naia and Rego, 2015; Tabrizi et al., 2012). The published data about the Sirt1/SIRT1 mRNA and protein changes are somewhat inconsistent. In human brain 
tissue and in R6/1 transgenic model the SIRT1 protein level was reduced (Hathorn et al., 2011; Pallàs et al., 2008). In R6/2 (mean CAG repeats number (MRN): 204) and HdhQ150 (MRN: 165) the SIRT1 activity was reduced in the striatum and cerebellum (Tulino et al., 2016). The level of Sirt1/SIRT1 mRNA and protein were not affected by the presence of the transgene in the striatal samples of R6/2 mice. However, there was a decrease in the wt mice from 4 to 9 weeks (Tulino et al., 2016). The changes of the protein and mRNA levels of Sirt1/SIRT1 was opposite in the cerebellum of R6/2 mice (the Sirt 1 mRNA expression increased significantly by 9 and 14 weeks; the SIRT1 protein levels significantly decreased predominantly in wt mice by 14 weeks). In another model (HdhQ150) the SIRT1 protein level did not change (Tulino et al., 2016). Reynolds et al. (2018) reported later a whole-brain study (R6/2 mice (MRN: 144)), where the Sirt1 mRNA level elevated in the 5-, 8- and 11-week-old tg animals. In contrast to these findings, in the brain of 12-week-old animals (MRN: 182) the Sirt1 mRNA elevation was no more detectable. The protein level of SIRT1 was also consistently elevated in the brain of both 8- and 12-week-old animals (Reynolds et al., 2018). Jeong et al. (2011) crossed the brainspecific Sirt1 knockout mice (BSKO; genotype: Sirtlflox/flox) with the R6/2 HD model mice to test the potential neuroprotective effect of SIRT1 in HD. It resulted in the augmentation of the neuronal cell damage in the striatum (Jeong et al., 2011). In contrast, the overexpression of Sirt1 (Sirt1-KI-R6/2) led to a longer survival time and less dominant neuropathological alterations (Jeong et al., 2011). Jiang used other Sirt1-crossed models (N171-82Q and BAC HD), also demonstrating the deceleration of disease process (Jiang et al., 2011). The available data are limited regarding the role of other Sirtuin isoforms (SIRT2-7). However, it seems that SIRT2 might worsen the disease process in HD (Chopra et al., 2012; Maxwell et al., 2011). Some authors suggest that SIRT3 has an opposite effect (Ahn et al., 2008; Ajami et al., 2017; Fu et al., 2012; Neo and Tang, 2017). Fu et al. (2012) found that the striatal administration of a RESV dimer ( $\varepsilon$-viniferin) was able to reduce the ROS level through SIRT3-mediated superoxide dismutase 2 (SOD2) induction in striatal progenitor cells (Hdh(Q111)).

The data detailed above are seemingly incomplete. There is a lack of experimental data focusing on the role of the PGC- and Sirtuin-systems in different brain regions. Furthermore, increasing data suggest that isoforms resulting from alternative promoters and splicing usage may have an important biological role. We think that it is important to start the detailed characterization these systems in each neurodegenerative model, through which we will be able to identify novel therapeutic molecular targets. 


\section{II - AIMS}

\section{The aims of the work were:}

(1) To determine the effect of cold exposure $\left(4^{\circ} \mathrm{C} ; 200\right.$ or $\left.900 \mathrm{~min}\right)$ on the PGC- and Sirtuinsystems (FL-, NT-, CNS-, REF-Pgc-1 $\alpha$, Sirt1, Sirt3-M1/M2/M3) in different brain areas important in the regulation of motor functioning (striatum (Str), cortex (Ctx), cerebellum (Crb)) in $\mathrm{C} 57 \mathrm{Bl} / 6 \mathrm{~J}$ mice.

(2) To determine the effect of exercise training ( $2 \times 30 \mathrm{~min} /$ day; 5 or 12 days) on the PGCand Sirtuin-systems (FL-, NT-, CNS-, REF-Pgc-1 $\alpha$, Sirt1, Sirt3-M1/M2/M3) in different brain areas important in the regulation of motor functioning (Str, Ctx, Crb) in C57B1/6J mice.

(3) To determine the effect of MPTP (5x1 i.p. inj. (15 mg/kg) for 1 day; dissection 90 min or 1 week after the last injection) on the PGC- and Sirtuin-systems (FL-, NT-, CNS-, REF-Pgc-1 $\alpha$, Sirt1, Sirt3-M1/M2/M3) in different brain areas important for neurodegeneration (Str, $\mathrm{Ctx}, \mathrm{Crb})$ in $\mathrm{C} 57 \mathrm{~B} 1 / 6 \mathrm{~J}$ mice.

(4) To assess the mRNA expression pattern of Sirt1 and three isoforms of Sirt3 in the Str, Ctx and Crb using the N171-82Q tg mouse model of HD. Furthermore, to assess the effect related to the presence of the transgene and the possible effect of aging and gender. 


\title{
III - MATERIALS AND METHODS
}

\author{
III/1 - ANIMALS
}

In the cold exposure and exercise training experiments 20 -week-old female (C57Bl/6J) mice were involved. During the MPTP study 12-week-old male (C57B1/6J) mice were treated. To test the effect of transgene on the PGC- and Sirtuin-systems, 8-, 12- and 16-week-old N17182Q and their control (B6C3) wt mice with identical genetic background (female and male animals distributed equally) were used. All the tg animals were originally obtained from Jackson Laboratory (Bar Harbor, ME, USA). The mice were housed in cages under standard conditions with 12-12 h light-dark cycle and free access to food and water. The experiments were carried out in accordance with the European Communities Council Directive (86/609/EEC) and were approved by the local animal care committee (XI./846/2019, XXIV./352/2012).

\section{III/2 - TREATMENT PROTOCOLS}

COLD EXPOSURE - Animals were randomly divided into four groups $(\mathrm{n}=7-8$ in each group). The first group was kept at $4{ }^{\circ} \mathrm{C}$ for $40 \mathrm{~min} /$ day, for 5 days $(200 \mathrm{~min})$, the second one was kept $4^{\circ} \mathrm{C}$ for $180 \mathrm{~min} /$ day for 5 days $(900 \mathrm{~min}$ ). After the cold exposure, mice were placed back under standard conditions $\left(22-24^{\circ} \mathrm{C}\right)$. The third and fourth groups were control groups and were housed at $22-24^{\circ} \mathrm{C}$ in the same room.

EXERCISE TRAINING was performed using a rotarod. The mice were randomly allocated into four groups ( $n=5-8$ in each group). The first and second groups were the training groups. The mice were placed on the rotarod for a 2-session period (9.00 a.m., 4.00 p.m.) for 5 days (first group) or 12 days (second group) after a short learning period. The standard speed was 5 RPM for $30 \mathrm{~min}$. Prior to each training session, the mice were transported to the testing room for an acclimatization period of at least $30 \mathrm{~min}$. The third and fourth groups were control groups. 
MPTP TREATMENT - MPTP was dissolved in phosphate-buffered saline (PBS; pH adjusted to 7.4) and was administered intraperitoneally (i.p.). Animals were randomly divided into four groups ( $n=7-8$ in each group). The first and second groups received i.p. injection of $15 \mathrm{mg} / \mathrm{kg}$ body weight MPTP 5 times at $2 \mathrm{~h}$ intervals. The third and fourth groups served as the respective control groups and were injected with 0.1 M PBS according to the above-detailed treatment regimen.

\section{III/3 - SAMPLE HANDLING}

In the cold exposure and the exercise training experiments ninety minutes after the last session, the animals were deeply anesthetized with isoflurane (Forane; Abott Laboratories Hungary Ltd., Budapest, Hungary) and their brains were dissected immediately. In the MPTP experiments the animals in the first group were deeply anesthetized with isoflurane and the brains were dissected ninety minutes following the last MPTP injection (acute treatment - acute (day 1) assessment), while animals in the second group were deeply anesthetized and dissected only one week later (acute treatment - subacute (day 7) assessment). In the third and fourth (control) groups the dissection was made respective to the above-detailed acute and subacute groups. In the N171-82Q experiment, the animals were anesthetized and dissected when they reached the 8-, 12-, and 16 weeks of age (the control groups were followed this method as well). The method of dissection was the same for each experiment. During the process the brains were rapidly removed on ice and immediately halved at the midline and then both hemispheres were further cut to obtain the striatum, cortex and cerebellum. All the samples were stored at $-80^{\circ} \mathrm{C}$ until the real-time polymerase chain reaction (RT-PCR).

\section{III/4 - RT-PCR ANALYSIS}

Total RNA was isolated from striatum, cortex and cerebellum with Trizol reagent according to the manufacturer's protocol (Molecular Research Center, USA). The concentration of RNA was measured with a MaestroNano spectrophotometer, and the integrity of RNA was randomly tested by gel electrophoresis using $1 \%$ agarose gel. cDNA was generated from $1 \mu \mathrm{g}$ of total RNA with random hexamer primers and reverse transcriptase according to 
the Revert Aid First Strand cDNA Synthesis Kit protocol (Thermo Scientific, USA). cDNA was kept at $-20^{\circ} \mathrm{C}$ until further use. RT-PCR was performed with a CFX 96 Real-Time System (Bio-Rad, USA) to detect changes in mRNA expression, using various primer pairs at a final volume of $20 \mu \mathrm{l}$. The following Pgc-1 $\alpha$ and Sirtuin primers were used (Chang et al., 2012;

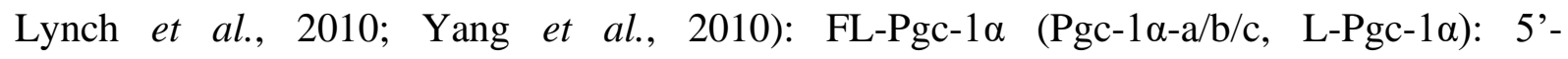
TGCCATTGTTAAGACCGAG-3' (forward; ex4/ex5) and 5'-TTGGGGTCATTTGGTGAC3' (reverse; ex6/ex7); NT-Pgc-1 $\alpha$ (NT-Pgc-1 $\alpha$-a/b/c): 5'-TGCCATTGTTAAGACCGAG-3' (forward; ex4/ex5) and 5'-GGTCACTGGAAGATATGGC-3' (reverse; ex6/7a (in-frame stop codon)); CNS-Pgc-1 $\alpha$ : 5'-AATTGGAGCCCCATGGATGAAGG-3' (forward; exB4) and 5'TCAAATGAGGGCAATCCGTC-3' (reverse; ex3); Ref-Pgc-1 $\alpha$ (Pgc-1 $\alpha$-a and NT-Pgc-1 $\alpha$-a): 5'-TGAGTCTGtatGGAGTGACATCGAGTG-3' (forward; ex1a/ex2) and 5'TCAAATGAGGGCAATCCGTC-3' (reverse; ex3); Sirt1: 5'GCACTAATTCCAAGTTCTATACCC-3' (forward; ex7/ex8) and 5'GTGGTACAGTTCTTTCAGGTG-3' (reverse; ex8); Sirt3-M1: 5'TCAGACTGTGGGGTCCGGGAGTGTTA-3' (forward; ex1b) and 5'CAACATGAAAAAGGGC-3' (reverse; ${ }^{2} \quad$ ex3); GACTGTGGGGTCCGGGAGGTGG-3' (forward; ex1b) and 5'-CAACATGAAAAAGGGC3' (reverse; ex3); Sirt3-M3: 5'-GGCGTTTGGCGAGGACTA-3' (forward; ex2) and 5'CAACATGAAAAAGGGC-3' (reverse; ex3). The thermal cyclic conditions were: Pgc-1 $\alpha$ primers: $95^{\circ} \mathrm{C}$ for $2 \mathrm{~min}$, followed by 40 cycles of $95^{\circ} \mathrm{C}$ for $10 \mathrm{~s}$, and $60^{\circ} \mathrm{C}$ for $30 \mathrm{~s}$. Sirt1: $95^{\circ} \mathrm{C}$ for $2 \mathrm{~min}$, followed by 40 cycles of $95^{\circ} \mathrm{C}$ for $10 \mathrm{~s}$, and $62^{\circ} \mathrm{C}$ for $30 \mathrm{~s}$; Sirt3-M1 and Sirt3-M2: $95^{\circ} \mathrm{C}$ for $2 \mathrm{~min}$, followed by 40 cycles of $95^{\circ} \mathrm{C}$ for $10 \mathrm{~s}$, and $62.4^{\circ} \mathrm{C}$ for $30 \mathrm{~s}$; Sirt3-M3: $95^{\circ} \mathrm{C}$ for $2 \mathrm{~min}$, followed by 40 cycles of $95^{\circ} \mathrm{C}$ for $10 \mathrm{~s}$, and $56.6^{\circ} \mathrm{C}$ for $30 \mathrm{~s}$. Target gene expression was normalized to the endogenous control gene 18S rRNA (Applied Biosystems, USA). Relative expression was calculated by the $2{ }^{-{ }^{\Delta \Delta}} \mathrm{Ct}$ method (Livak and Schmittgen, 2001).

\section{III/5 - STATISTICS}

All statistical calculations were performed with the use of the freely available R software ( $R$ Development Core Team). The distribution of data populations was checked with the ShapiroWilk test, and Levene test was also performed for the analysis of the homogeneity of variances. To assess the differences between Pgc-1 $\alpha$ - and Sirtuin gene expression levels in all brain areas 
relative to their respective control groups, approximative (10,000 random permutation) two sample Fisher-Pitman permutation test was applied in case of cold exposure and exercise training experiments. In the MPTP experiments two-sample t-tests via Monte-Carlo permutation (with 10,000 random permutations) were performed. In the HD experiment in several cases the data were diverged from Gaussian distribution and the variances were not equal. For that reason we applied the Scheirer-Ray-Hare test to determine the differences between the investigated factors and their interaction as well. Afterwards, we carried out permutation t-tests as post hoc analysis for pairwise comparison and type I errors from multiple comparisons were controlled with false discovery rate. As some of the possible comparisons would not have yielded meaningful information regarding the a priori decided presumptions, a maximum of 9 pairwise comparisons were implemented in case of each subtype or isoform analyzed by each brain region. We calculated the gene expression level of Pgc-transcripts in all brain areas relative to FL-Pgc-1 $\alpha$ and CNS-Pgc- $1 \alpha$ control striatum groups. For Sirtuins we compared Sirt1 expression levels to control striatal Sirt1-Fl, and all Sirt3 isoforms to control striatal Sirt3-M1 groups. The differences were considered significant when the $\mathrm{p}$ values were less than 0.05 . 


\section{IV - RESULTS}

\section{IV/1 - COLD EXPOSURE}

There were no detectable changes in the levels of Pgc-1 $\alpha$ transcripts in the different brain areas after $200 \mathrm{~min}$ or $900 \mathrm{~min}$ cold $\left(4^{\circ} \mathrm{C}\right)$ exposure (Figure 2 and 3). After $200 \mathrm{~min}$ of cold exposure there were also no detectable changes in the levels of Sirt1 and Sirt3-M2 transcripts in any brain regions (Figure 4 A, B, C), but Sirt3-M1 levels elevated in the cortex (ctrl: 1.26 \pm 0.49; EX: $1.97 \pm 0.60 ; \mathrm{p}=0.036$; Figure 4 B), whereas Sirt3-M3 levels decreased in the cerebellum (ctrl: $0.16 \pm 0.05 ;$ EX: $0.10 \pm 0.03 ; p=0.027$; Figure 4 C). 900 min of cooling resulted in the relative decrease of cortical Sirt1 (ctrl: $1.14 \pm 0.31$; EX: $0.66 \pm 0.24 ; p=0.008$; Figure 4 E) and striatal Sirt3-M1 (ctrl: $1.04 \pm 0.30$; EX: $0.72 \pm 0.21 ; p=0.029$; Figure 4 D) expression levels.

\section{IV/2 - EXERCISE TRAINING}

After 5-day-long rotarod training there was not present any change in the Pgc-1 $\alpha$ isoforms in any of the investigated areas (Figure 5). However, the 12 day exercise training resulted in significant increases in all investigated isoforms (FL-Pgc-1 $\alpha$, NT-Pgc-1 $\alpha$, CNS-Pgc$1 \alpha$ and REF-Pgc-1 $\alpha$ ) mRNA expression in the cerebellum (FL-Pgc-1 $\alpha$ : ctrl: $1.32 \pm 0.20$; EX: $1.59 \pm 0.19 ; p=0.024 ;$ NT-Pgc-1 $\alpha$ : ctrl:0.29 $\pm 0.04 ;$ EX: $0.38 \pm 0.04 ; p=0.0002 ;$ CNS-Pgc$1 \alpha: \operatorname{ctrl}: 1.35 \pm 0.23$; EX: $1.80 \pm 0.32 ; p=0.003$, REF-PgcC-1 $\alpha$ : ctrl: $0.21 \pm 0.03$; EX: $0.30 \pm$ $0.02 ; \mathrm{p}=0.0003$; Figure $7 \mathrm{C}$ ). With regards to the striatum and the cortex, no other significant differences were detected (Figure 7 A, B). (The CNS-specific promoter was hardly detectable in the quadriceps muscle. However, the expression level of FL-Pgc-1 $\alpha$ (ctrl: $1.01 \pm 0.19$; EX: $3.19 \pm 1.25 ; p=0.003)$, NT-Pgc- $1 \alpha(\operatorname{ctrl}: 0.10 \pm 0.02 ; \mathrm{EX}: 0.50 \pm 0.19 ; \mathrm{p}=0.001)$ and REFPgc-1 $\alpha$ (ctrl: $1.00 \pm 0.11$; EX: $1.69 \pm 0.52 ; \mathrm{p}=0.016)$ mRNA was significantly elevated in the

quadriceps muscle after 5 days of training.) From the perspective of Sirtuins, after 5 days of rotarod training, cortical Sirt1 levels were found to be elevated (ctrl: $0.78 \pm 0.10$; EX: $0.97 \pm$ $0.16 ; \mathrm{p}=0.042$ ), but the other isoforms stayed unchanged (Figure 6). However, 12 days of exercise training resulted in the increase of both Sirt3-M1 and -M2 mRNA expression in the cerebellum (Sirt3-M1: ctrl: $0.79 \pm 0.18$; EX: $1.28 \pm 0.30 ; p=0.002 ;$ SIRT3-M2: ctrl: $0.33 \pm$ 
0.09; EX: $0.50 \pm 0.10 ; p=0.007$; Figure 7 F). We did not find differences in Sirt1 and Sirt3M3 levels in any other brain areas (Figure 7 D, E).

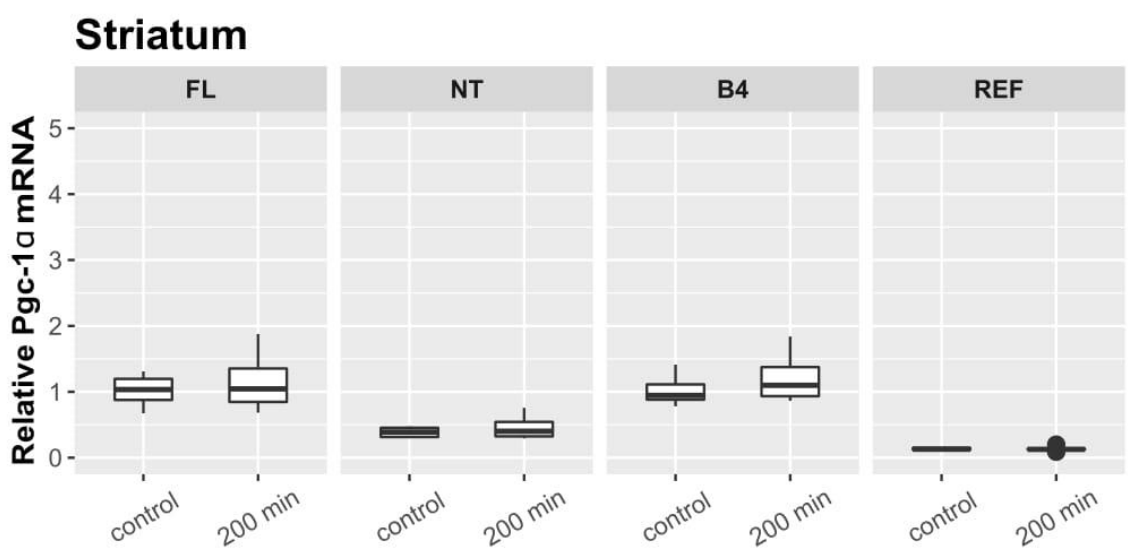

\section{Cortex}
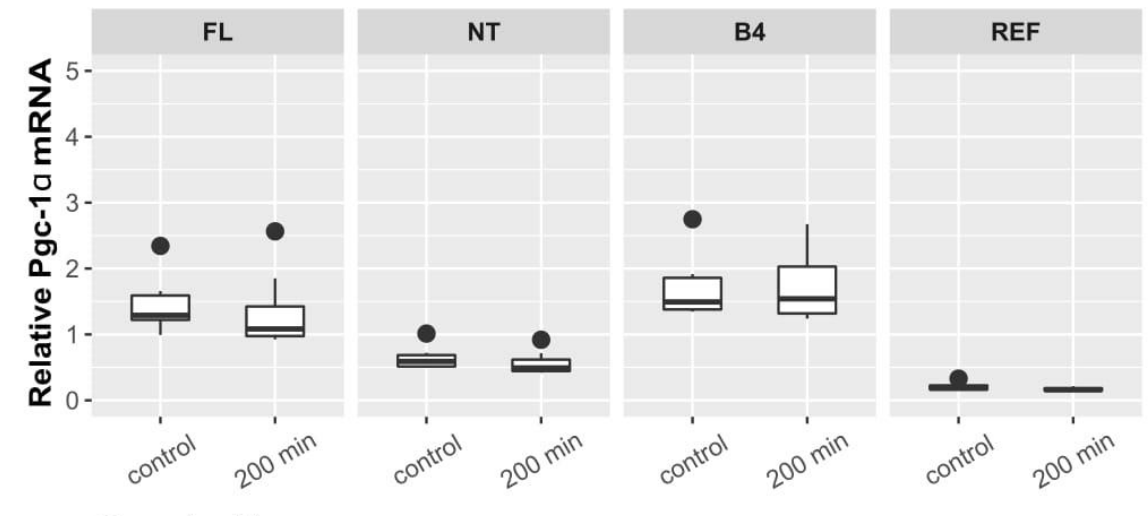

\section{Cerebellum}
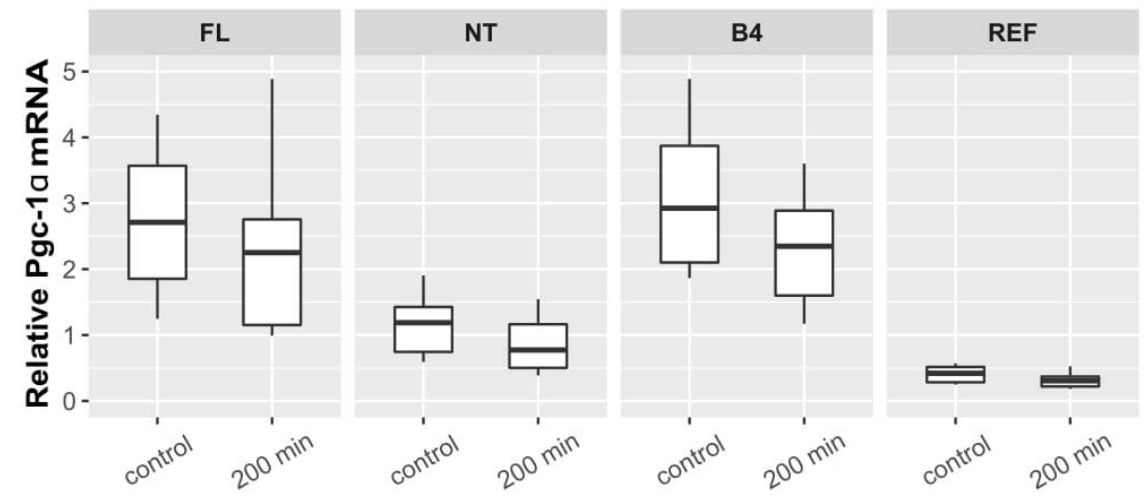

Figure 2. - Striatal, cortical and cerebellar relative mRNA expression levels of Pgc1-1 $\alpha$ isoforms in mice after 200 minutes of cold exposure $\left(4^{\circ} \mathrm{C}\right)$. Expression levels of the examined isoforms did not change. Values are plotted as medians and interquartile range; $200 \mathrm{~min}-200$ minutes cold exposure; $F L-$ FL-Pgc-1 $\alpha$; NT - NT-Pgc- $1 \alpha ; B 4$ - CNS-Pgc-1 $\alpha$; REF - REF-Pgc$1 \alpha$ (Salamon et al., 2019) 


\section{Striatum}

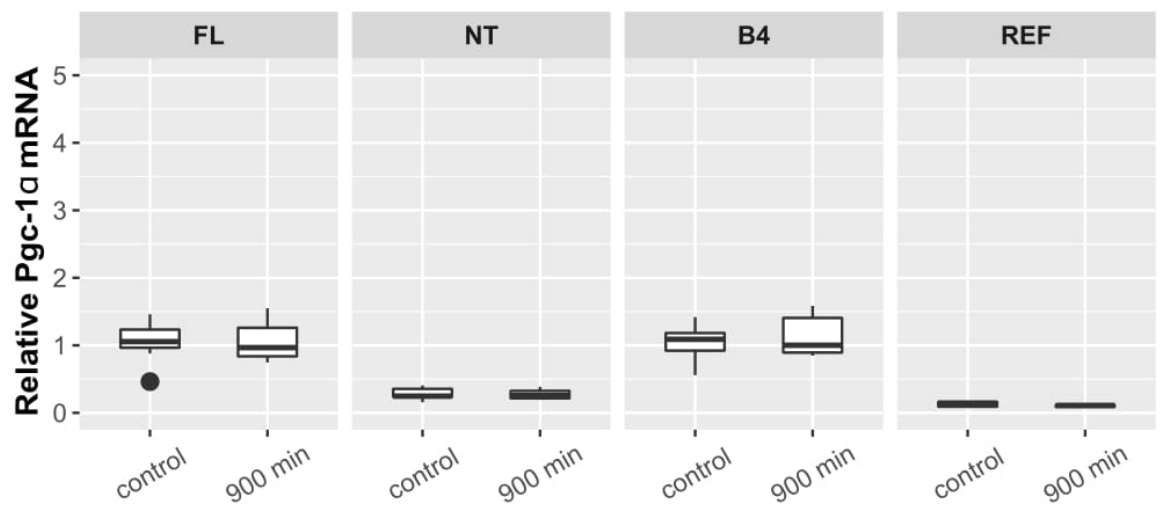

\section{Cortex}
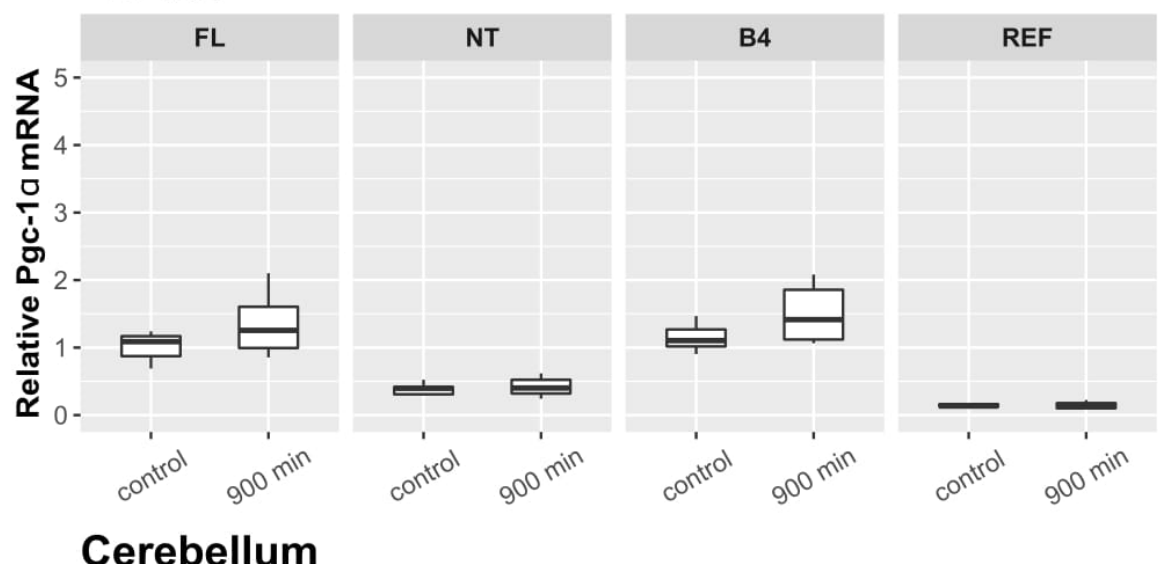

Cerebellum

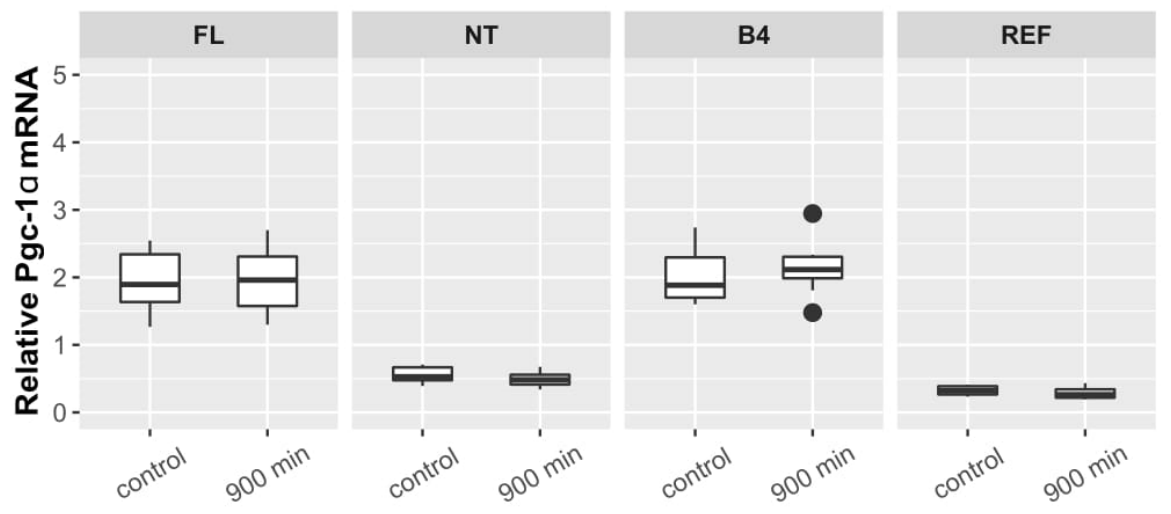

Figure 3. - Striatal, cortical and cerebellar relative mRNA expression levels of Pgc1-1 $\alpha$ isoforms in mice after 900 minutes of cold exposure $\left(4^{\circ} \mathrm{C}\right)$. Expression levels of the examined isoforms did not change. Values are plotted as medians and interquartile range; $900 \mathrm{~min}$ - 900 minutes cold exposure; FL - FL-Pgc-1 $\alpha ; N T$ - NT-Pgc-1 $\alpha$; B4 - CNS-Pgc-1 $\alpha$; REF - REF-Pgc-1 $\alpha$ (Salamon et al., 2019) 


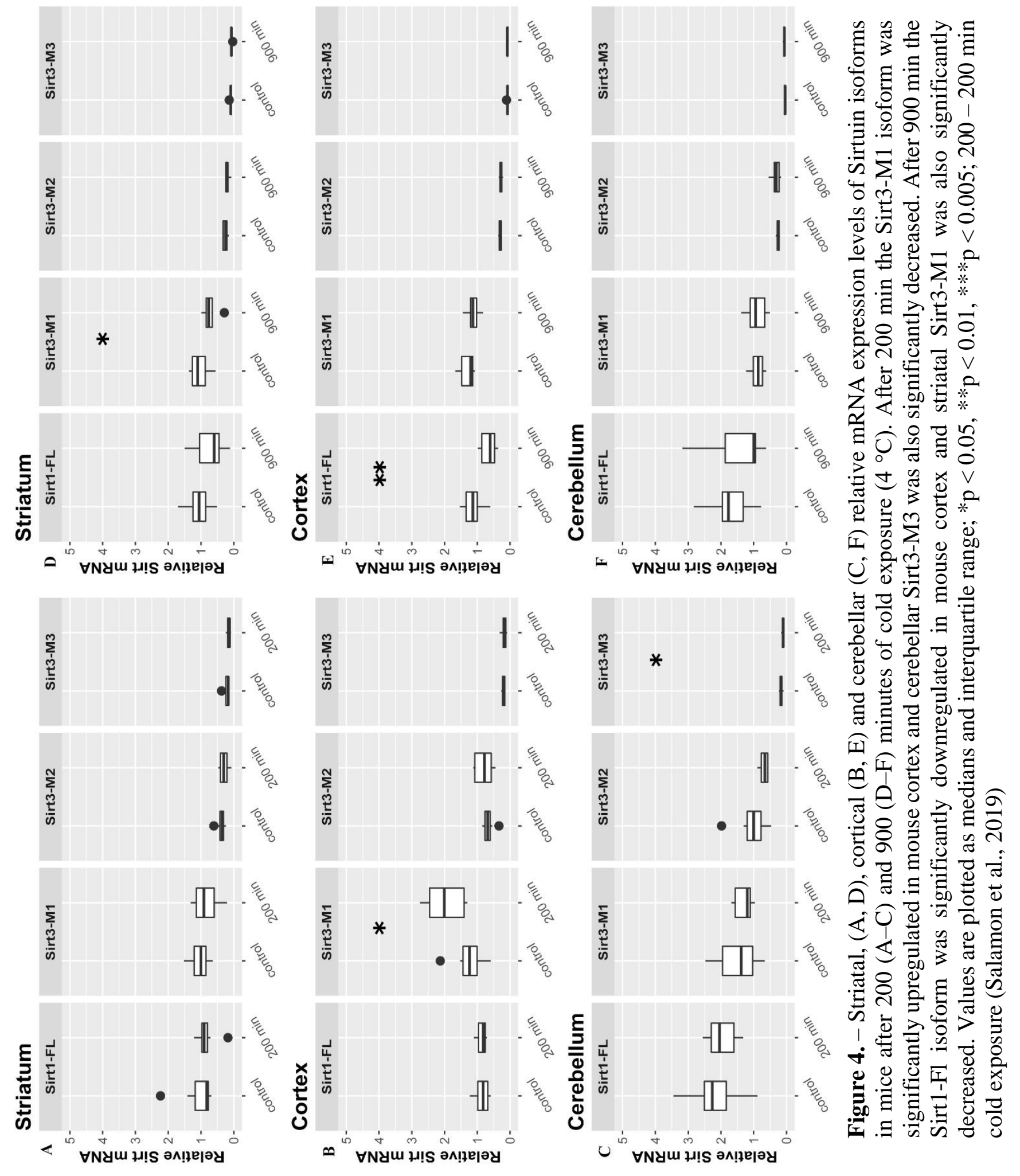




\section{Striatum}

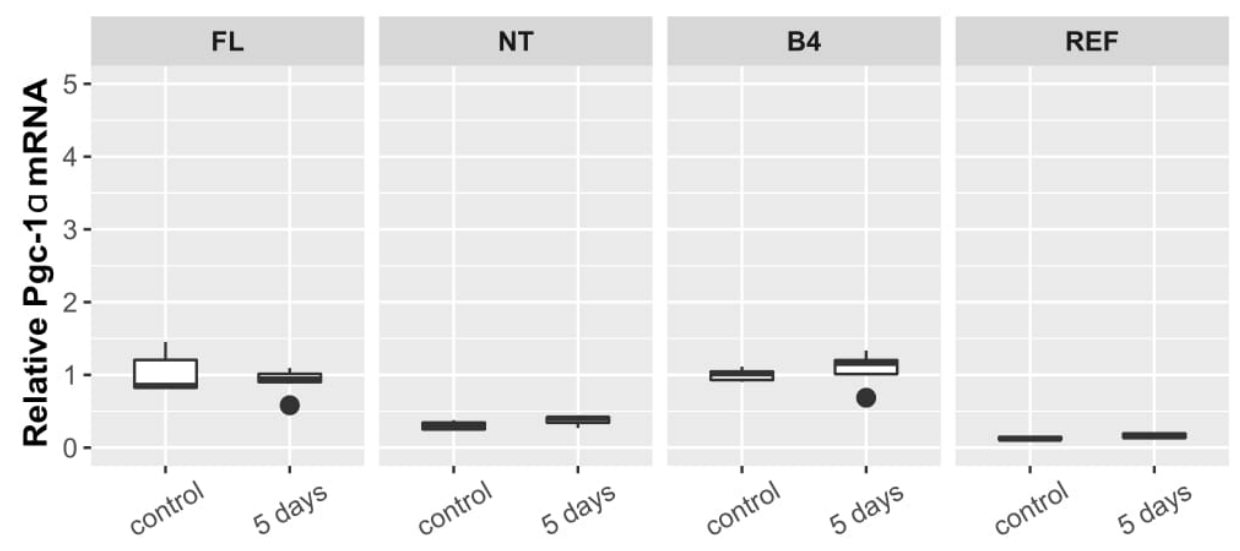

\section{Cortex}
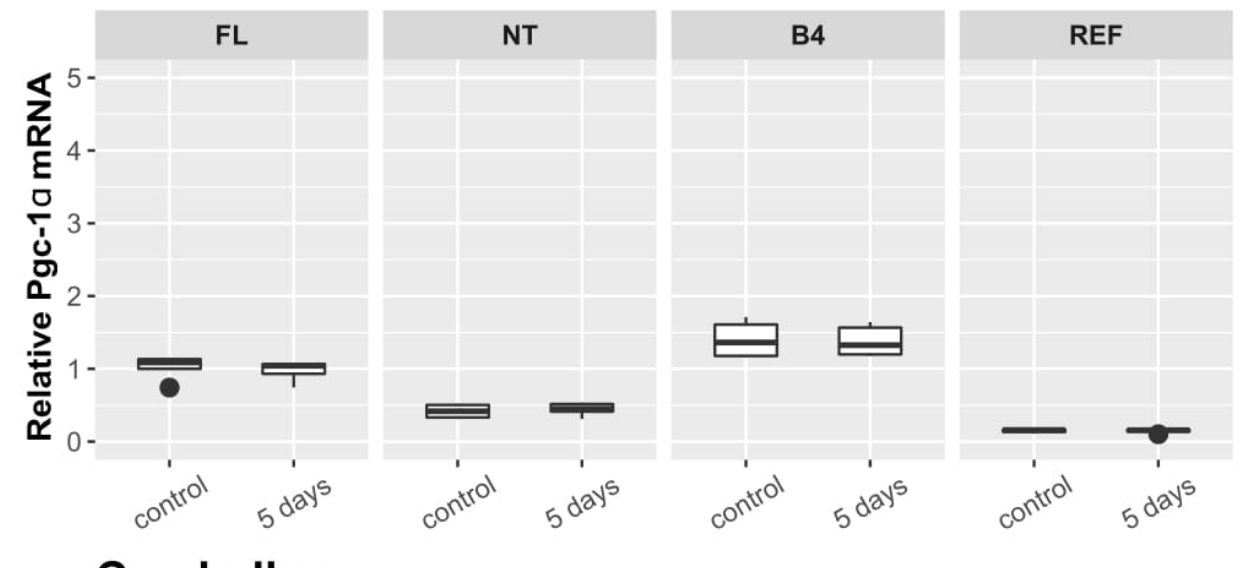

Cerebellum
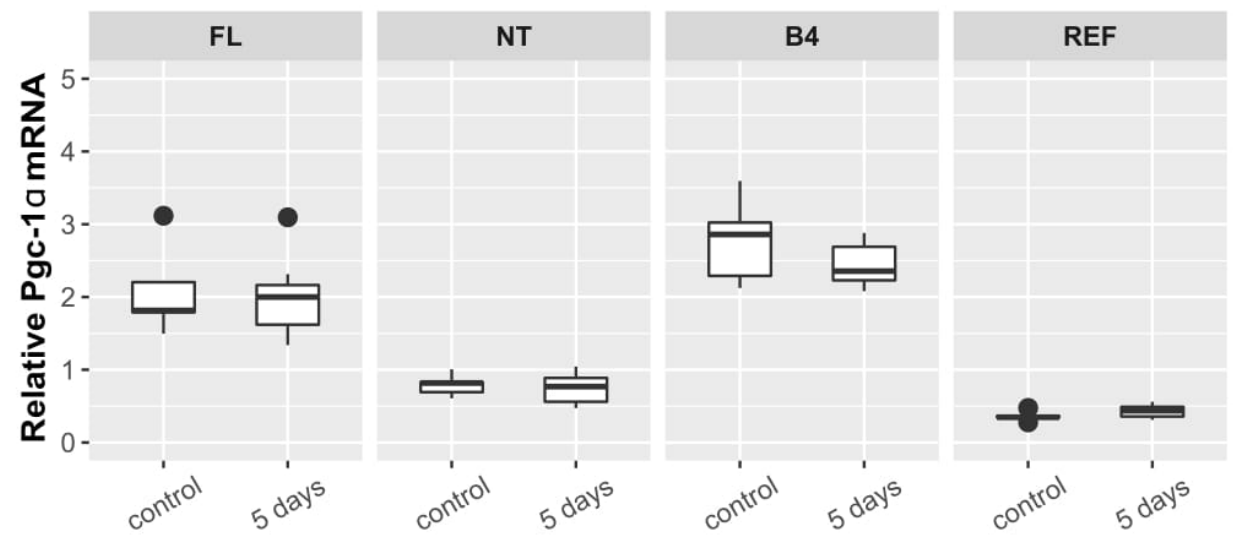

Figure 5. - Striatal, cortical and cerebellar relative mRNA expression levels of Pgc1-1 $\alpha$ isoforms in mice after 5 days of rotarod training (5 RPM). Expression levels of the examined isoforms did not change. Values are plotted as medians and interquartile range; 5 days -5 days rotarod training; $F L-$ FL-Pgc- $1 \alpha ; N T-$ NT-Pgc- $1 \alpha$; $B 4$ - CNS-Pgc-1 $\alpha$; REF - REF-Pgc-1 $\alpha$ (Salamon et al., 2019) 


\section{Striatum}

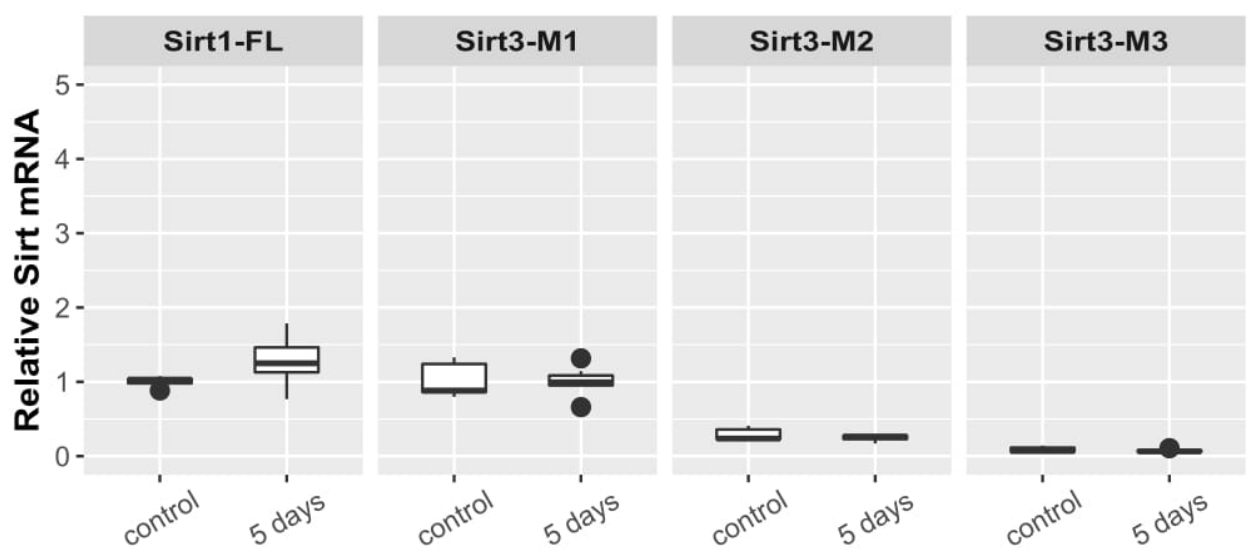

Cortex

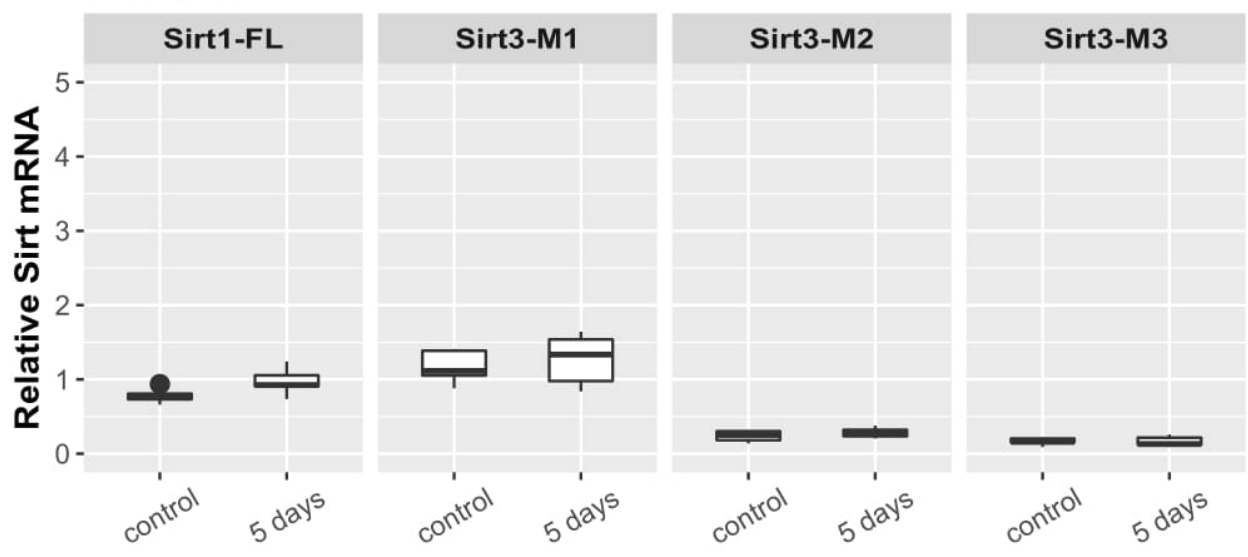

Cerebellum

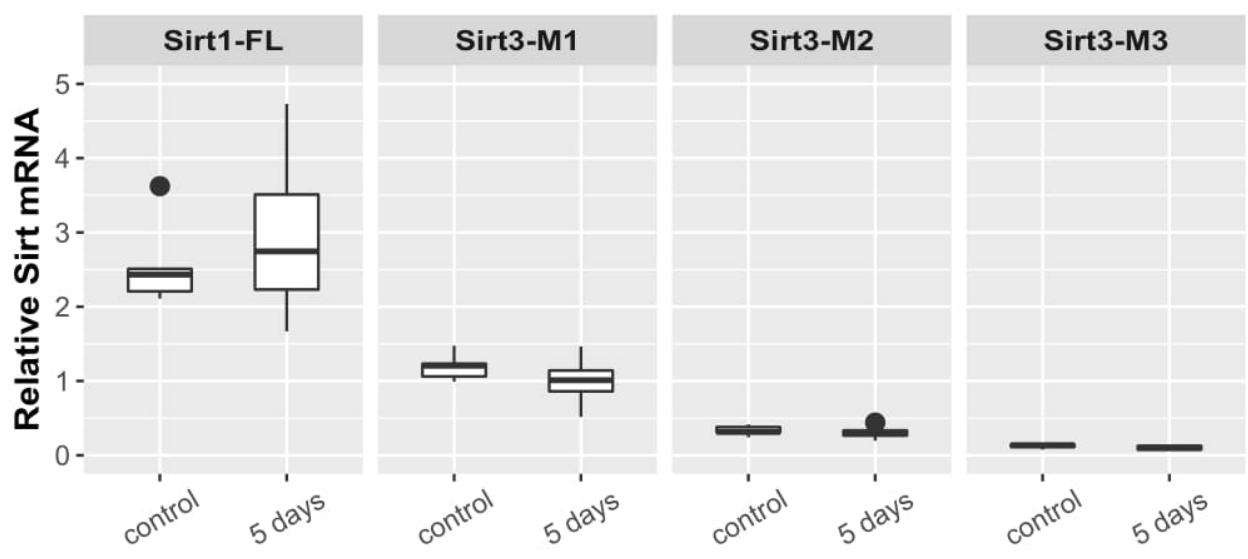

Figure 6. - Striatal, cortical and cerebellar relative mRNA expression levels of Sirtuin isoforms in mice after 5 days of rotarod training (5 RPM). Expression levels of the examined isoforms did not change. Values are plotted as medians and interquartile range; 5 days - 5 days rotarod training; $F L-$ FL-Pgc- $1 \alpha ; N T-$ NT-Pgc-1 $\alpha ; B 4-$ CNS-Pgc-1 $\alpha$; REF - REF-Pgc-1 $\alpha$ (Salamon et al., 2019) 


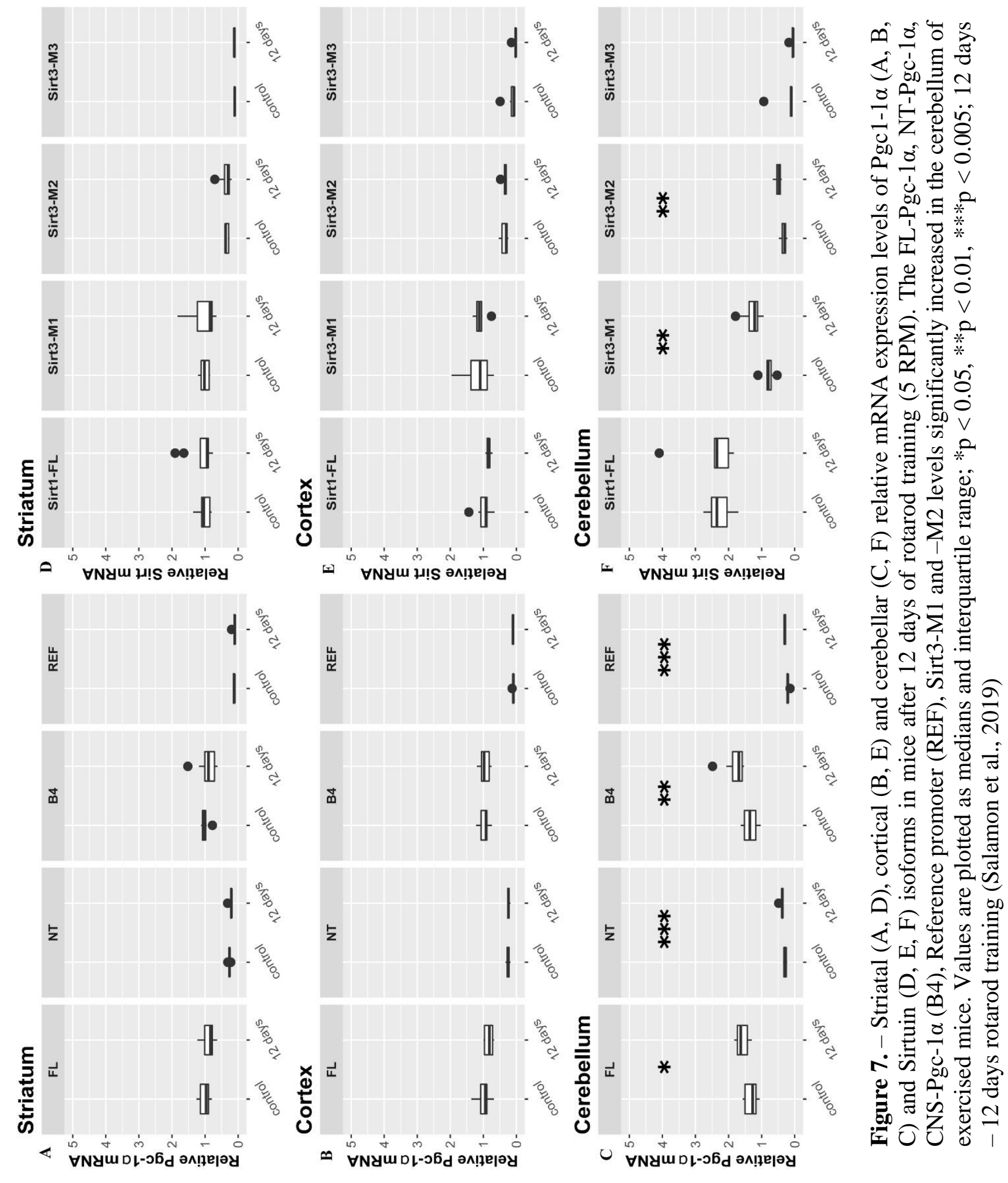




\section{IV/3 - MPTP TREATMENT}

Ninety minutes following the last MPTP injection of the acute treatment of MPTP, the FL-Pgc- $1 \alpha$ and NT-Pgc- $1 \alpha$ expression significantly increased in the striatum (FL-Pgc-1 $\alpha$ : ctrl: 0.97 (0.92-1.04); EX: 1.47 (1.21-1.83); p = 0.0048; NT-Pgc-1 $\alpha$ : ctrl: 0.44 (0.40-0.49); EX: 0.70 (0.56-0.78); p = 0.019), cortex (FL-Pgc-1 $\alpha$ : ctrl: 0.96 (0.91-1.06); EX: 1.23 (1.15-1.43), $\mathrm{p}=0.009$; NT-Pgc-1 $\alpha$ : ctrl: $0.46(0.43-0.48) ; \mathrm{EX}: 0.69(0.59-0.71) ; \mathrm{p}=0.0012)$ and cerebellum (FL-Pgc-1 $\alpha$ : ctrl: 1.50 (1.27-1.90); EX: 2.40 (2.07-2.76); p = 0.013; NT-Pgc1 $\alpha$ : ctrl: 0.67 (0.48-0.86); EX: 1.21 (1.14-1.44); p = 0.009) (Figure 8 A, B). Furthermore, MPTP-induced increases in CNS-Pgc-1 $\alpha$ expression were also significantly larger in all investigated brain regions compared to the controls (striatum: ctrl: 1.03 (0.88-1.11); EX: 1.38 (1.34-1.78); p = 0.0069; cortex: ctrl: 0.91 (0.80-0.98); EX: 1.41 (1.24-1.42); $\mathrm{p}=0.0048$; cerebellum: ctrl: 1.51 (1.20-1.98); EX: 2.77 (2.34-3.17); p = 0.019) (Figure 8 C). However, there was not any difference between the REF-Pgc-1 $\alpha$ levels in the striatum (ctrl: $0.11(0.10-0.12)$; EX: 0.11 (0.95-0.12)); cortex (ctrl: $0.11(0.11-0.12)$; EX: 0.09 (0.08-0.10)) and cerebellum (ctrl: 0.21 (0.20-0.29); EX: 0.28 (0.24-0.29)) of MPTP-treated and control mice (Figure 8 D). One week following the last injection in the acute treatment regimen, there was not any significant change either in the FL-, NT-, CNS-, or in the REF-Pgc-1 $\alpha$ levels between the control and the MPTPtreated animals in any brain area (Figure 9).

There were no detectable changes in the levels of Sirtuin transcripts in the different brain areas after ninety minutes following the last MPTP injection (Figure 10). However, 1 week following the last injection a slight significance was found in all of the cerebellar Sirt3 isoforms (Sirt3M1: ctrl: $1.11 \pm 0.29$; EX: $1.44 \pm 0.14 ; \mathrm{p}=0.015$; Sirt3-M2: ctrl: $0.33 \pm 0.08$; EX: $0.44 \pm 0.05$; $\mathrm{p}=0.021 ;$ Sirt3-M3: ctrl: $0.03 \pm 0.01 ; \mathrm{EX}: 0.044 \pm 0.01 ; \mathrm{p}=0.018$; Figure 11). However, these significances disappeared using the Bonferroni correction (therefore, this is not indicated in the figure). 
A

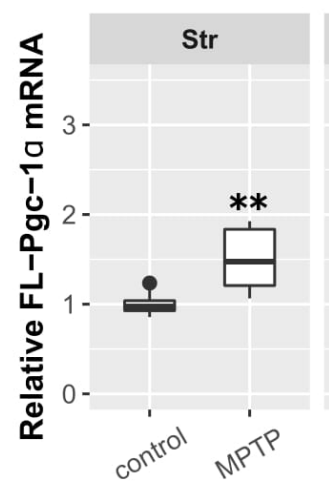

C

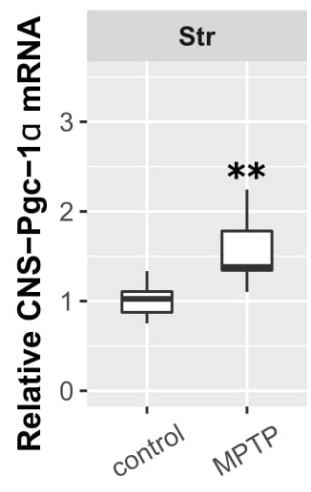

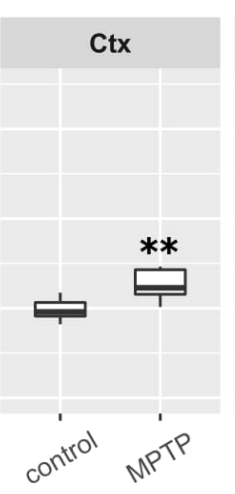
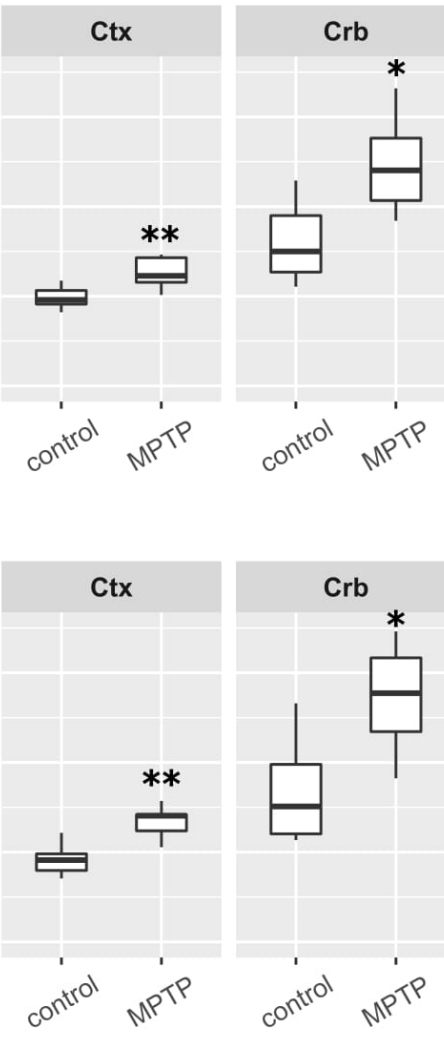

B
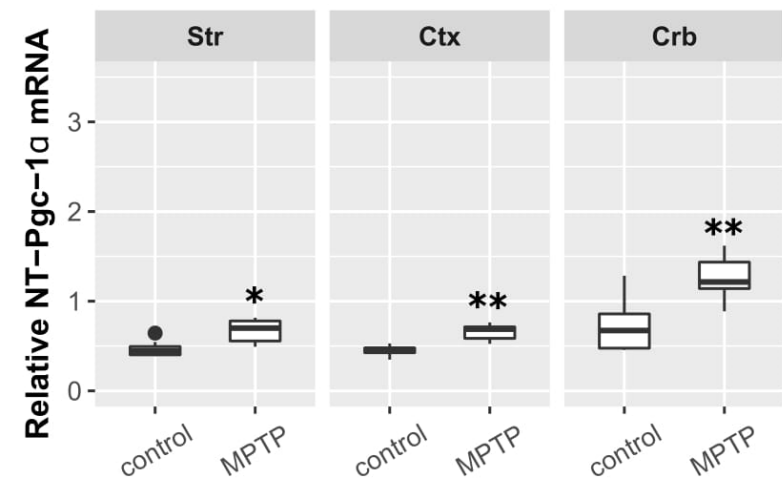

D

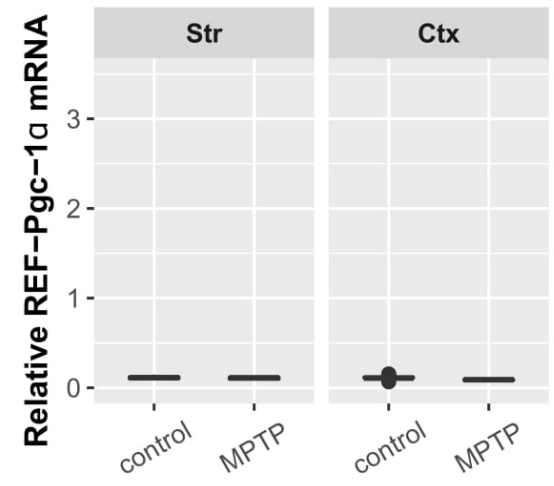

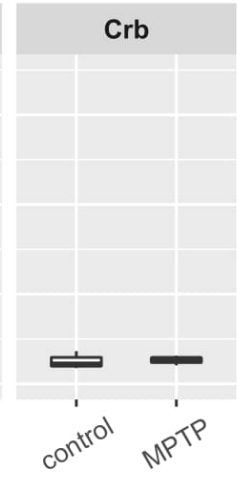

Figure 8. - The relative mRNA expression of Pgc- $1 \alpha$ isoforms in the striatum, cortex and the cerebellum of mice 90 min after acute MPTP intoxication. The FL-Pgc-1 $\alpha$, NT-Pgc- $1 \alpha$ and CNS-Pgc-1 $\alpha$ levels were significantly increased in the striatum, cortex and the cerebellum of MPTP-treated mice. (A, B, C respectively). The RefPgc-1 $\alpha$ expression did not change in any brain areas of MPTP-treated mice compared to the controls (D). Values are plotted as medians and interquartile range; *p $<0.05$, **p < 0.01; MPTP MPTP-treated; Str striatum, Ctx cortex, Crb cerebellum. (Török et al., 2017) 
A

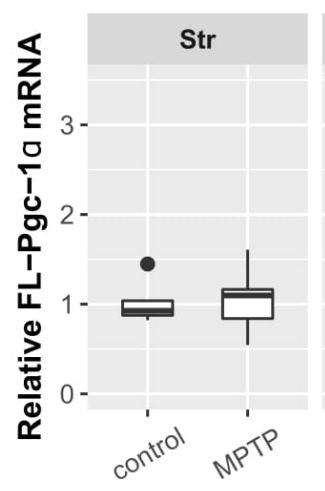

C
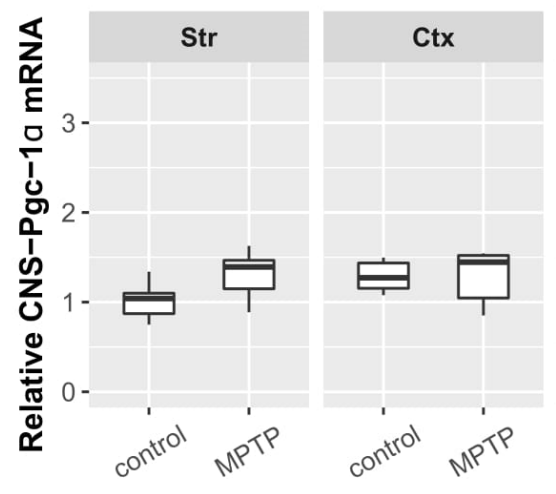

B
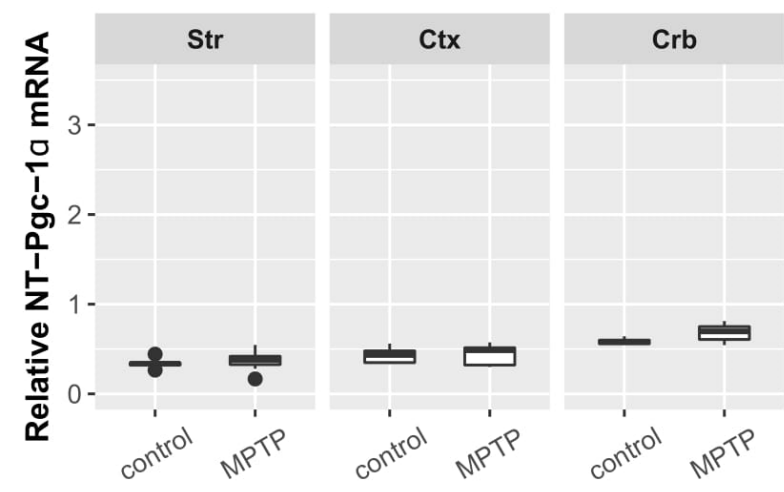

D

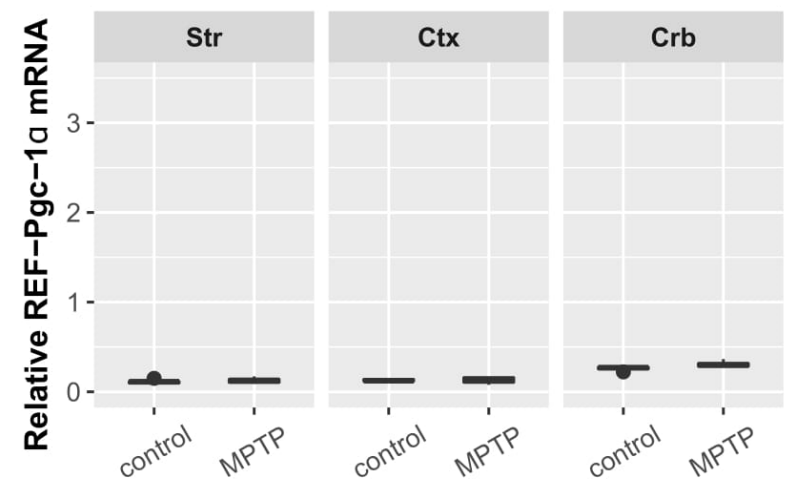

Figure 9. - The relative mRNA expression of Pgc-1 $\alpha$ isoforms in the striatum, cortex and the cerebellum of mice 7 days after acute MPTP intoxication. The expression levels of the Pgc-1 $\alpha$ isoforms did not change in any brain areas of MPTP-treated mice (A-D). Values are plotted as medians and interquartile range; MPTP MPTP-treated; Str striatum, Ctx cortex, Crb cerebellum. (Török et al., 2017)

\section{IV/4 - N171-82Q EXPERIMENT}

We could not detect any significant difference between male and female mice regarding the expression any of the assessed Sirtuin isoforms either in the wt or in the tg groups, so they were pooled for further analyses. Furthermore, in respect of Sirt1 and Sirt3 expression, no interaction was found between the presence of the transgene and aging. Focusing on their separate effects, there was a significant elevation of Sirt1 expression in all the cortical and cerebellar samples of 8-, 12- and 16-week-old tg animals compared to wt mice (cortex (8-weekold: $\mathrm{p}=0.0029 ; 12$-week-old: $\mathrm{p}=0.0018$; 16-week-old: $\mathrm{p}=0.0029$ ); cerebellum (8-week-old: 
$\mathrm{p}=0.0052 ; 12$-week-old: $\mathrm{p}=0.0054 ; 16$-week-old: $\mathrm{p}=0.0065$ ), but not in the striatum (Figure 12). Regarding the effect of aging on Sirt1 expression levels, we detected significant increase only in the cerebellum of tg group at 16 weeks of age (8-vs. 16 -week-old: $p=0.0245 ; 12$ - vs. 16-week-old: $\mathrm{p}=0.0316$ ). There was no detectable change in Sirt3-M1 expression in the striatum and cortex of any age groups (8-, 12-, 16-week-old). In contrast, there was a clear elevation of Sirt3-M1 mRNA expression in cerebellar samples of all age groups of tg animals compared to wt mice (8-week-old: $\mathrm{p}=0.0024 ; 12$-week-old: $=0.0024 ; 16$-week-old: $\mathrm{p}=$ 0.0024; Figure 13). We could not observe age-related effect in the Sirt3-M1 isoform in either group. Regarding Sirt3-M2 we could not detect any difference between wt and tg groups in the striatal and cortical samples, but we could identify a significant elevation in the cerebellum of tg animals in each age group compared to wt controls (8-week-old: $p=0.0021 ; 12$-week-old: $p$ $=0.0012$; 16-week-old: $\mathrm{p}=0.0021$; Figure 14). When assessing the effect of aging on Sirt3M2 expression levels, we detected significant decrease only in the cortex of wt group at 16 weeks of age (8- vs. 16-week-old: $\mathrm{p}=0.038 ; 12$ - vs. 16-week-old: $\mathrm{p}=0.038$ ). The expression of Sirt3-M3 elevated in each striatal and cerebellar tg group, but only in the 16-week-old group in the tg cortex compared to wt mice (striatum (8-week-old: $\mathrm{p}=0.0097$; 12-week-old: $\mathrm{p}=$ 0.0054; 16-week-old: $\mathrm{p}=0.0097$ ); cortex (16-week-old: $\mathrm{p}=0.0032$ ); cerebellum (8-week-old: $\mathrm{p}=0.002$; 12-week-old: $\mathrm{p}=0.0006 ; 16$-week-old: $\mathrm{p}=0.0016$ ) (Figure 15). In the striatal Sirt3M3 samples there was detectable decrease of expression by 12 weeks of age in both wt and tg animals (8- vs. 12-week-old (wt-wt): $\mathrm{p}=0.0243 ; 8$ - vs. 12-week-old (tg-tg): $\mathrm{p}=0.036$ ). 


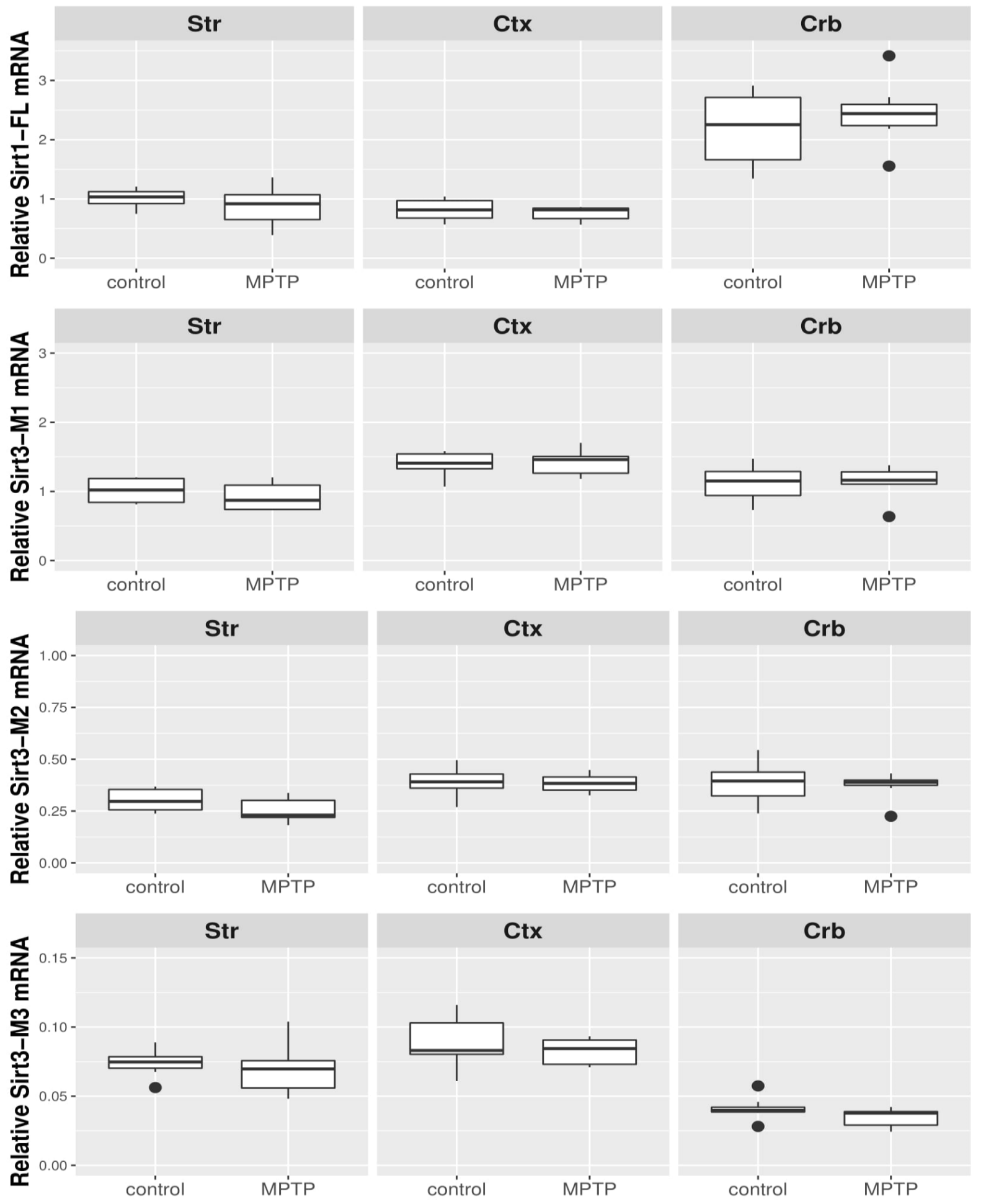

Figure 10. - The relative mRNA expression of Sirtuin isoforms in the striatum, cortex and the cerebellum of mice 90 min after acute MPTP intoxication. The expression levels of the Sirtuin isoforms did not change in any brain areas of MPTP-treated mice.Values are plotted as medians and interquartile range; MPTP MPTP-treated; Str striatum, $C t x$ cortex, $C r b$ cerebellum. (unpublished results) 


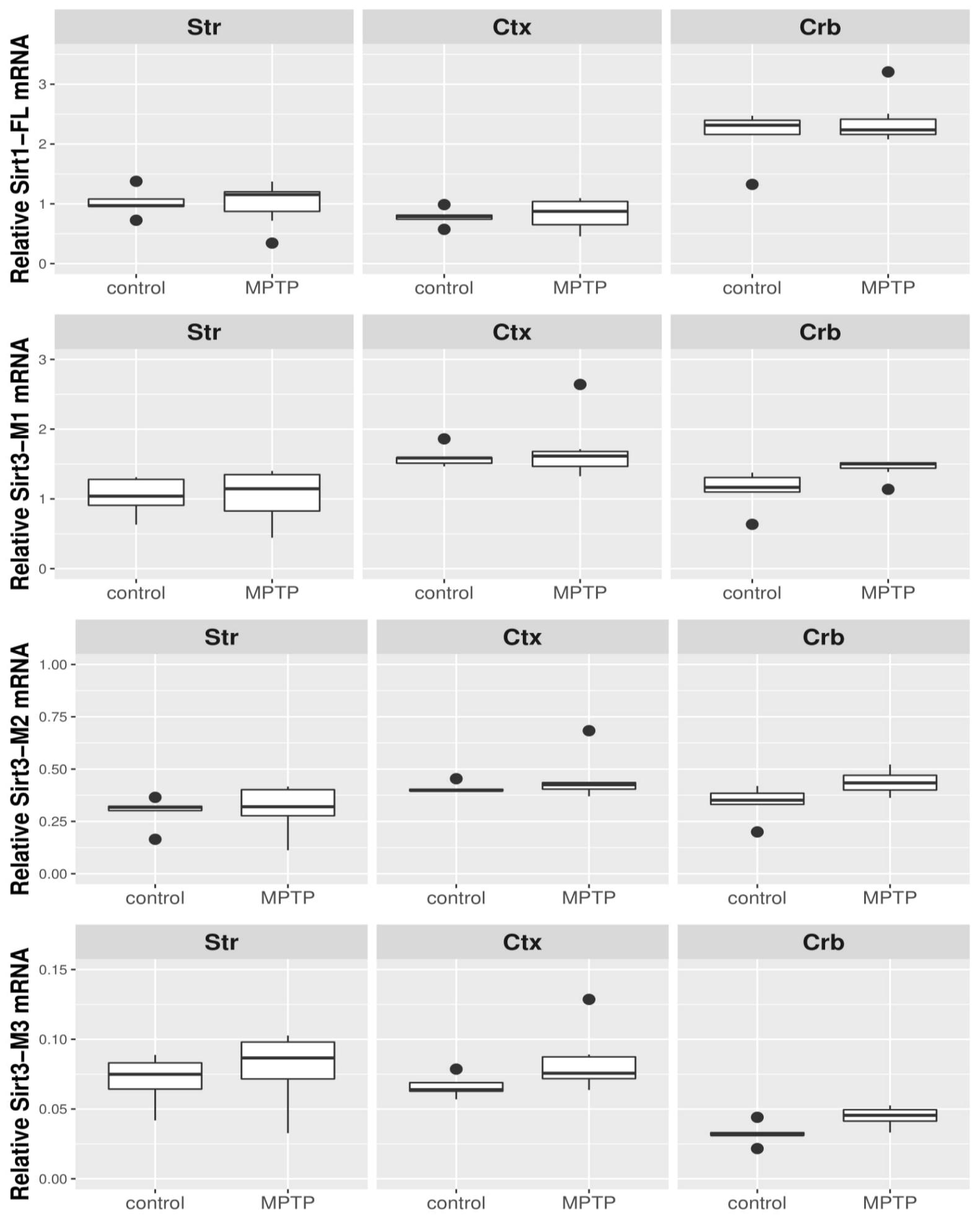

Figure 11. - The relative mRNA expression of Sirtuin isoforms in the striatum, cortex and the cerebellum of mice 7 days after acute MPTP intoxication. The expression levels of the Sirtuin isoforms did not change in any brain areas of MPTP-treated mice.Values are plotted as medians and interquartile range; MPTP MPTP-treated; Str striatum, Ctx cortex, $\mathrm{Crb}$ cerebellum. (unpublished results) 
a

Striatum
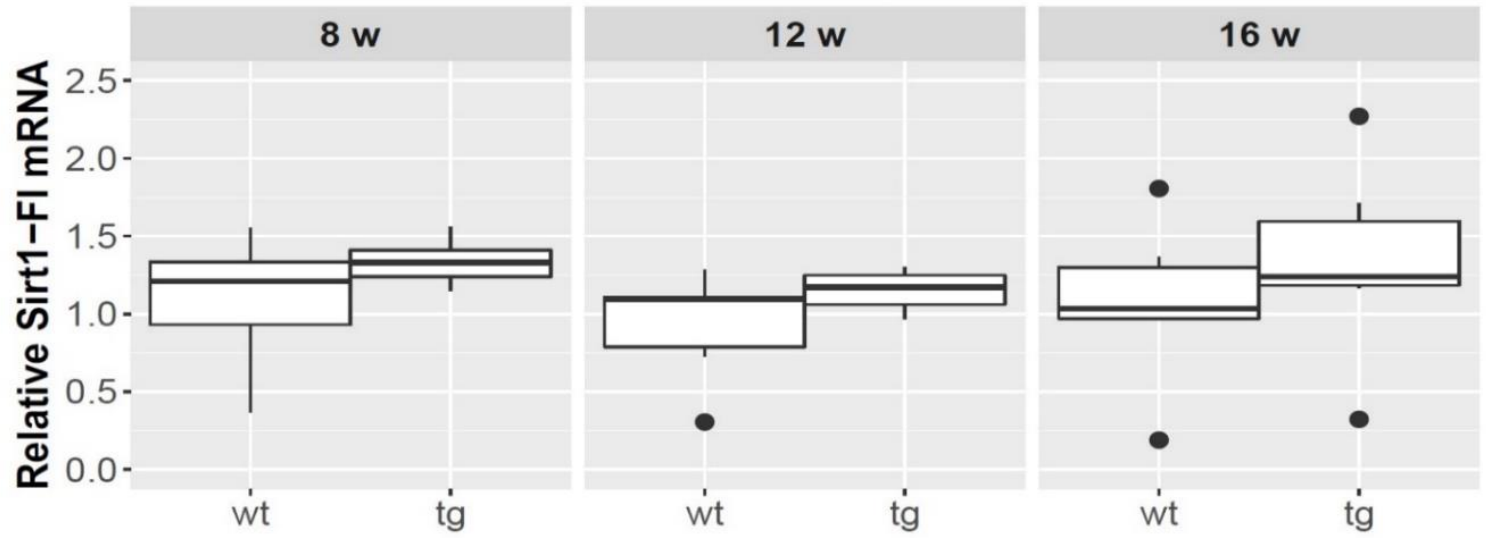

b
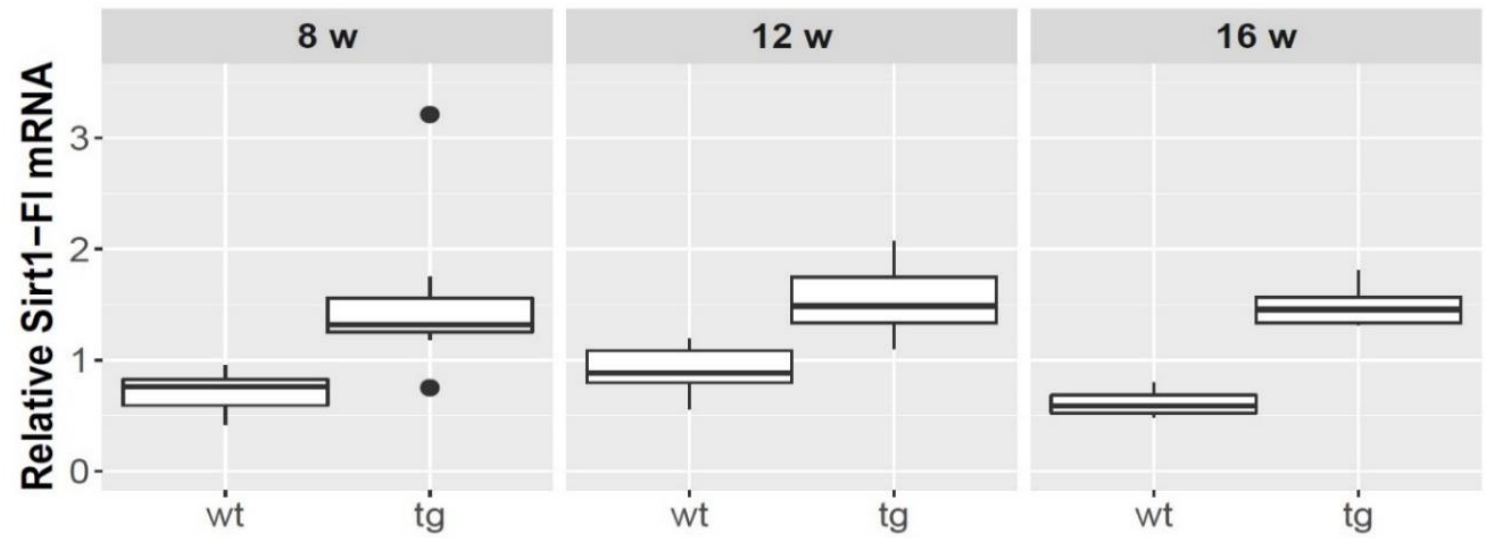

C

\section{Cerebellum}
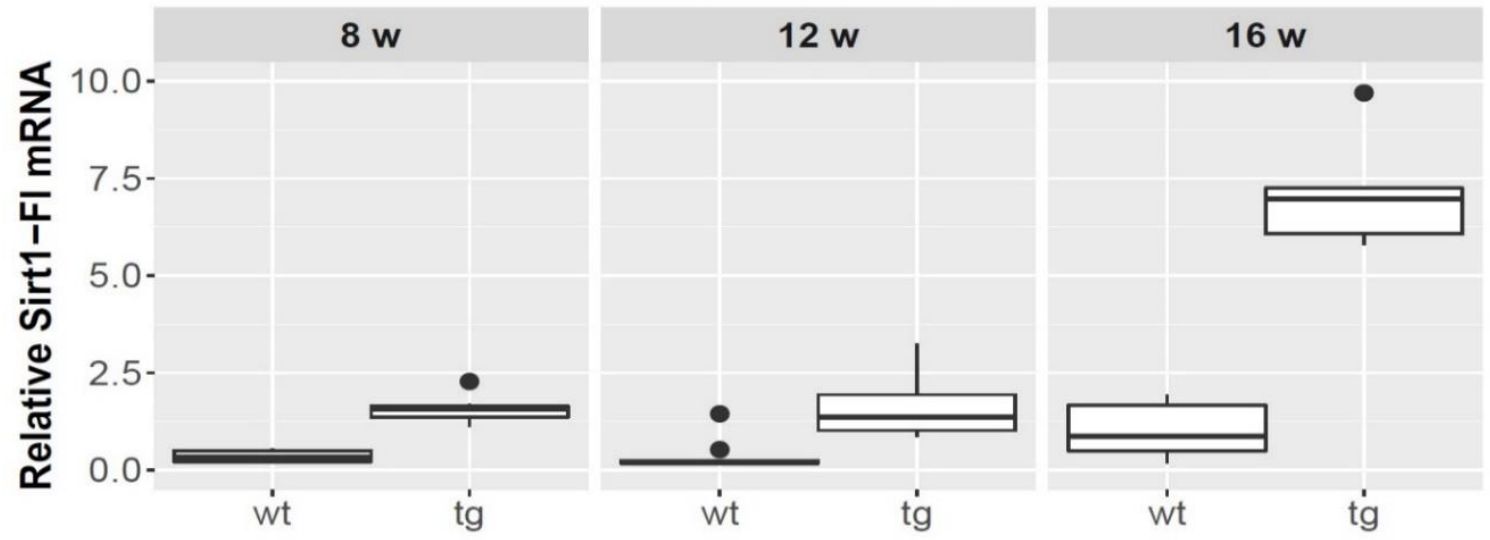

Figure 12. - Relative mRNA expression level of Sirt1-Fl in the striatum (a), cortex (b) and cerebellum (c) of N171-82Q transgenic and B6C3 wild-type mice of three age groups. The Sirt1-Fl level significantly elevated in all cortical and cerebellar samples in each age group of tg animals compared to wt mice. Aging caused a significant increase only in the cerebellum of tg group by 16 weeks of age. For clarity, the levels of significance were indicated separately in Figure 16. in a special table format. Values are plotted as medians and interquartile ranges; $\operatorname{tg}=$ transgenic, $w \mathrm{t}=$ wild-type, $\mathrm{w}=$ weeks 
a

\section{Striatum}
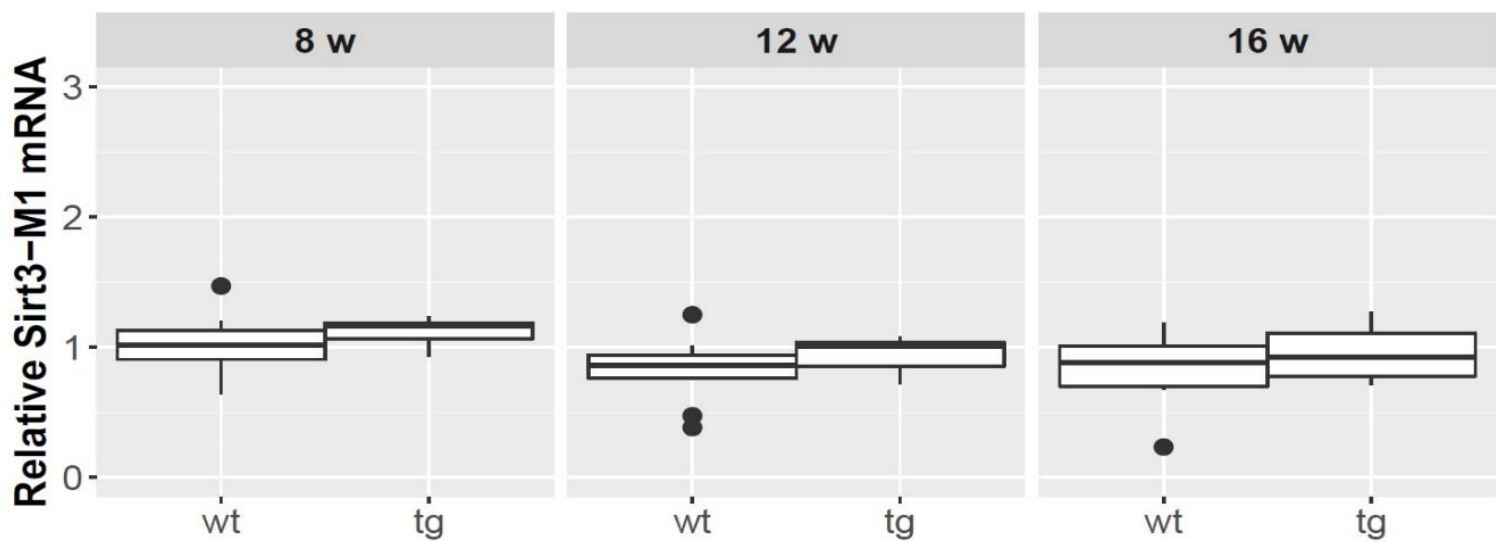

b

Cortex
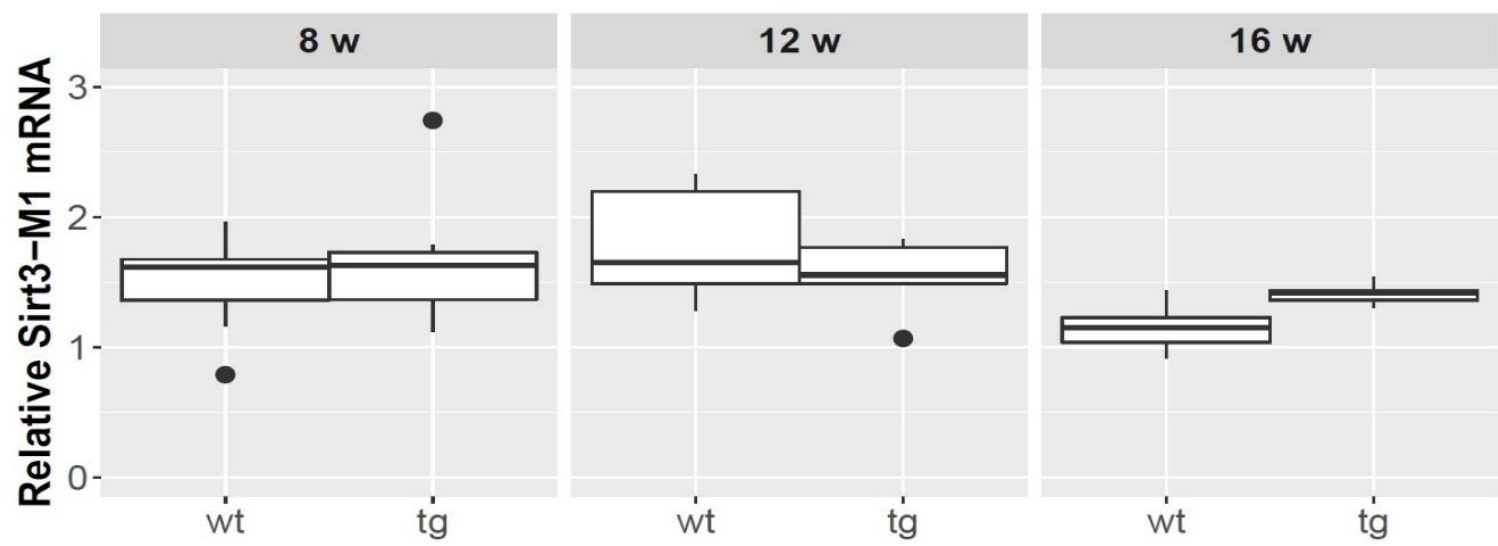

C

\section{Cerebellum}
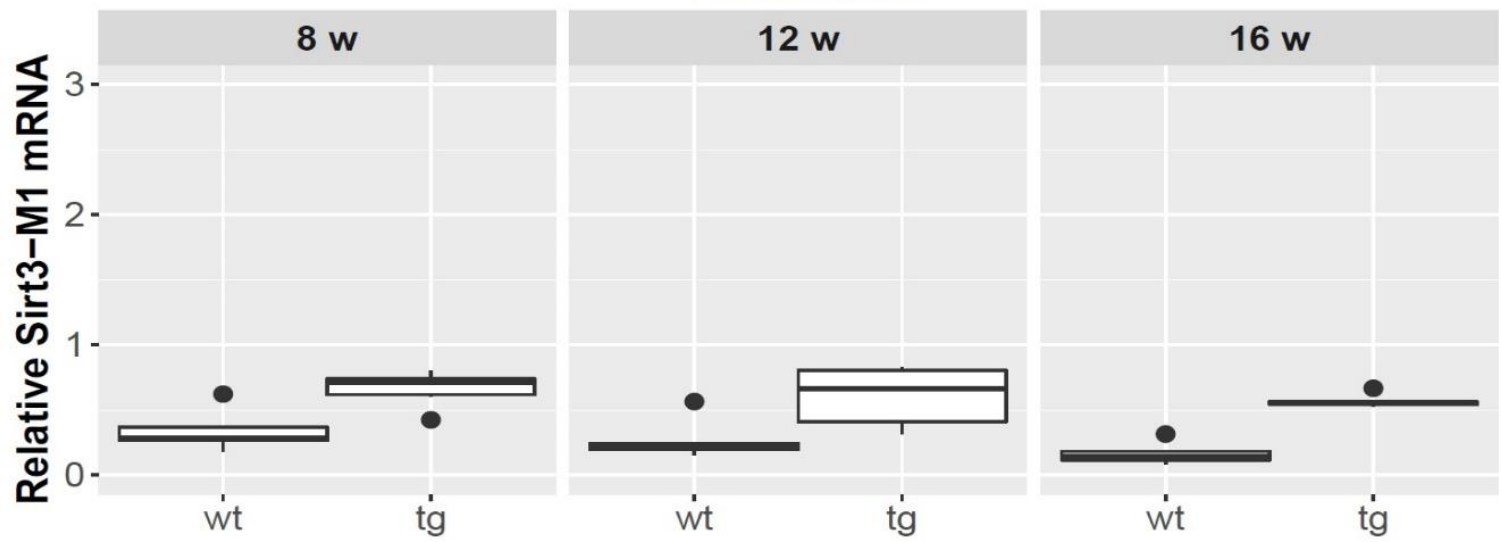

Figure 13. - Relative mRNA expression level of Sirt3-M1 in the striatum (a), cortex (b) and cerebellum (c) of N171-82Q transgenic and B6C3 wild-type mice of three age groups. The Sirt3-M1 level was significantly elevated in all cerebellar samples in each age group of tg animals compared to wt mice. For clarity, the levels of significance were indicated separately in Figure 16. in a special table format. Values are plotted as medians and interquartile ranges; $\operatorname{tg}=$ transgenic, $\mathrm{wt}$ = wild-type, $\mathrm{w}=$ weeks 
a
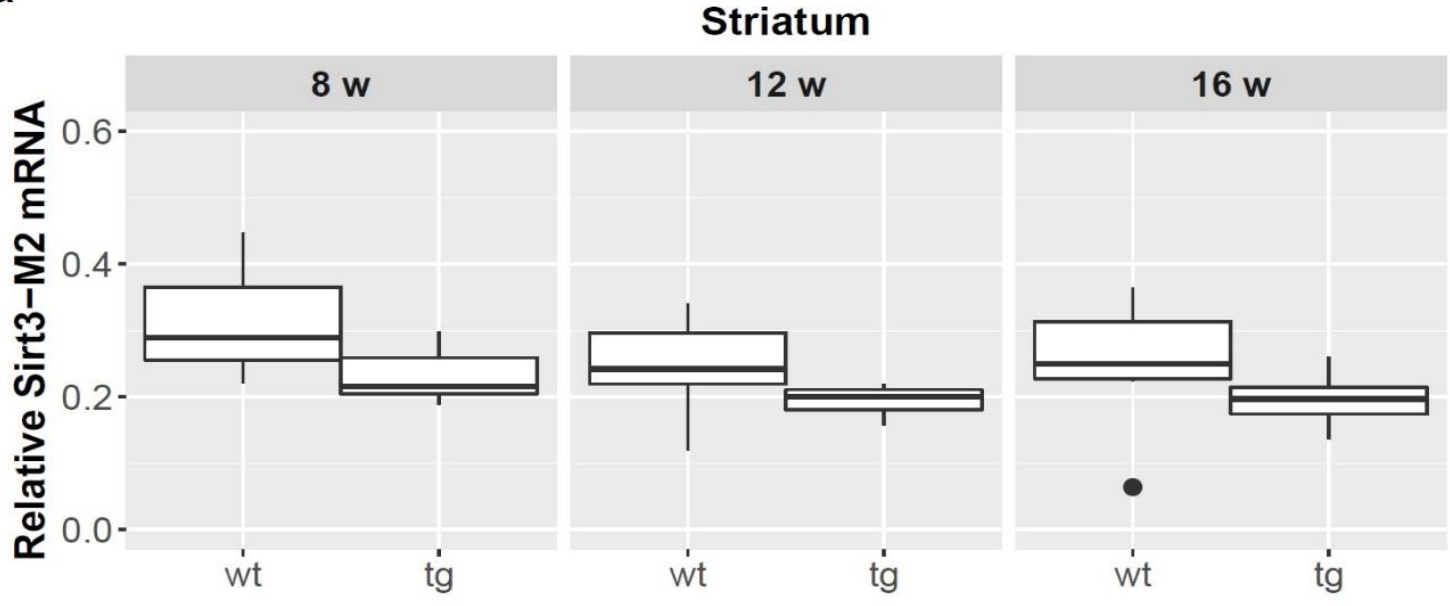

b
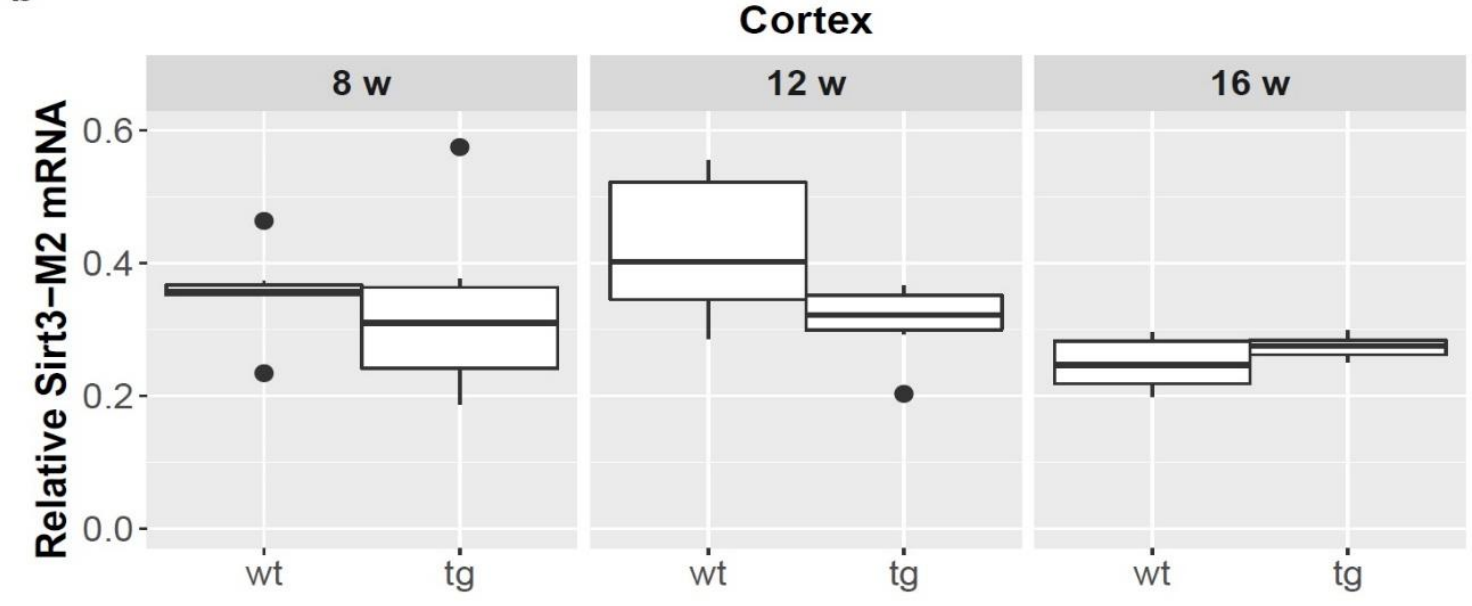

C
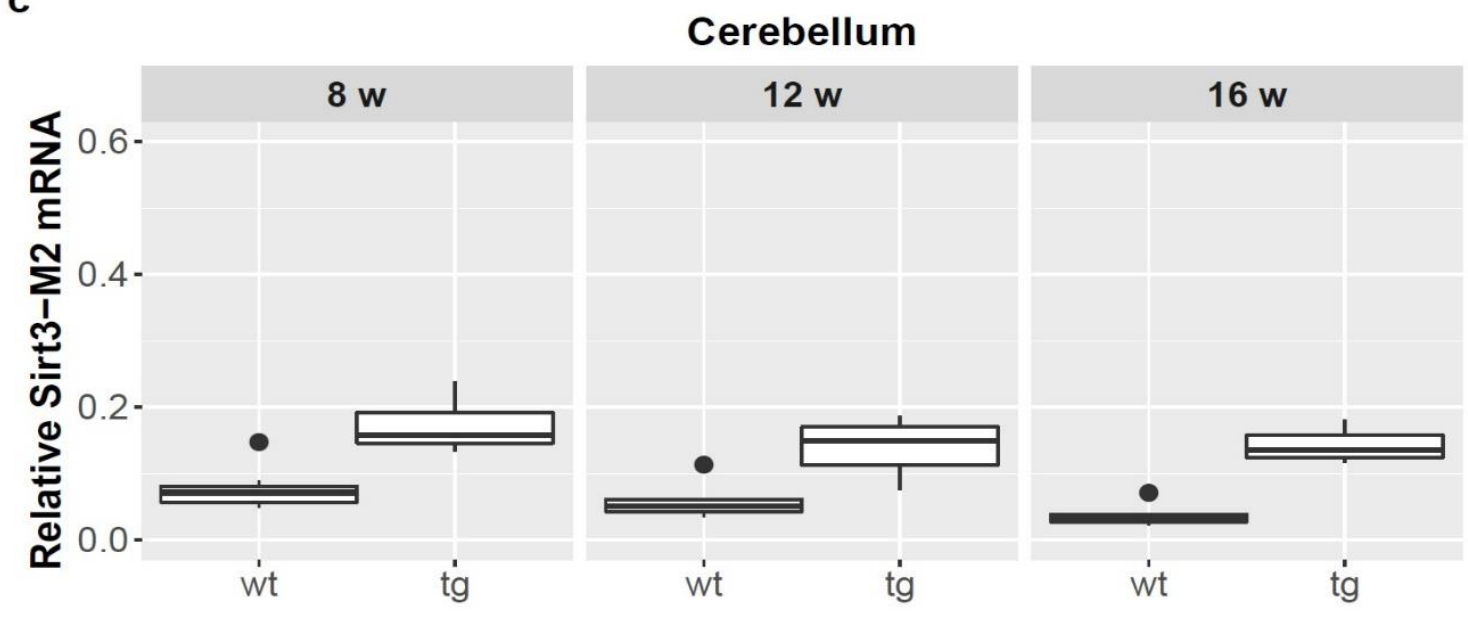

Figure 14. - Relative mRNA expression level of Sirt3-M2 in the striatum (a), cortex (b) and cerebellum (c) of N171-82Q transgenic and B6C3 wild-type mice of three age groups. The Sirt3-M2 level was significantly elevated in all cerebellar samples in each age group of tg animals compared to wt mice. Aging caused a significant decrease only in the cortex of wt group by 16 weeks of age. For clarity, the levels of significance were indicated separately in Figure 16. in a special table format. Values are plotted as medians and interquartile ranges; $\operatorname{tg}=$ transgenic, $w t=$ wild-type, $w=$ weeks 
a

\section{Striatum}
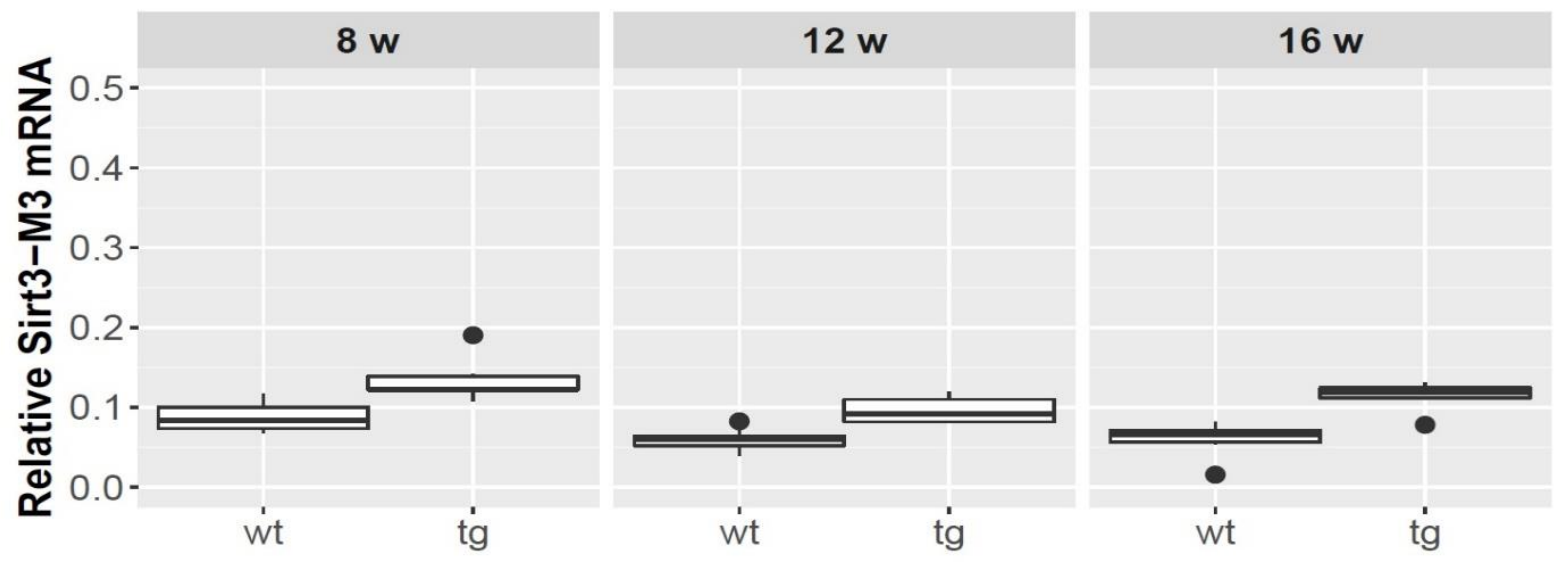

b
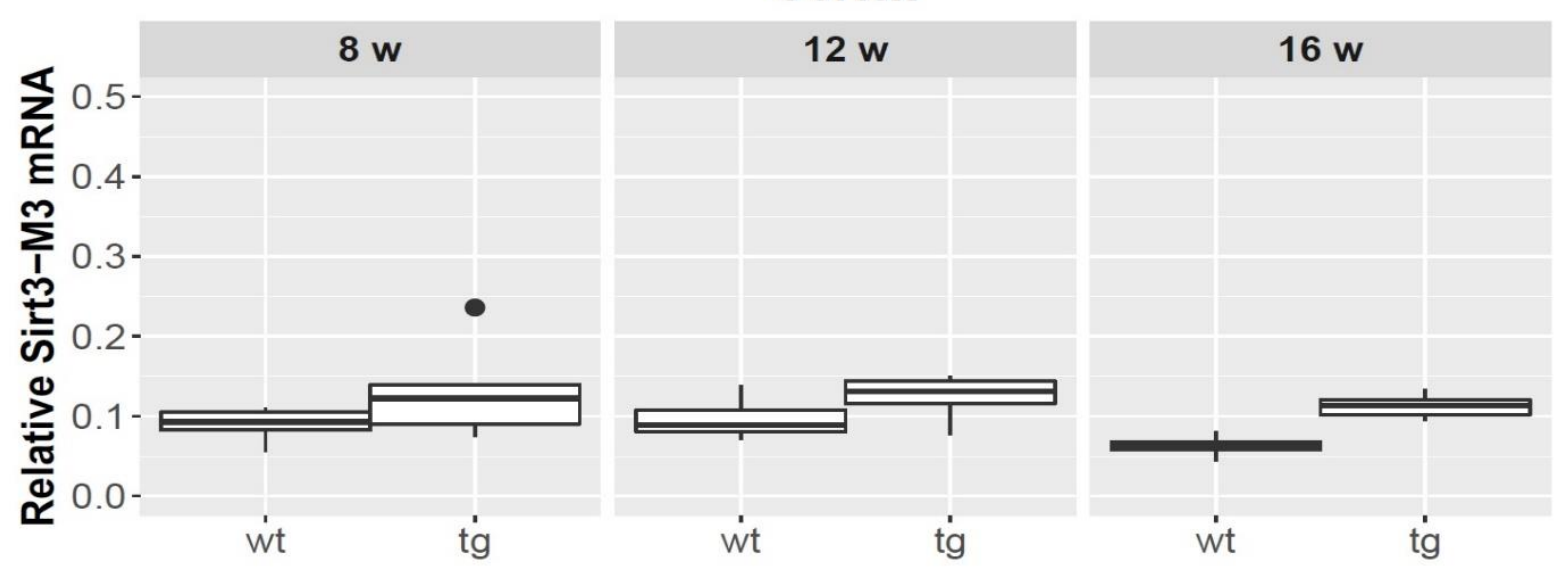

C
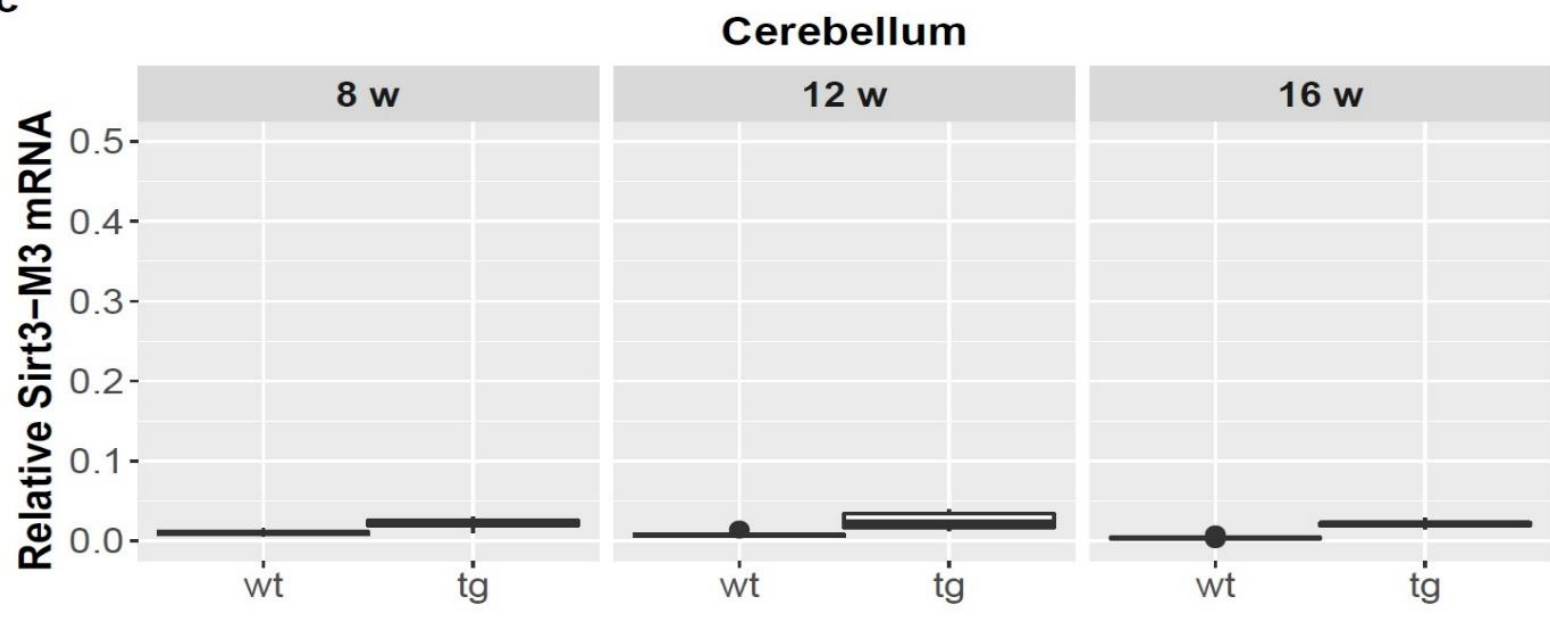

Figure 15. - Relative mRNA expression level of Sirt3-M3 in the striatum (a), cortex (b) and cerebellum (c) of N171-82Q transgenic and B6C3 wild-type mice of three age groups. The Sirt3-M3 level was significantly elevated in all striatal and cerebellar samples in each age group of tg animals compared to wt mice. The striatal expression decreased significantly by 12 weeks of age in both wt and tg animals. For clarity, the levels of significance were indicated separately in Figure 16. in a special table format. Values are plotted as medians and interquartile ranges; $\operatorname{tg}=$ transgenic, $\mathrm{wt}=$ wild-type, $\mathrm{w}=$ weeks 


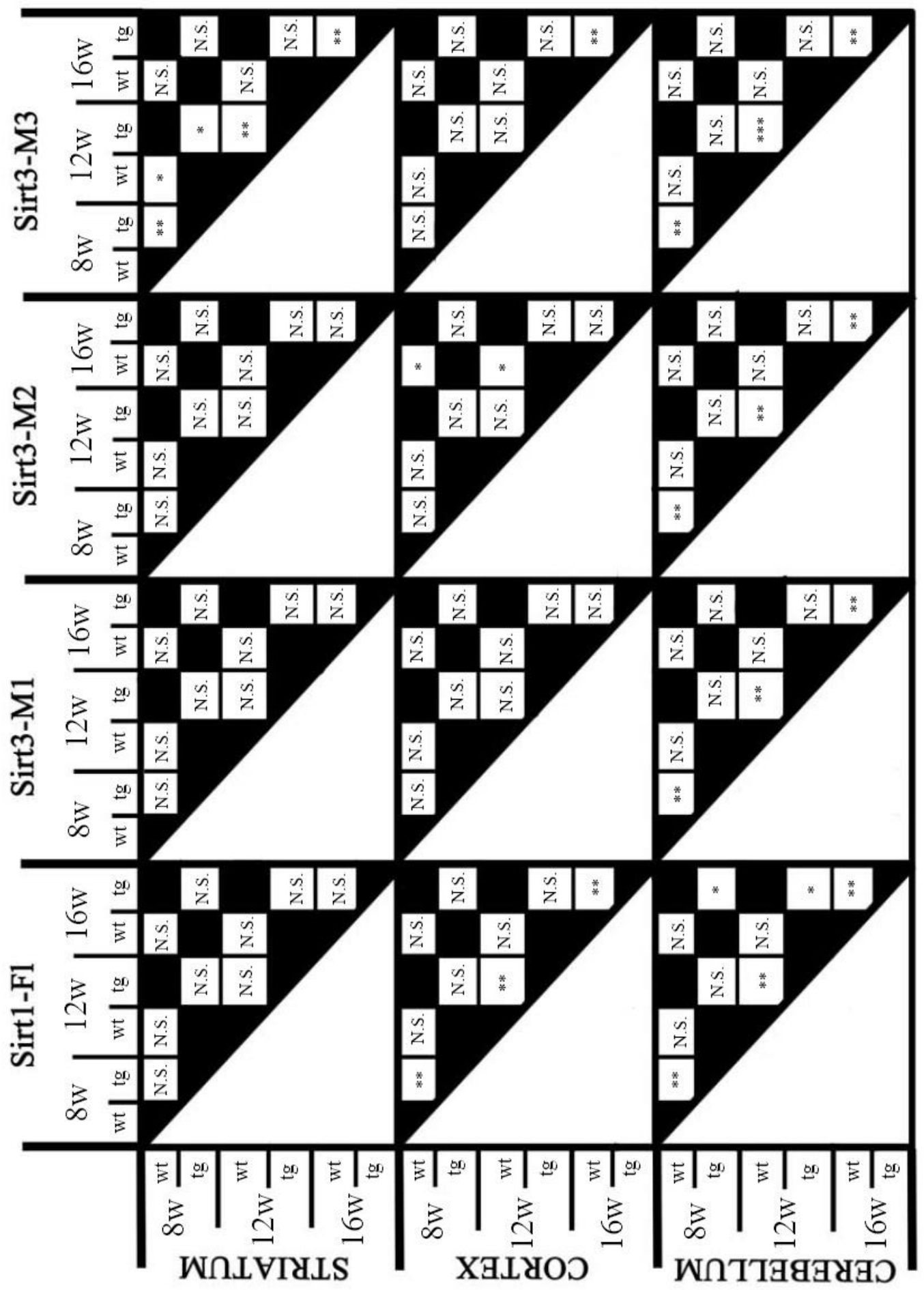

Figure 16. - This figure represents the effect of presence of transgene on the expression patterns (transgenic-wild-type comparisons) and on the time course (age-related alterations) and interaction as well. Regarding Sirt1 and Sirt3 expression, there were no significant interactions between the presence of the transgene and age. $* \mathrm{p}<0.05, * * \mathrm{p}<0.01, * * * \mathrm{p}<0.001 ; \mathrm{N} . \mathrm{S} .=$ not significant; $\operatorname{tg}=$ transgenic, $w t=$ wild-type, $w=$ weeks 


\section{V - DISCUSSION}

Mitochondrial dysfunction is one of the most relevant aspects of the pathomechanism of neurodegenerative disorders. Consequently, any attempt to maintain or restore the function of the mitochondrial system can be considered potentially neuroprotective. Many previously published scientific data suggest that two interdependent metabolic master regulator families, namely PGC- and Sirtuin-family could be relevant targets in this context. However, the detailed characterization of these systems has not yet been fully done. Therefore, the aim of the current researches was to investigate the function of these systems in detail (brain region and isoformspecific mRNA expression changes) in different environmental conditions (cold exposure, training exercise), and in toxin (MPTP) and transgenic models (N171-82Q) of PD and HD, respectively.

The effect of cooling on skeletal muscle and adipose tissue (especially on BAT) was extensively studied (Chang et al., 2012; Jankovic et al., 2015; Jokinen et al., 2017; Puigserver et al., 1998; Shi et al., 2005; Stancic et al., 2013; Wang and Tong, 2009; Yao et al., 2017; Zhang et al., 2009). Overall, the performed studies - although different experimental protocols (temperature, cooling period, different age and types of murine models) were used - suggest that important elements of the PGC- (e.g. FL-, NT-isoforms) and Sirtuin-systems (Sirt1, -2, -3, -6) are coldsensitive. There is only limited available data related to the alterations of these molecules in the central nervous system. Tritos et al. (2003) could not demonstrate any Pgc-1 $\alpha$ mRNA alteration in the adult (18-20-week-old, male) murine (C57BL/6J) brain after $4 \mathrm{~h}$ cold $\left(4^{\circ} \mathrm{C}\right)$ exposure. According to the results of the previous experiment, we could not demonstrate any alteration in the level of Pgc-1 $\alpha$ isoforms (FL-, NT-, CNS-, REF-Pgc-1 $\alpha$ ) in any brain area after cold exposure (either in $200 \mathrm{~min}$ or in $900 \mathrm{~min}$ protocols) as well. On the other hand, short (200 min) cold exposure elevated the level of cortical Sirt3-M1 mRNA level and decreased the cerebellar Sirt3-M3 level. Long exposure (900 min) resulted in a decline in cortical Sirt1, and striatal Sirt3-M1 levels. As an explanation, we presume that this cold-challenge regimen was not effective in decreasing the core body temperature sufficiently. We think that the early compensatory mechanisms in BAT and skeletal muscle may protect the brain against the effect of cold exposure. In conclusion, it seems that the Sirtuin-system may be more responsive to the cold stimulus than the PGC-system. 
The impact of exercise on PGC- and Sirtuin-systems was widely tested, however, the diversity of the models and the training strategies makes the interpretation and comparison of these results complicated. In the skeletal muscle there is a clear tendency of elevation regarding different Pgc-1 $\alpha$ isoforms (Costa et al., 2010; Huang et al., 2015; Lochmann et al., 2015; Shi et al., 2005; Tadaishi et al., 2011; Wen et al., 2014). The rate of increase depends on the applied training method and its intensity. It seems that during training with different intensities there is a complex program (including promoter) shifting in the gene expression regulation. Some members of the Sirtuin-family were also tested in order to find a correlation between training intensity, the age of animals and Sirtuin expression in the skeletal muscle (Bayod et al., 2012; Cheng et al., 2015; Garcia-Valles et al., 2013; Gusdon et al., 2017; Holloszy, 1997; Huang et al., 2015; Huang et al., 2016; Koltai et al., 2010; Shi et al., 2005; Steiner et al., 2011). In summary, there is a detectable increase in the level of Sirt 1 and Sirt6 after exercise. In contrast to the cold exposure, there are some, but mainly inconsistent data in the literature about the exercise-induced PGC- and Sirtuin alterations in the CNS. Steiner et al. (2011) tried to estimate the brain region-specific expression changes of these systems after 8 weeks of treadmill training (8-week-old ICR animals). There was an obvious elevation in both systems in many brain regions (e.g. cortex, hippocampus, frontal lobe). However, they did not attempt to detect the isoforms. In contrast to Steiner's findings, Gusdon et al. (2017) found no alteration in the PGC$1 \alpha$ and SIRT3 protein levels after 17-day-long training either in young or old mice (examined brain regions: cortex, striatum). Many other experiments were made with different protocols, however, the results remained controversial (Bayod et al., 2011; Cheng et al., 2015; Lezi et al., 2013, 2014; Marosi et al., 2012). Thus, during our research we tried to resolve the inconsistencies between the previously published studies and investigated the PGC-and Sirtuin isoforms in two different training protocols. The 5-day-long training period (short-term) did not cause alterations in Pgc-1 $\alpha$ transcripts in any brain regions. Contrarily, the 12-day-long (longterm) training period induced changes in all isoforms of the PGC-system in the cerebellum which is consistent with the results of Steiner et al. (2011). In the Sirtuin-system, the 5-daylong training also did not cause mRNA level alterations, but the long-term exercise resulted in the cerebellar elevation of Sirt3-M1 and Sirt3-M2 mRNA levels. These results suggest that very short-term exercise is unable to induce the PGC- and Sirtuin-systems. Contrarily, the 12-daylong training period induced changes in the cerebellum. We assume that the prominent cerebellar Pgc- and Sirtuin activation is connected to the development of synaptic plasticity between Purkinje cells. It leads to better motor coordination and integration of movements. Lucas et al. (2015) found in their research that there is a decrease in cell number and firing rate 
between the Purkinje cells in $P g c$ knockout mice, which finding further strengthens our assumption.

One of the most widely accepted models of PD are the MPTP (mitochondrial complex I inhibitor) toxin experiment. From the perspective of PGC-system, a previously published article suggests that the overexpression and the pharmacological stimulation (RESV) of $P g c-1 \alpha$ expression could be protective against MPTP toxicity (Breidert et al., 2002; Dehmer et al., 2004; Mudò et al., 2012). Furthermore, in the Pgc-1 $\alpha$ knockout mice, there is a pronounced susceptibility of dopaminergic neurons against this toxin (St-Pierre et al., 2006). However, it seems that the MPTP toxin induces only a short-term compensatory reaction in the PGC-system (Swanson et al., 2013). In our experiments, we tested the brain region and isoform specific reaction of PGC-system to MPTP toxin injection. We found that in the acute regimen, all the tested isoforms were elevated in all brain regions compared to the control animals. However, we could not detect this upregulation effect after 7 days of the last injection. We presume that MPTP is not highly selective, which explains the cerebellar activation as well. The effect of MPTP on Sirtuin-system is much less characterized. Two studies tested the potential neuroprotective effect of Sirtl overexpression against MPTP neurotoxicity (Kakefuda et al., 2009; Kitao et al., 2005). The conclusion of these experiments was that Sirtl overexpression did not alleviate the toxic effect of MPTP. There is a lack of scientific data about the effect of MPTP on other members of the Sirtuin family. However, it seems that in Sirt3 and Sirt5 null mice the MPTP-related toxic effect is more pronounced (Liu et al., 2015a, b). We found only a slight, but following normalization, not significant activation of cerebellar Sirt3 (mitochondrial) isoforms in the chronic experiment, which could be explained by the potential slower activation of the cerebellar Sirtuin-system elements.

Sirtuins are surely involved in the neurodegenerative process in HD, however, there are controversial results regarding their role (Naia and Rego, 2015; Neo and Tang, 2017; Reynolds et al., 2018; Tulino et al., 2016). Tulino et al. found a significant decrease in striatal Sirt1 mRNA expression from 4 to 9 weeks in the wt group, whereas cerebellar Sirt1 mRNA expression increased significantly by 9 and 14 weeks of age in the same control group in experiments with R6/2 mouse model of HD (MRN: 204) (Tulino et al., 2016). The presence of the transgene seemingly did not affect Sirt1 mRNA expression. Another research group (Reynolds et al.) measured Sirt1 mRNA levels in the whole-brain samples of 5, 8, 11- (MRN: 144) and 8-, 12-week-old (MRN: 182) R6/2 mice (Reynolds et al., 2018). In the 5-, 8- and 11week-old mice (MRN: 144) there was a significant increase in the mRNA levels of all tg groups. 
Aging did not affect the values and accordingly there was no significant interaction between age and the presence of the transgene. Regarding the only female cohort with 182 mean CAG repeat size, the significant increase could be observed only in the 8-week-old group (Reynolds et al., 2018). Due to the different CAG repeats, ages, brain regions and gender composition, the comparability of these results is limited. During the research, there was no significant differences between genders in respect of the above-mentioned aspects, and therefore, gender issues seemingly did not introduce bias into the studies of Tulino et al. and Reynolds et al. (Reynolds et al., 2018; Tulino et al., 2016). Similar to the study of Tulino et al., there was no detectable effect of the transgene in the striatum in any age groups, but a marked increase in Sirt1 expression was demonstrated in cortical and cerebellar samples of tg animals compared to wt controls in all age groups - similar to that was found by Reynolds et al. when applying whole brain samples. The magnitude of difference increased only by 16 weeks of age, and again, similarly to the latter study, no significant interaction was found between aging and the presence of the transgene. Aging-related increase in Sirt1 mRNA expression either in striatal or in cerebellar samples of wt mice, as found by Tulino et al., could not be confirmed by our study. Although data suggest that the induction of mitochondrially acting Sirt3 may be capable of exerting beneficial effects in a HD model (Fu et al., 2012), the expression pattern of Sirt3 mRNA isoforms has never been studied in any HD model. Similar to that was found in case of Sirt1-Fl, a remarkable increase of cerebellar expression of all Sirt3 isoforms could be observed in tg animals compared to wt controls in the current study. The striatal expression of Sirt-M3 in all age groups and the cortical expression of Sirt3-M3 by 16 weeks of age were found to be increased. However, the relative expression level of Sirt3-M3 mRNA is considerably lower compared to that of the other two isoforms. The expression level of cortical Sirt3-M2 in wt mice and striatal Sirt3-M3 in wt and tg mice decreased by 16 and 12 weeks of age, respectively. The pattern of expression changes in the cerebellum regarding any of the assessed SIRT subtypes and isoforms strongly resemble to the Pgc-1 $\alpha$ expression changes (either of its full length or N-terminal fragment) as we have shown in a previous study using the same animal model of HD (Török et al., 2015). The reason behind the same pattern may be that Sirtuins are upstream regulators of Pgc-1 $\alpha$ expression (Cui et al., 2006; Jodeiri Farshbaf and Ghaedi, 2017). Although the cerebellum is known not to be the primarily affected structure in HD, there is an increasing evidence of its involvement in the pathomechanism of the disorder (Samson and Claassen, 2017). A considerable loss of Purkinje cells was demonstrated in some HD patients with predominant motor symptoms (Singh-Bains et al., 2019), the extent of which may become more pronounced in patients with higher CAG repeat number (Hedjoudje et al., 2018). 
Furthermore, there is no clear relationship between the disease stage and the degree of Purkinje and granular cell loss, and the degree of cerebellar degeneration is quite variable (Gutekunst $e t$ al., 2002; Jeste et al., 1984; Rodda, 1985). The exact background of this variability, involving the sparing of alterations even in some human cases, is not known and needs further elucidation. Nevertheless, some studies proved cerebellar hypermetabolism in HD (Rees et al., 2014) with a presumed compensatory role for the dysfunction in the fronto-striato-thalamic motor circuit (Deckel, 1995; Squitieri et al., 2003). The significant elevations in cerebellar Pgc-1 $\alpha$ (Török et $a l ., 2015)$ and Sirt mRNA expressions in the N171-82Q HD model may be considered as an important part of this compensatory cerebellar hypermetabolism. Furthermore, the increased Sirt3 mRNA expression indicates the involvement of mitochondrial activation as well. The beneficial role of the SIRT-PGC-1 $\alpha$ axis in intact cerebellar functioning may be further supported by the finding that FL-Pgc- $1 \alpha$ knockout mice demonstrated reactive astrogliosis in cerebellar nuclei, whereas the striatum and cortex were almost totally spared (Szalárdy et al., 2013).

\section{VI - CONCLUSION}

Neurodegenerative diseases yield a growing health care problem, therefore, the identification of the potential novel aspects of the neurodegenerative mechanism could be a future target of drug design. From that reason we focused on the deep characterization of the PGC- and Sirtuin-system in different experimental protocols. Although the results of cold exposure were incongruent, but exercise training unequivocally could be an important potential activator of the cerebellar neuroprotective system including the PGC-Sirtuin axis. From the toxin experiment we concluded that the PGC-system is more sensitive to MPTP than the Sirtuins, however, not all isoforms were tested. In the N171-82Q model, in addition to the previously demonstrated activation of the cerebellar PGC-system, there was an obvious activation of cerebellar Sirtuin-system as well, which may emphasize the involvement of cerebellum in HD. However, in addition to the results detailed above, further studies are required to exactly clarify the role of the PGC- and Sirtuin-systems in the development of neurodegenerative processes. 


\section{VII - ACKNOWLEDGEMENTS}

I would like to express my gratitude to my supervisor Péter Klivényi, M.D., Ph.D., D.Sc. (Professor and Head of the Neurology Department, University of Szeged), who gave me a real opportunity to start a scientific work in the lab. I am grateful for his continued support in both the academic and personal areas of my life. I am especially grateful to my colleague Dénes Zádori, M.D., Ph.D. (Associate Professor at the Department of Neurology, University of Szeged) for his excellent scientific guidance and continuous support of my research activities. I had the opportunity to learn from him the value of thorough work and precision. I am much obliged to Rita Maszlag-Török, M.Sc., Ph.D. (Research fellow at the Department of Neurology, University of Szeged) for the possibility to become familiar with the laboratory methods and for her continuous support.

I am grateful to László Vécsei M.D., Ph.D., D.Sc. for the opportunity to work in the laboratory (Former Head of the Neurology Department, University of Szeged, Member of the Hungarian Academy of Sciences). Furthermore, I would like to say many thanks to all my colleagues, especially to Evelin Vágvölgyi-Sümegi, M.Sc., Fanni Annamária Boros, M.D., Gábor Veres, Pharm.D., Ph.D., László Szpisjak, M.D. for their help during my work.

Last but not least, I am grateful to my family, as without them this work would not have been possible. I thank my wife, Nóra for the time I got from them for the scientific work. I am grateful to my mother who raised and helped me during my studies. I dedicate this work to the memory of my father.

\section{VIII - REFERENCES}

Ahmad K, Baig MH, Mushtaq G, Kamal MA, Greig NH, Choi I (2017) Commonalities in biological pathways, genetics, and cellular mechanism between Alzheimer disease and other neurodegenerative diseases: an in silico-updated overview. Curr Alzheimer Res 14: 1190-97

Ahn BH, Kim HS, Song S, Lee IH, Liu J, Vassilopoulos A, Deng CX, Finkel T (2008) A role for the mitochondrial deacetylase Sirt3 in regulating energy homeostasis. Proc Natl Acad Sci USA 105: 14447-52 
Ajami M, Pazoki-Toroudi H, Amani H, Nabavi SF, Braidy N, Vacca RA, Atanasov AG, Mocan A, Nabavi SM (2017) Therapeutic role of sirtuins in neurodegenerative disease and their modulation by polyphenols. Neurosci Biobehav Rev 73: 39-47

Amat R, Planavila A, Chen SL, Iglesias R, Giralt M, Villarroya F (2009) SIRT1 controls the transcription of the peroxisome proliferator-activated receptor-gamma co-activator-1alpha (PGC-1alpha) gene in skeletal muscle through the PGC-1alpha autoregulatory loop and interaction with MyoD. J Biol Chem 284: 21872-80

Anekonda TS, Reddy PH (2006) Neuronal protection by sirtuins in Alzheimer's disease. J Neurochem 96: 305-313

Arun S, Liu L, Donmez G (2016) Mitochondrial biology and neurological diseases. Curr Neuropharmacol 14: 143-54

Austin S, St-Pierre J (2012) PGC1 $\alpha$ and mitochondrial metabolism - emerging concepts and relevance in ageing and neurodegenerative disorders. Journal of Cell Science 125: 4963-71

Baldo B, Gabery S, Soylu-Kucharz R, Cheong RY, Henningsen JB, Englund E, McLean C, Kirik D, Halliday G, Petersén $\AA$ (2019) SIRT1 is increased in affected brain regions and hypothalamic metabolic pathways are altered in Huntington disease. Neuropathol Appl Neurobiol 45: 361-79

Bao J, Lu Z, Joseph JJ, Carabenciov D, Dimond CC, Pang L, Samsel L, McCoy JP Jr, Leclerc J, Nguyen P, Gius D, Sack MN (2010) Characterization of the murine SIRT3 mitochondrial localization sequence and comparison of mitochondrial enrichment and deacetylase activity of long and short SIRT3 isoforms. J Cell Biochem 110: 238-247

Bayod S, Del Valle J, Canudas AM, Lalanza JF, Sanchez-Roige S, Camins A, Escorihuela RM, Pallàs M (2011) Long-term treadmill exercise induces neuroprotective molecular changes in rat brain. J Appl Physiol (1985) 111: 1380-90

Bayod S, Del Valle J, Lalanza JF, Sanchez-Roige S, de Luxán-Delgado B, Coto-Montes A, Canudas AM, Camins A, Escorihuela RM, Pallàs M (2012) Long-term physical exercise induces changes in sirtuin 1 pathway and oxidative parameters in adult rat tissues. Exp Gerontol 47: 925-935 
Belvirani M, Okudan N (2018) Exercise training protects against aging-induced cognitive dysfunction via activation of the hippocampal PGC- $1 \alpha /$ FNDC5/BDNF pathway. Neuromolecular Med 20: 386-400

Bose A, Beal MF (2016) Mitochondrial dysfunction in Parkinson's disease. J Neurochem 139(S1): 216-31

Blander G, Guarente L (2004) The Sir2 family of protein deacetylases. Annu Rev Biochem 73: 417-35

Breidert T, Callebert J, Heneka MT, Landreth G, Launay JM, Hirsch EC (2002) Protective action of the peroxisome proliferator-activated receptor-gamma agonist pioglitazone in a mouse model of Parkinson's disease. J Neurochem 82: 615-24

Brenmoehl J, Hoeflich A (2013) Dual control of mitochondrial biogenesis by sirtuin 1 and sirtuin 3. Mitochondrion 13: 755-61

Cabezas-Opazo FA, Vergara-Pulgar K, Pérez MJ, Jara C, Osorio-Fuentealba C, Quintanilla RA (2015) Mitochondrial dysfunction contributes to the pathogenesis of Alzheimer's disease. Oxid Med Cell Longev 2015: 509654

Cen Y, Youn DY, Sauve AA (2011) Advances in characterization of human sirtuin isoforms: chemistries, targets and therapeutic applications. Curr Med Chem 18: 1919-35

Chaturvedi RK, Adhihetty P, Shukla S, Hennessy T, Calingasan N, Yang L, Starkov A, Kiaei M, Cannella M, Sassone J, Ciammola A, Squitieri F, Beal MF (2009) Impaired PGC-1alpha function in muscle in Huntington's disease. Hum Mol Genet 18: 3048-65

Chang JS, Fernand V, Zhang Y, Shin J, Jun HJ, Joshi Y, Gettys TW (2012) NT-PGC-1alpha protein is sufficient to link beta3-adrenergic receptor activation to transcriptional and physiological components of adaptive thermogenesis. J Biol Chem 287: 9100-11

Cheng HC, Ulane CM, Burke RE (2010) Clinical progression in Parkinson disease and the neurobiology of axons. Ann Neurol 67: 715-25

Cheng A, Yang Y, Zhou Y, Maharana C, Lu D, Peng W, Liu Y, Wan R, Marosi K, Misiak M, Bohr VA, Mattson MP (2015) Mitochondrial SIRT3 mediates adaptive responses of neurons to exercise, and metabolic and excitatory challenges, Cell Metab 23: 128-142 
Chopra V, Quinti L, Kim J, Vollor L, Narayanan KL, Edgerly C, Cipicchio PM, Lauver MA, Choi SH, Silverman RB, Ferrante RJ, Hersch S, Kazantsev AG (2012) The sirtuin 2 inhibitor AK-7 is neuroprotective in Huntington's disease mouse models. Cell Rep 2: 1492-97

Clark J, Silvaggi JM, Kiselak T, Zheng K, Clore EL, Dai Y, Bass CE, Simon DK (2012) Pgc1alpha overexpression downregulates Pitx3 and increases susceptibility to MPTP toxicity associated with decreased BDNF. PLoS ONE 7: e48925

Cooper HM, Huang J-Y, Verdin E, Spelbrink JN (2009) A new splice variant of the mouse SIRT3 gene encodes the mitochondrial precursor protein. PLoS ONE 4:e4986

Costa Cdos S, Hammes TO, Rohden F, Margis R, Bortolotto JW, Padoin AV, Mottin CC, Guaragna RM (2010) SIRT1 transcription is decreased in visceral adipose tissue of morbidly obese patients with severe hepatic steatosis. Obes Surg 20: 633-39

Costa V, Scorrano L (2012) Shaping the role of mitochondria in the pathogenesis of Huntington's disease. EMBO J 31: 1853-64

Cui L, Jeong H, Borovecki F, Parkhurst CN, Tanese N, Krainc D (2006) Transcriptional repression of PGC-1alpha by mutant huntingtin leads to mitochondrial dysfunction and neurodegeneration. Cell 127: 59-69

Deckel AW (1995) Is Huntington's disease of cerebellar/brainstem origin? Lancet 345: 263-4

Dehmer T, Heneka MT, Sastre M, Dichgans J, Schulz JB (2004) Protection by pioglitazone in the MPTP model of Parkinson's disease correlates with I kappa B alpha induction and block of NF kappa B and iNOS activation. J Neurochem 88: 494-501

Deota S, Chattopadhyay T, Ramachandran D, Armstrong E, Camacho B, Maniyadath B, Fulzele A, Gonzalez-de-Peredo A, Denu JM, Kolthur-Seetharam U (2017) Identification of a tissue-restricted isoform of SIRT1 defines a regulatory domain that encodes specificity. Cell Rep 18: 3069-3077

Dominy JE Jr, Lee Y, Gerhart-Hines Z, Puigserver P (2009) Nutrient-dependent regulation of PGC-1alpha's acetylation state and metabolic function through the enzymatic activities of Sirt1/GCN5. Biochim Biophys Acta 1804: 1676-83

Donmez G (2012) The neurobiology of sirtuins and their role in neurodegeneration. Trends Pharmacol Sci 33: 494-501 
Donmez G, Arun A, Chung CY, McLean PJ, Lindquist S, Guarente L (2012) SIRT1 protects against alpha-synuclein aggregation by activating molecular chaperones. J Neurosci 32: 12432

Esterbauer H, Oberkofler H, Krempler F, Patsch W (1999) Human peroxisome proliferator activated receptor gamma coactivator 1 (PPARGC1) gene: cDNA sequence, genomic organization, chromosomal localization, and tissue expression. Genomics 62: 98-102

Fu J, Jin J, Cichewicz RH, Hageman SA, Ellis TK, Xiang L, Peng Q, Jiang M, Arbez N, Hotaling K, Ross CA, Duan W (2012) Trans-(-)-E-Viniferin increases mitochondrial sirtuin 3 (SIRT3), activates AMP-activated protein kinase (AMPK), and protects cells in models of Huntington Disease. J Biol Chem 287: 24460-72

Garcia-Valles R, Gomez-Cabrera MC, Rodriguez-Mañas L, Garcia-Garcia FJ, Diaz A, Noguera I, Olaso-Gonzalez G, Viña J (2013) Life-long spontaneous exercise does not prolong lifespan but improves health span in mice. Longev Healthspan 2: 14

Gerhart-Hines Z, Dominy JE Jr, Blättler SM, Jedrychowski MP, Banks AS, Lim JH, Chim H, Gygi SP, Puigserver P (2011) The cAMP/PKA pathway rapidly activates SIRT1 to promote fatty acid oxidation independently of changes in NAD(+). Mol Cell 44: 851-63

Gomes P, Fleming Outeiro T, Cavadas C (2015) Emerging Role of Sirtuin 2 in the Regulation of Mammalian Metabolism. Trends Pharmacol Sci 36: 756-768

Gusdon AM, Callio J, Distefano G, O'Doherty RM, Goodpaster BH, Coen PM, Chu CT (2017) Exercise increases mitochondrial complex I activity and DRP1 expression in the brains of aged mice. Exp Gerontol 90: 1-13

Gutekunst CA, Norflus F, Hersch SM (2002) The neuropathology of Huntington's disease. In: Bates GP, Harper PS, Jones AL (eds) Huntington's Disease, 4th edn. OUP, Oxford, pp 251-275

Hadem IKH, Majaw T, Kharbuli B, Sharma R (2019) Beneficial effects of dietary restriction in aging brain. J Chem Neuroanat 95: 123-133

Hajat C, Stein E (2018) The global burden of multiple chronic conditions: A narrative review. Prev Med Rep 12: 284-93

Hallows WC, Lee S, Denu JM (2006) Sirtuins deacetylate and activate mammalian acetyl-CoA synthetases. Proc Natl Acad Sci USA 103: 10230-10235 
Hathorn T, Snyder-Keller A, Messer A (2011) Nicotinamide improves motor deficits and upregulates PGC-1 $\alpha$ and BDNF gene expression in a mouse model of Huntington's disease. Neurobiol Dis 41: 43-50

Hedjoudje A, Nicolas G, Goldenberg A, Vanhulle C, Dumant-Forrest C, Deverrière G, Treguier P, Michelet I, Guyant-Maréchal L, Devys D, Gerardin E, Dacher JN, Vivier PH (2018) Morphological features in juvenile Huntington disease associated with cerebellar atrophy magnetic resonance imaging morphometric analysis. Pediatr Radiol 48: 1463-71

Hirsch EC, Jenner P, Przedborski S (2013) Pathogenesis of Parkinson's disease. Mov Disord 28:24-30

Holloszy JO (1997) Mortality rate and longevity of food-restricted exercising male rats: a reevaluation. J Appl Physiol 82: 399-403

Huang CC, Wang T, Tung YT, Lin WT (2016) Effect of exercise training on skeletal muscle SIRT1 and PGC-1 $\alpha$ expression levels in rats of different age. Int J Med Sci 13: 260-70

Huang LP, Yao M, Wang YL, Davie A, Zhou S (2015) A comparison of PGC-1 $\alpha$ mRNA and protein expression in response to 1-week endurance training on alternate days or 4 consecutive days. Appl Physiol Nutr Metab 40: 1210-13

Jankovic A, Golic I, Markelic M, Stancic A, Otasevic V, Buzadzic B, Korac A, Korac B (2015) Two key temporally distinguishable molecular and cellular components of white adipose tissue browning during cold acclimation. J Physiol 593: 3267-80

Jeong H, Cohen DE, Cui L, Supinski A, Savas JN, Mazzulli JR, Yates JR, Bordone L, Guarente L, Krainc D (2011) Sirt1 mediates neuroprotection from mutant huntingtin by activation of the TORC1 and CREB transcriptional pathway. Nat Med 18: 159-65

Jęśko H, Wencel P, Strosznajder RP, Strosznajder JB (2017) Sirtuins and Their Roles in Brain Aging and Neurodegenerative Disorders. Neurochem Res 42: 876-90

Jeste DV, Barban L, Parisi J (1984) Reduced Purkinje cell density in Huntington's disease. Exp Neurol 85: 78-86

Jiang M, Wang J, Fu J, Du L, Jeong H, West T, Xiang L, Peng Q, Hou Z, Cai H, Seredenina T, Arbez N, Zhu S, Sommers K, Qian J, Zhang J, Mori S, Yang XW, Tamashiro KL, Aja S, Moran TH, Luthi-Carter R, Martin B, Maudsley S, Mattson MP, Cichewicz RH, Ross CA, Holtzman 
DM, Krainc D, Duan W (2011) Neuroprotective role of Sirt1 in mammalian models of Huntington's disease through activation of multiple Sirt1 targets. Nat Med 18: 153-58

Jin L, Galonek H, Israelian K, Choy W, Morrison M, Xia Y, Wang X, Xu Y, Yang Y, Smith JJ, Hoffmann E, Carney DP, Perni RB, Jirousek MR, Bemis JE, Milne JC, Sinclair DA, Westphal CH (2009) Biochemical characterization, localization, and tissue distribution of the longer form of mouse SIRT3. Protein Sci 18: 514-525

Jodeiri Farshbaf M, Ghaedi K (2017) Huntington's disease and mitochondria. Neurotox Res 32: 518-29

Johri A, Chandra A, Flint Beal M (2013) PGC-1 $\alpha$, mitochondrial dysfunction, and Huntington's disease. Free Radic Biol Med 62: 37-46

Johri A, Starkov AA, Chandra A, Hennessey T, Sharma A, Orobello S, Squitieri F, Yang L, Beal MF (2011) Truncated peroxisome proliferator-activated receptor- $\gamma$ coactivator $1 \alpha$ splice variant is severely altered in Huntington's disease. Neurodegener Dis 8: 496-503

Jokinen R, Pirnes-Karhu S, Pietiläinen KH, Pirinen E (2017) Adipose tissue NAD+homeostasis, sirtuins and poly(ADP-ribose) polymerases -important players in mitochondrial metabolism and metabolic health. Redox Biol 12: 246-63

Kakefuda K, Fujita Y, Oyagi A, Hyakkoku K, Kojima T, Umemura K, Tsuruma K, Shimazawa M, Ito M, Nozawa Y, Hara H (2009) Sirtuin 1 overexpression mice show a reference memory deficit, but not neuroprotection. Biochem Biophys Res Commun 387: 784-8

Kelly G (2010a) A review of the sirtuin system, its clinical implications, and the potential role of dietary activators like resveratrol: part 1. Altern Med Rev 15: 245-263

Kelly G (2010b) A review of the sirtuin system, its clinical implications, and the potential role of dietary activators like resveratrol: part 2. Altern Med Rev 15: 313-328

Kitao Y, Ageta-Ishihara N, Takahashi R, Kinoshita M, Hori1 O (2015) Transgenic supplementation of SIRT1 fails to alleviate acute loss of nigrostriatal dopamine neurons and gliosis in a mouse model of MPTP-induced parkinsonism. F1000Research 4: 130

Koltai E, Szabo Z, Atalay M, Boldogh I, Naito H, Goto S, Nyakas C, Radak Z (2010) Exercise alters SIRT1, SIRT6, NAD and NAMPT levels in skeletal muscle of aged rats. Mech Ageing Dev 131: 21-8 
Kong X, Wang R, Xue Y, Liu X, Zhang H, Chen Y, Fang F, Chang Y (2010) Sirtuin 3, a new target of PGC-1alpha, plays an important role in the suppression of ROS and mitochondrial biogenesis. PLoS One 5: e11707

Koutsilieri E, Riederer P (2007) Excitotoxicity and new antiglutamatergic strategies in Parkinson's disease and Alzheimer's disease. Parkinsonism Relat Disord 13: S329-31

La Spada AR (2012) Finding a sirtuin truth in Huntington's disease. Nat Med 18: 24-26

Langston JW (2017) The MPTP story. J Parkinsons Dis 7: S11-19

Lee IH, Cao L, Mostoslavsky R, Lombard DB, Liu J, Bruns NE, Tsokos M, Alt FW, Finkel T. (2008) A role for the NAD-dependent deacetylase Sirt1 in the regulation of autophagy. Proc Natl Acad Sci USA 105: 3374-79

Leone TC, Lehman JJ, Finck BN, Schaeffer PJ, Wende AR, Boudina S, Courtois M, Wozniak DF, Sambandam N, Bernal-Mizrachi C, Chen Z, Holloszy JO, Medeiros DM, Schmidt RE, Saffitz JE, Abel ED, Semenkovich CF, Kelly DP (2005) PGC-1alpha deficiency causes multisystem energy metabolic derangements: muscle dysfunction, abnormal weight control and hepatic steatosis. PLoS Biol 3: e101

Lez E, Lu J, Burns JM, Swerdlow RH (2013) Effect of exercise on mouse liver and brain bioenergetic infrastructures. Exp Physiol 98: 207-19

Lezi E, Burns JM, Swerdlow RH (2014) Effect of high-intensity exercise on aged mouse brain mitochondria, neurogenesis, and inflammation. Neurobiol Aging 35: 2574-83

Lezi E, Swerdlow RH (2012) Mitochondria in neurodegeneration. Adv Exp Med Biol 942: 26986

Liang H, Ward WF (2006) PGC-1alpha: a key regulator of energy metabolism. Adv Physiol Educ 30: 145-51

Lin J, Handschin C, Spiegelman BM (2005) Metabolic control through the PGC-1 family of transcription coactivators. Cell Metab 1: 361-70

Lin J, Wu PH, Tarr PT, Lindenberg KS, St-Pierre J, Zhang CY, Mootha VK, Jäger S, Vianna CR, Reznick RM, Cui L, Manieri M, Donovan MX, Wu Z, Cooper MP, Fan MC, Rohas LM, Zavacki AM, Cinti S, Shulman GI, Lowell BB, Krainc D, Spiegelman BM (2004) Defects in 
adaptive energy metabolism with CNS-linked hyperactivity in PGC-1alpha null mice. Cell 119: 121-35

Lin J, Wu PH, Tarr PT, Zhang CY, Wu Z, Boss O, Michael LF, Puigserver P, Isotani E, Olson EN, Lowell BB, Bassel-Duby R, Spiegelman BM (2002) Transcriptional co-activator PGC-1 alpha drives the formation of slow-twitch muscle fibres. Nature 418: 797-801

Livak KJ, Schmittgen TD (20019 Analysis of relative gene expression data using realtime quantitative PCR and the 2(-Delta DeltaC(T)) Method. Methods 25: 402-8

Lochmann TL, Thomas RR, Bennett JP Jr, Taylor SM (2015) Epigenetic modifications of the PGC-1 $\alpha$ promoter during exercise induced expression in mice. PLoS One 10: e0129647

Liu L, Peritore C, Ginsberg J, Kayhan M, Donmez G (2015a) SIRT3 attenuates MPTP-induced nigrostriatal degeneration via enhancing mitochondrial antioxidant capacity. Neurochem Res 40: $600-8$

Liu L, Peritore C, Ginsberg J, Shih J, Arun S, Donmez G (2015b) Protective role of SIRT5 against motor deficit and dopaminergic degeneration in MPTP-induced mice model of Parkinson's disease. Behav Brain Res 281: 215-21

Lucas EK, Reid CS, McMeekin LJ, Dougherty SE, Floyd CL, Cowell RM (2015) Cerebellar transcriptional alterations with Purkinje cell dysfunction and loss in mice lacking PGC-1 $\alpha$. Front Cell Neurosci 8: 441

Lynch CJ, Shah ZH, Allison SJ, Ahmed SU, Ford J, Warnock LJ, Li H, Serrano M, Milner J (2010) SIRT1 undergoes alternative splicing in a novel auto-regulatory loop with p53. PLoS One 5: e13502

Majláth Z, Toldi J, Fülöp F, Vécsei L (2016) Excitotoxic mechanisms in non-motor dysfunctions and levodopa- induced dyskinesia in Parkinson's disease: the role of the interaction between the dopaminergic and the kynurenine system. Curr Med Chem 23: 874-83 Mandel S, Grünblatt E, Riederer P, Gerlach M, Levites Y, Youdim MB (2003) Neuroprotective strategies in Parkinson's disease: an update on progress. CNS Drugs 17: 729-62

Marosi K, Bori Z, Hart N, Sárga L, Koltai E, Radák Z, Nyakas C (2012) Long-term exercise treatment reduces oxidative stress in the hippocampus of aging rats. Neuroscience 226: 21-8 
Martínez-Redondo V, Pettersson AT, Ruas JL (2015) The hitchhiker's guide to PGC-1 $\alpha$ isoform structure and biological functions. Diabetologia 58: 1969-77

Maxwell MM, Tomkinson EM, Nobles J, Wizeman JW, Amore AM, Quinti L, Chopra V, Hersch SM, Kazantsev AG (2011) The Sirtuin 2 microtubule deacetylase is an abundant neuronal protein that accumulates in the aging CNS. Hum Mol Genet 20: 3986-96

McGill JK, Beal MF (2006) PGC-1alpha, a new therapeutic target in Huntington's disease? Cell 127: $465-8$

Michishita E, Park JY, Burneskis JM, Barrett JC, Horikawa I (2005) Evolutionarily conserved and nonconserved cellular localizations and functions of human SIRT proteins. Mol Biol Cell 16: $4623-4635$

Mudò G, Mäkelä J, Di Liberto V, Tselykh TV, Olivieri M, Piepponen P, Eriksson O, Mälkiä A, Bonomo A, Kairisalo M, Aguirre JA, Korhonen L, Belluardo N, Lindholm D (2012) Transgenic expression and activation of PGC-1 $\alpha$ protect dopaminergic neurons in the MPTP mouse model of Parkinson's disease. Cell Mol Life Sci 69: 1153-65

Naia L, Rego AC (2015) Sirtuins: double players in Huntington's disease. Biochim Biophys Acta 1852: 2183-94

Nemoto S, Fergusson MM, Finkel T (2005) SIRT1 functionally interacts with the metabolic regulator and transcriptional coactivator PGC-1 \{alpha\}. J Biol Chem 280: 16456-60

Neo SH, Tang BL (2017) Sirtuins as modifiers of Huntington's disease (HD) pathology. Prog Mol Biol Transl 154: 105-145

Nogueiras R, Habegger KM, Chaudhary N, Finan B, Banks AS, Dietrich MO, Horvath TL, Sinclair DA, Pfluger PT, Tschöp MH (2012) Sirtuin 1 and sirtuin 3: physiological modulators of metabolism. Physiol Rev 92: 1479-514

Nunomura A, Moreira PI, Lee HG, Zhu X, Castellani RJ, Smith MA et al (2007) Neuronal death and survival under oxidative stress in Alzheimer and Parkinson diseases. CNS Neurol Disord Drug Targets 6: 411-423

Paraíso AF, Mendes KL, Santos SH (2013) Brain activation of SIRT1: role in neuropathology. Mol Neurobiol 48: 681-9 
Parker JA, Arango M, Abderrahmane S, Lambert E, Tourette C, Catoire H, Néri C (2005) Resveratrol rescues mutant polyglutamine cytotoxicity in nematode and mammalian neurons. Nat Genet 37: 349-50

Pallàs M, Verdaguer E, Tajes M, Gutierrez-Cuesta J, Camins A (2008) Modulation of sirtuins: new targets for antiageing. Recent Pat CNS Drug Discov 3: 61-9

Postuma RB, Berg D, Stern M, Poewe W, Olanow CW, Oertel W, Obeso J, Marek K, Litvan I, Lang AE, Halliday G, Goetz CG, Gasser T, Dubois B, Chan P, Bloem BR, Adler CH, Deuschl G (2015) MDS clinical diagnostic criteria for Parkinson's disease. Mov Disord 30: 1591-601

Puigserver P, Wu Z, Park CW, Graves R, Wright M, Spiegelman BM (1998) A cold-inducible coactivator of nuclear receptors linked to adaptive thermogenesis. Cell 92: 829-39

Puigserver P, Spiegelman BM (2003) Peroxisome proliferator-activated receptor-gamma coactivator 1 alpha (PGC-1 alpha): transcriptional coactivator and metabolic regulator. Endocr Rev 24: 78-90

Qiu X, Brown K, Hirschey MD, Verdin E, Chen D (2010) Calorie restriction reduces oxidative stress by SIRT3-mediated SOD2 activation. Cell Metab 12: 662-7

Rasouri S, Lagouge M, Auwerx J (2007) SIRT1/PGC-1: a neuroprotective axis? Med Sci (Paris) 23: 840-44

Rawlins MD, Wexler NS, Wexler AR, Tabrizi SJ, Douglas I, Evans SJ, Smeeth L (2016) The Prevalence of Huntington's Disease. Neuroepidemiology 46: 144-53

Rees EM, Farmer R, Cole JH, Haider S, Durr A, Landwehrmeyer B (2014) Cerebellar abnormalities in Huntington's disease: a role in motor and psychiatric impairment? Mov Disord 29: $1648-54$

Relja M (2004) Pathophysiology and classification of neurodegenerative diseases. EJIFCC 15: 97-99

Reynolds RH, Petersen MH, Willert CW, Heinrich M, Nymann N, Dall M, Treebak JT, Björkqvist M, Silahtaroglu A, Hasholt L, Nørremølle A (2018) Perturbations in the p53/miR34a/SIRT1 pathway in the R6/2 Huntington's disease model. Mol Cell Neurosci 88: 118-29

Rodda RA (1981) Cerebellar atrophy in Huntington's disease. J Neurol Sci 50: 147-57 
Rodgers JT, Lerin C, Haas W, Gygi SP, Spiegelman BM, Puigserver P (2005) Nutrient control of glucose homeostasis through a complex of PGC-1alpha and SIRT1. Nature 434: 113-8

Róna-Vörös K, Weydt P (2010) The role of PGC-1 $\alpha$ in the pathogenesis of neurodegenerative disorders. Curr Drug Targets 11: 1262-69

Salamon A, Zádori D, Szpisjak L, Klivényi P, Vécsei L (2019) Neuroprotection in Parkinson's disease: facts and hopes. J Neural Transm (Vienna) https://doi.org/10.1007/s00702-019-021158

Sambamoorthi U, Tan X, Deb A (2015) Multiple chronic conditions and healthcare costs among adults. Expert Rev Pharmacoecon Outcomes Res 15: 823-32

Samson M, Claassen DO (2017) Neurodegeneration and the cerebellum. Neurodegener Dis 17: $155-65$

Schiedel M, Robaa D, Rumpf T, Sippl W, Jung M (2018) The current state of NAD+-dependent histone deacetylases (Sirtuins) as novel therapeutic targets. Med Res Rev 38: 147-200

Schulte J, Littleton JT (2011) The biological function of the Huntingtin protein and its relevance to Huntington's disease pathology. Curr Trends Neurol 5: 65-78

She DT, Jo DG, Arumugam TV (2017) Emerging roles of Sirtuins in ischemic stroke. Transl Stroke Res 8: 405-423

Shi P, Gal J, Kwinter DM, Liu X, Zhu H (2010) Mitochondrial dysfunction in amyotrophic lateral sclerosis. Biochim Biophys Acta 1802: 45-51

Shi T, Wang F, Stieren E, Tong Q (2005) SIRT3, a mitochondrial sirtuin deacetylase, regulates mitochondrial function and thermogenesis in brown adipocytes. J Biol Chem 280: 13560-7

Singh-Bains MK, Mehrabi NF, Sehji T, Austria MDR, Tan AYS, Tippett LJ et al (2019) Cerebellar degeneration correlates with motor symptoms in Huntington disease. Ann Neurol 85: 396-405

Snowden MB, Bowen JD, Hughes J, Larson EB (1995) Study of Alzheimer's dementia patients with parkinsonian features. J Geriatr Psychiatry Neurol 8: 154-58

Soyal SM, Felder TK, Auer S, Hahne P, Oberkofler H, Witting A, Paulmichl M, Landwehrmeyer GB, Weydt P, Patsch W, European Huntington Disease Network (2012) A 
greatly extended PPARGC1A genomic locus encodes several new brain-specific isoforms and influences Huntington disease age of onset. Hum Mol Genet 21: 3461-73

Squitieri F, Pustorino G, Cannella M, Toscano A, Maglione V, Morgante L (2003) Highly disabling cerebellar presentation in Huntington disease. Eur J Neurol 10: 443-4

St-Pierre J, Drori S, Uldry M, Silvaggi JM, Rhee J, Jäger S, Handschin C, Zheng K, Lin J, Yang W, Simon DK, Bachoo R, Spiegelman BM (2006) Suppression of reactive oxygen species and neurodegeneration by the PGC-1 transcriptional coactivators. Cell 127: 397-408

Stancic A, Buzadzic B, Korac A, Otasevic V, Jankovic A, Vucetic M, Markelic M, Velickovic K, Golic I, Korac B (2013) Regulatory role of PGC-1 $\alpha /$ PPAR signaling in skeletal muscle metabolic recruitment during cold acclimation. J Exp Biol 216: 4233-41

Steiner JL, Murphy EA, McClellan JL, Carmichael MD, Davis JM (2011) Exercise training increases mitochondrial biogenesis in the brain. J Appl Physiol (1985) 111: 1066-71

Sun AY, Wang Q, Simonyi A, Sun GY (2010) Resveratrol as a therapeutic agent for neurodegenerative diseases. Mol Neurobiol 41: 375-83

Swanson CR, Du E, Johnson DA, Johnson JA, Emborg ME (2013) Neuroprotective properties of a novel non-thiazolidinedione partial PPAR gamma agonist against MPTP. PPAR Res 2013: 582809

Szalardy L, Zadori D, Plangar I, Vecsei L, Weydt P, Ludolph AC (2013) Neuropathology of partial PGC-1 $\alpha$ deficiency recapitulates features of mitochondrial encephalopathies but not of neurodegenerative diseases. Neurodegener Dis 12: 177-88

Tabrizi SJ, Leavitt BR, Landwehrmeyer GB, Wild EJ, Saft C, Barker RA (2019) Targeting huntingtin expression in patients with Huntington's disease. N Engl J Med 380: 2307-16

Tadaishi M, Miura S, Kai Y, Kawasaki E, Koshinaka K, Kawanaka K, Nagata J, Oishi Y, Ezaki $\mathrm{O}$ (2011) Effect of exercise intensity and AICAR on isoform-specific expressions of murine skeletal muscle PGC-1 $\alpha$ mRNA: a role of $\beta_{2}$-adrenergic receptor activation. Am J Physiol Endocrinol Metab 300: 341-349

Török, R, Kónya JA, Zádori D, Veres G, Szalárdy L, Vécsei L, Klivényi P (2015) mRNA expression levels of PGC-1 $\alpha$ in a transgenic and a toxin model of Huntington's disease. Cell Mol Neurobiol 35: 293-301 
Tritos NA, Mastaitis JW, Kokkotou EG, Puigserver P, Spiegelman BM, Maratos-Flier E (2003) Characterization of the peroxisome proliferator activated receptor coactivator 1 alpha (PGC 1alpha) expression in the murine brain. Brain Res 961: 255-60

Tulino R, Benjamin AC, Jolinon N, Smith DL, Chini EN, Carnemolla A, Bates GP (2016) SIRT1 activity is linked to its brain region-specific phosphorylation and is impaired in Huntington's disease mice. PLoS One 11: e0145425

Vonsattel JP, Myers RH, Stevens TJ, Ferrante RJ, Bird ED, Richardson EP Jr (1985) Neuropathological classification of Huntington's disease. J Neuropathol Exp Neurol 44: 55977

Wang Y, Chen C, Huang W, Huang M, Wang J, Chen X, Ye Q (2019) Beneficial effects of PGC-1 $\alpha$ in the substantia nigra of a mouse model of MPTP-induced dopaminergic neurotoxicity. Aging (Albany NY) 11: 8937-50

Wang F, Tong Q (2009) SIRT2 suppresses adipocyte differentiation by deacetylating FOXO1 and enhancing FOXO1's repressive interaction with PPAR gamma. Mol Biol Cell 20: 801-8

Wareski P, Vaarmann A, Choubey V, Safiulina D, Liiv J, Kuum M, Kaasik A (2009) PGC1alpha and PGC-1beta regulate mitochondrial density in neurons. J Biol Chem 284: 21379-85

Wen X, Wu J, Chang JS, Zhang P, Wang J, Zhang Y, Gettys TW, Zhang Y (2014) Effect of exercise intensity on isoform-specific expressions of NT-PGC-1 $\alpha$ mRNA in mouse skeletal muscle. Biomed Res Int 2014: 402175

Weydt P, Pineda VV, Torrence AE, Libby RT, Satterfield TF, Lazarowski ER, Gilbert ML, Morton GJ, Bammler TK, Strand AD, Cui L, Beyer RP, Easley CN, Smith AC, Krainc D, Luquet S, Sweet IR, Schwartz MW, La Spada AR (2006) Thermoregulatory and metabolic defects in Huntington's disease transgenic mice implicate PGC-1alpha in Huntington's disease neurodegeneration. Cell Metab 4: 349-62

Yang Y, Hubbard BP, Sinclair DA, Tong Q (2010) Characterization of murine SIRT3 transcript variants and corresponding protein products. J Cell Biochem 111: 1051-8

Yao L, Cui X, Chen Q, Yang X, Fang F, Zhang J, Liu G, Jin W, Chang Y (2017) Cold inducible SIRT6 regulates thermogenesis of brown and beige fat. Cell Rep 20: 641-54 
Zakhary SM, Ayubcha D, Dileo JN, Jose R, Leheste JR, Horowitz JM, Torres G (2010) Distribution analysis of deacetylase SIRT1 in rodent and human nervous systems. Anat Rec (Hoboken) 293: 1024-32

Zhang A, Wang H, Qin X, Pang S, Yan B (2012) Genetic analysis of SIRT1 gene promoter in sporadic Parkinson's disease. Biochem Biophys Res Commun 422: 693-96

Zhang Y, Huypens P, Adamson AW, Chang JS, Henagan TM, Boudreau A, Lenard NR, Burk D, Klein J, Perwitz N, Shin J, Fasshauer M, Kralli A, Gettys TW (2009) Alternative mRNA splicing produces a novel biologically active short isoform of PGC-1alpha. J Biol Chem 284: 32813-26

Zhang F, Wang S, Gan L, Vosler PS, Gao Y, Zigmond MJ, Chen J (2011) Protective effects and mechanisms of sirtuins in the nervous system. Prog Neurobiol 95: 373-95

Zhong L, Mostoslavsky R (2011) Fine tuning our cellular factories: sirtuins in mitochondrial biology. Cell Metab 13: 621-26

Zádori D, Klivényi P, Plangár I, Toldi J, Vécsei L (2011) Endogenous neuroprotection in chronic neurodegenerative disorders: with particular regard to the kynurenines. J Cell Mol Med 15: $701-17$

Zádori D, Klivényi P, Szalárdy L, Fülöp F, Toldi J, Vécsei L (2012) Mitochondrial disturbances, excitotoxicity, neuroinflammation and kynurenines: novel therapeutic strategies for neurodegenerative disorders. J Neurol Sci 322: 187-91

Zádori D, Nyiri G, Szonyi A, Szatmári I, Fülöp F, Toldi J, Freund TF, Vécsei L, Klivényi P (2011) Neuroprotective effects of a novel kynurenic acid analogue in a transgenic mouse model of Huntington's disease. J Neural Transm (Vienna) 118: 865-75

Zádori D, Szalárdy L, Toldi J, Fülöp F, Klivényi P, Vécsei L (2013) Some molecular mechanisms of dopaminergic and glutamatergic dysfunctioning in Parkinson's disease. J Neural Transm 120: 673-81 


\title{
Cerebellar Predominant Increase in mRNA Expression Levels of Sirt1 and Sirt3 Isoforms in a Transgenic Mouse Model of Huntington's Disease
}

\author{
Andras Salamon ${ }^{1}$. Rita Maszlag-Török ${ }^{1}$ - Gábor Veres ${ }^{1,2}$ • Fanni Annamária Boros ${ }^{1}$ - Evelin Vágvölgyi-Sümegi ${ }^{1}$. \\ Anett Somogyi ${ }^{1} \cdot$ László Vécsei $^{1,2}$ - Péter Klivényi ${ }^{1}$ - Dénes Zádori ${ }^{1}[$
}

Received: 13 January 2020 / Revised: 1 May 2020 / Accepted: 4 June 2020

(c) The Author(s) 2020

\begin{abstract}
The potential role of Sirt1 and Sirt2 subtypes of Sirtuins (class III NAD ${ }^{+}$-dependent deacetylases) in the pathogenesis of Huntington's disease (HD) has been extensively studied yielding some controversial results. However, data regarding the involvement of Sirt3 and their variants in HD are considerably limited. The aim of this study was to assess the expression pattern of Sirt1 and three Sirt3 mRNA isoforms (Sirt3-M1/2/3) in the striatum, cortex and cerebellum in respect of the effect of gender, age and the presence of the transgene using the N171-82Q transgenic mouse model of HD. Striatal, cortical and cerebellar Sirt1-Fl and Sirt3-M1/2/3 mRNA levels were measured in 8, 12 and 16 weeks old N171-82Q transgenic mice and in their wild-type littermates. Regarding the striatum and cortex, the presence of the transgene resulted in a significant increase in Sirt3-M3 and Sirt1 mRNA levels, respectively, whereas in case of the cerebellum the transgene resulted in increased expression of all the assessed subtypes and isoforms. Aging exerted minor influence on Sirt mRNA expression levels, both in transgene carriers and in their wild-type littermates, and there was no interaction between the presence of the transgene and aging. Furthermore, there was no difference between genders. The unequivocal cerebellar Sirtuin activation with presumed compensatory role suggests that the cerebellum might be another key player in HD in addition to the most severely affected striatum. The mitochondrially acting Sirt3 may serve as an interesting novel therapeutic target in this deleterious condition.
\end{abstract}

Keywords Huntington's disease $\cdot$ Transgenic $\cdot$ Sirt $1 \cdot$ Sirt3 $\cdot$ Sirtuin $\cdot$ Brain

\section{Introduction}

Huntington's disease (HD) is an autosomal dominant neurodegenerative disease [66]. HD is caused by expansion of CAG repeats in the IT15 gene encoding Huntington protein $(\mathrm{Htt})$ which has an important role in the maintenance of cellular energy metabolism and mitochondrial function [50]. Previous works demonstrated that mutant Huntington protein $(\mathrm{mHtt})$ inhibits the function of a key metabolic master regulator, namely peroxisome proliferator-activated

Dénes Zádori

zadori.denes@med.u-szeged.hu

1 Department of Neurology, Interdisciplinary Excellence Center, Faculty of Medicine, Albert Szent-Györgyi Clinical Center, University of Szeged, Semmelweis u. 6, Szeged 6725, Hungary

2 MTA-SZTE Neuroscience Research Group of the Hungarian Academy of Sciences, Budapest, Hungary receptor-gamma coactivator $1 \alpha$ (PGC- $1 \alpha)$, which, amongst others, has an essential role in mitochondrial biogenesis $[8$, 25].

Sirtuins are class III NAD ${ }^{+}$-dependent deacetylases [38]. Currently there are seven identified mammalian Sirtuin subtypes (SIRT1-7), which are localized in different cellular compartments (nuclear: SIRT1 (the mammalian orthologue of the yeast Silent information regulator 2 protein (Sir2)), $-6,-7$; mitochondrial: SIRT3, $-4,-5$; cytoplasmatic: SIRT2) [36]. In addition to the above-detailed subtypes, alternative splicing results in further isoforms of Sirtuins [31, 67]. Several molecular targets of Sirtuins, including the abovementioned PGC- $1 \alpha$, were identified as participants of the regulation of energy metabolism, circadian rhythm, stress response, apoptosis and aging [38]. The association between SIRTs and neurodegenerative disorders, including HD, has been widely studied using these models $[2,22,24,27,36$, 56]. Calorie restriction is capable of increasing SIRT1 protein level in the brain, liver, hearth and white adipose tissue 
of mice [39], and also increases the lifespan in the N17182Q transgenic ( $\mathrm{tg}$ ) mouse model of HD [11]. In contrast to these findings, exercise, which induces the expression of Sirt3-M1 and -M2 isoforms [47], did not elongate the lifespan in the same mouse model of HD [43]. Regarding Sirt1 mRNA and SIRT1 protein expression changes in HD the results are somewhat inconsistent: SIRT1 protein levels were found to be reduced in human brain tissue and in the R6/1 transgenic mouse model of HD as well [18, 41]. Tulino et al. found that SIRT1 activity becomes reduced in R6/2 (with a mean CAG repeat number (MRN) of 204) and HdhQ150 (MRN: 165) mice, in the background of which they hypothesized altered phosphorylation status of SIRT1 via an 5' AMP-activated protein kinase $\alpha 1$-related mechanism in the striatum and cerebellum [61]. Although the presence of the transgene did not affect either Sirt1 mRNA, or SIRT1 protein expression in the striatum of R6/2 mice, there was a significant decrease in mRNA expression from 4 to 9 weeks in wild-type (wt) animals [61]. In contrast to these findings, cerebellar Sirt1 mRNA expression increased significantly by 9 and 14 weeks of age in R6/2 mice, whereas SIRT1 protein levels significantly decreased predominantly in wt mice by 14 weeks of age. In $H d h \mathrm{Q} 150$ and wt mice the SIRT1 protein level did not show any alteration either in the 2 or in the 22 months old animals [61]. A later wholebrain study reported the elevation of Sirt1 mRNA level in 5, 8 and 11 weeks old R6/2 mice (MRN: 144). However, up-regulation of Sirt 1 was only observed in their 8 , but not in 12 weeks old counterparts possessing a mean CAG repeat size of 182 [45]. Regarding SIRT1 protein expression, there was a consistent elevation in tg mice with 182 mean CAG repeats in both ages, and when examined at 12 weeks of age, without gender differences [45]. Besides the somewhat inconsistent findings obtained from the abovementioned studies, the experiments influencing the expression of SIRT1 or of its orthologues from a therapeutic point of view yielded more controversial results. Parker et al. were the first who demonstrated that Sir2 overexpression and resveratrol (RESV) treatment (one of the most important non-selective Sirtuin inducer) could delay the development of neuronal dysfunction in a Caenorhabditis elegans model of $\mathrm{HD}$ (Htt N-terminal fragment, 128Q) in vivo [42]. They also reported that RESV prevented the striatal neuronal cell death in HdhQ111 knock-in mice [42]. To test the potential neuroprotective effect of SIRT1, Jeong et al. crossed a brainspecific Sirtl knockout mice (BSKO; genotype: Sirt $1^{\text {flox/flox }}$ ) with the R6/2 HD model mice [22]. They detected the exacerbation of the neuropathological aspects of HD indicated by lower striatal (neuronal) volume [22], whereas they found the opposite in SIRT1 overexpressing knock-in mice (Sirt1KI-R6/2). These animals showed longer survival time (30\% extension) and less prominent neuropathological alterations [22]. They proposed that the neuroprotective effect of SIRT1 is exerted through the activation of the cyclic AMP response element binding transcription factor-regulated transcription coactivator 1 factor, which leads to the enhancement of the brain-derived neurotropic factor-mediated neuroprotection [22]. Jiang et al. crossed Sirtl and N171-82Q or BAC HD transgenic mice which resulted in offsprings with decelerated disease progression and reduction of brain atrophy probably via the overexpression of Sirt1 [24]. In contrast to these findings, the pharmacological inhibition of SIRT1 by selisistat exerted beneficial effects in both Drosophila and mouse models of HD and was found to be safe in human studies as well [52, 54].

SIRT2, another member of the Sirtuin family, is suspected to enhance the disease process in HD. Chopra et al. reported a beneficial effect of SIRT2 inhibition in R6/2 HD mice [7]. Previously published articles demonstrated that there is an age-dependent SIRT2 accumulation which results in microtubule deacetylation in mouse brain and spinal cord [33]. These alterations lead to the disruption of microtubuleassociated cellular transport which is an important component of the pathogenesis of HD $[10,16]$. However, it seems that the ablation of SIRT2 did not prevent the development of HD-related pathological mechanisms in R6/2 mice [5].

Similar to SIRT1, for which most of the results support a protective role in HD, SIRT3 is also proposed to have a beneficial effect regarding the pathogenesis of the disease [38], though the available data are limited. SIRT3 is involved in the regulation of fatty acid oxidation, urea- and amino acid pathways [2]. Striatal administration of a RESV dimer ( $\varepsilon$-viniferin treatment) reduced ROS level through SIRT3mediated superoxide dismutase 2 (SOD2) induction in striatal progenitor cells $(H d h(\mathrm{Q} 111))$ [14]. Some authors suggested that SIRT3 could alleviate the pathological process in HD through the deacetylation of the mitochondrial complexes (I, II, V) as well [1, 2]. Furthermore, SIRT3 is implicated in autophagy regulation via its effect on chaperones [28].

Regarding the role of another Sirtuin isoforms (SIRT4-7) in the pathogenesis of HD, experimental data are lacking.

The aim of the current study was to further elucidate the role Sirt1 and Sirt3 isoforms with presumed beneficial effects in HD, because the available data are somewhat controversial regarding Sirt1, and no deep assessment was done regarding the M1, M2 and M3 isoforms of Sirt3. For that purpose the authors applied a multi-dimensional approach to simultaneously assess the effect of the transgene, the time course of the disease and their interaction as well in addition to the screening of regional and possible gender-related differences in the N171-82Q tg mouse model of HD. 


\section{Materials and Methods}

\section{Animals}

8, 12 and 16 weeks old N171-82Q and as their control, B6C3 wt mice with identical genetic background (female and male animals distributed equally) were involved in this study ( $n=6-7$ in each group). The HD model mice originally came from Jackson Laboratories (USA). They were housed in cages under standard conditions with 12-12 h light-dark cycle. The food and water were freely available. The experiments were carried out in accordance with European Communities Council Directive (86/609/EEC) and were approved by the local animal care committee. All animals were euthanized via isoflurane overdose (Forane; Abott Laboratories Hungary Ltd., Budapest, Hungary).

\section{Sample Handling}

The brains were rapidly removed from the skull and both hemispheres were dissected into the following brain areas: striatum, cortex and cerebellum. The tissue samples were stored at $-80^{\circ} \mathrm{C}$ until the RT-PCR analysis.

\section{RT-PCR Analysis}

To perform the RT-PCR analysis, total RNA was isolated from the striatal, cortical and cerebellar samples with Trizol reagent according to manufacturer's protocol (Molecular Research Center, USA). The RNA concentration was determined with MaestroNano spectrophotometer. The RNA integrity was certified by $1 \%$ gel electrophoresis. For cDNA synthesis $1 \mu \mathrm{g}$ of total RNA, random hexamer primers and reverse transcriptase were used (Revert Aid First Strand cDNA Synthesis Kit; Thermo Scientific, USA). The synthesized cDNA was stored at $-20^{\circ} \mathrm{C}$. Real-time PCR analysis (CFX 96 Real Time System; Bio-Rad, USA) with various Sirtuin primers was performed in $20 \mu$ final volume using syber green label (PCR Biosystems, USA) [47]. Primer sequences and the exact thermal cycling conditions are described in our previous work [47]. We used the $18 \mathrm{~S}$ rRNA as endogenous control (Applied Biosystems, USA). To calculate the relative mRNA expression levels, we used the $2^{-\Delta \Delta \mathrm{Ct}}$ method [30].

\section{Statistics}

All statistical calculations were performed with the use of the freely available R software (R Development Core Team). First, the relative mRNA expression levels were calculated separately regarding each gene (i.e., Sirt1 or Sirt3), but they were normalized to the striatal level of the main subtype or isoform (i.e., Sirt1-Fl and Sirt3-M1) of 8 weeks old wt mice in each case to allow a time course analysis of the changes of expression patterns. Then we checked the distribution of data populations with the Shapiro-Wilk test and the homogeneity of variances with the Levene's test. In several cases the data diverged from Gaussian distribution and the variances were not equal. For that reason, we applied the Sheirer-Ray-Hare test to determine the differences between the investigated factors and their interaction as well. Afterwards, we carried out permutation t-tests as post hoc analysis for pairwise comparison and Type I errors from multiple comparisons were controlled with false discovery rate. As some of the possible comparisons would not have yielded meaningful information regarding the a priori decided presumptions, a maximum of 9 pairwise comparisons were implemented in case of each subtype or isoform analyzed by each brain region. The results were considered significant when the corrected $\mathrm{p}$ values were greater than 0.05 . The data were presented as median and 1 st and $3 \mathrm{rd}$ quartiles (Figs. 1, 2, 3, 4, 5).

\section{Results}

We could not detect any significant difference between male and female mice regarding the expression of any of the assessed Sirtuin isoforms either in the wt or in the $\operatorname{tg}$ groups, so they were pooled for further analyses. Furthermore, in respect of Sirt1 and Sirt3 expression, no interaction was found between the presence of the transgene and aging. Focusing on their separate effects, there was a significant elevation of Sirt1-Fl (full length) expression in all the cortical and cerebellar samples of 8,12 and 16 weeks old tg animals compared to wt mice (cortex (8 weeks: $p=0.0029 ; 12$ weeks: $p=0.0018 ; 16$ weeks: $\mathrm{p}=0.0029$ ); cerebellum ( 8 weeks: $\mathrm{p}=0.0052 ; 12$ weeks: $p=0.0054 ; 16$ weeks: $p=0.0065)$, but not in the striatum (Figs. 1 and 5). Regarding the effect of aging on Sirt1-Fl expression levels, we detected significant increase only in the cerebellum of $\operatorname{tg}$ group at 16 weeks of age ( 8 vs. 16 weeks: $p=0.0245 ; 12$ vs. 16 weeks: $p=0.0316$ ). There was no detectable change in Sirt3-M1 expression in the striatum and cortex of any age groups (8, 12, 16 weeks). In contrast, there was a clear elevation of Sirt3-M1 mRNA expression in cerebellar samples of all age groups of tg animals compared to wt mice ( 8 weeks: $\mathrm{p}=0.0024 ; 12$ weeks: $=0.0024 ; 16$ weeks: $p=0.0024 ;$ Figs. 2 and 5$)$. We could not observe any age-related effect in the Sirt3-M1 isoform in either group. Regarding Sirt3-M2 we could not detect any difference between wt and tg groups in the striatal and cortical samples, but we could identify a significant elevation in the cerebellum of tg animals in each age 
a

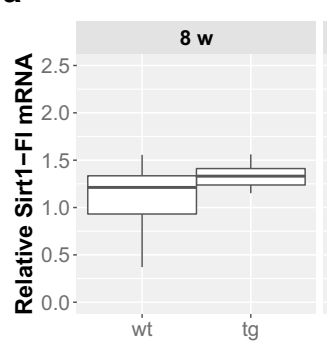

b

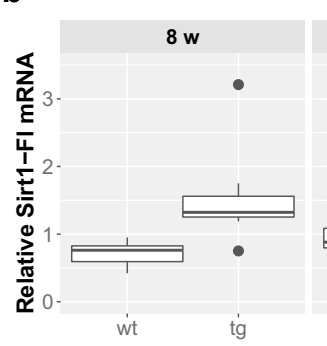

C
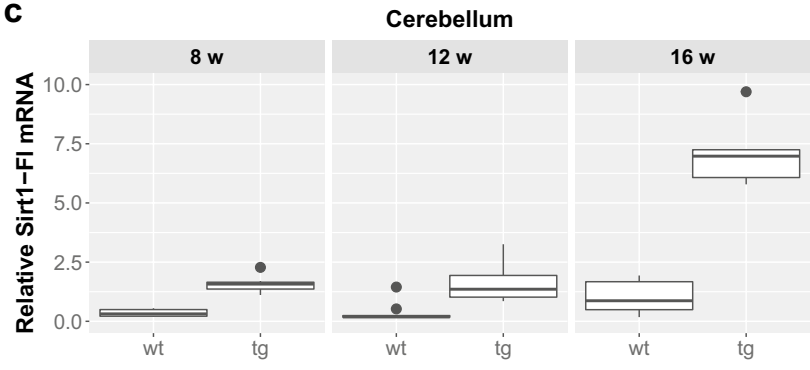

Fig. 1 Relative mRNA expression level of Sirt1-Fl in the striatum (a), cortex (b) and cerebellum (c) of N171-82Q transgenic and B6C3 wild-type mice of three age groups. The Sirt1-Fl level significantly elevated in all cortical and cerebellar samples in each age group of tg animals compared to wt mice. Aging caused a significant increase only in the cerebellum of tg group by 16 weeks of age. For clarity, the levels of significance were indicated separately in Fig. 5 in a special table format. Values are plotted as medians and interquartile ranges; $t g=$ transgenic, $w t=$ wild-type, $w=$ weeks

group compared to wt controls ( 8 weeks: $\mathrm{p}=0.0021 ; 12$ weeks: $p=0.0012 ; 16$ weeks: $p=0.0021$; Figs. 3 and 5 ). When assessing the effect of aging on Sirt3-M2 expression levels, we detected significant decrease only in the cortex of wt group at 16 weeks of age ( 8 vs. 16 weeks: $\mathrm{p}=0.038$; 12 vs. 16 weeks: $p=0.038$ ). The expression of Sirt3-M3 elevated in each striatal and cerebellar tg groups, but only in the 16 weeks old group in the tg cortex compared to $\mathrm{wt}$ mice (striatum ( 8 weeks: $\mathrm{p}=0.0097 ; 12$ weeks: $\mathrm{p}=0.0054$; 16 weeks: $\mathrm{p}=0.0097)$; cortex (16 weeks: $\mathrm{p}=0.0032)$; cerebellum ( 8 weeks: $\mathrm{p}=0.002 ; 12$ weeks: $\mathrm{p}=0.0006 ; 16$ weeks: $p=0.0016$ ) (Figs. 4 and 5). In the striatal Sirt3-M3 samples there was detectable decrease of expression by 12 weeks of age in both wt and tg animals ( 8 vs. 12 weeks (wt-wt): $\mathrm{p}=0.0243 ; 8$ vs. 12 weeks (tg-tg): $\mathrm{p}=0.036$ ). a
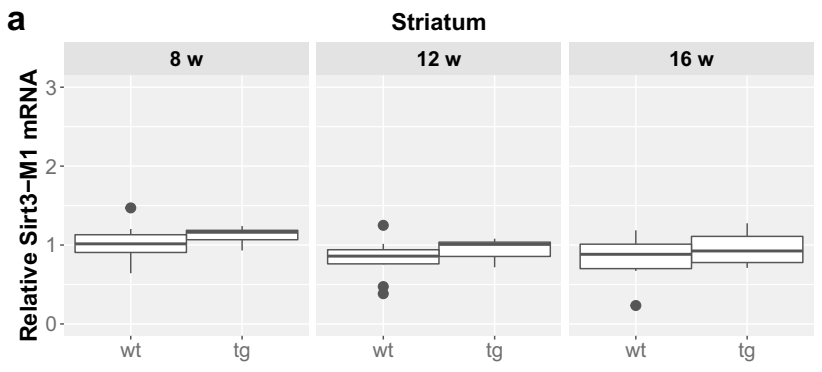

b
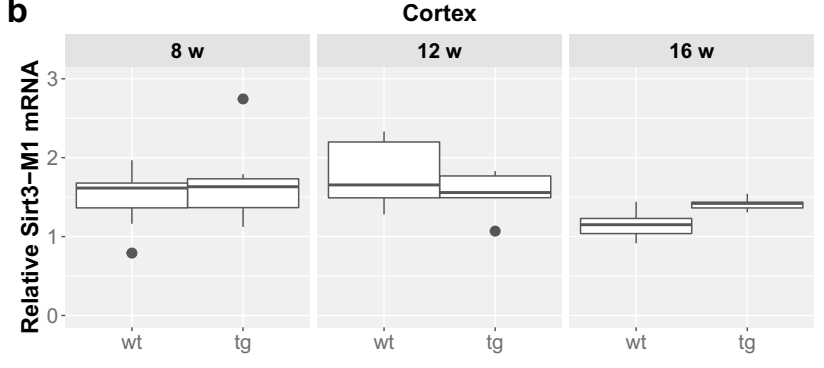

C
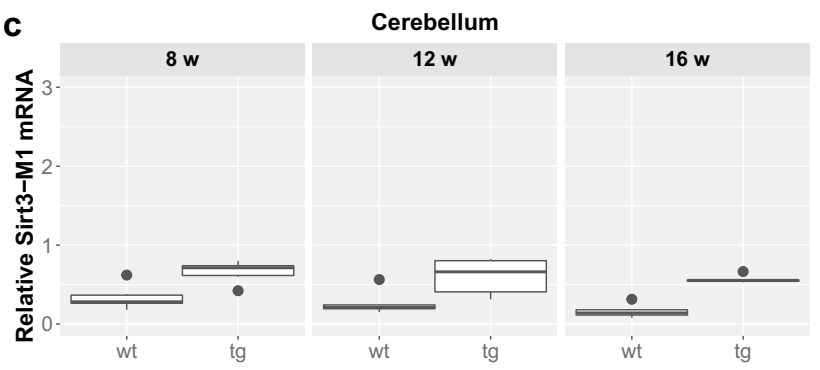

Fig. 2 Relative mRNA expression level of Sirt3-M1 in the striatum (a), cortex (b) and cerebellum (c) of N171-82Q transgenic and B6C3 wild-type mice of three age groups. The Sirt3-M1 level was significantly elevated in all cerebellar samples in each age group of tg animals compared to wt mice. For clarity, the levels of significance were indicated separately in Fig. 5 in a special table format. Values are plotted as medians and interquartile ranges; $t g=$ transgenic, $w t=$ wildtype, $w=$ weeks

\section{Discussion}

HD is a neurodegenerative disorder, which has currently no curative treatment, but encouraging clinical trials are ongoing (e.g. antisense oligonucleotide treatment) $[13,56]$. These therapeutic approaches target the pathological trinucleotide repeat expansion in the mutant hunting in mRNA, the extent of which has been demonstrated to have the strongest association with the age of disease onset and severity [12, 26, 37]. However, other important influencing factors of the disease have already been identified as well, which may serve as good candidates for the augmentation of beneficial effects obtained by the currently available therapies [32, 64].

Sirtuins are surely involved in the neurodegenerative process in HD, however, there are controversial results regarding their role [36, 38, 45, 61]. Further studies are needed to clarify these controversies and to better explore the role of 

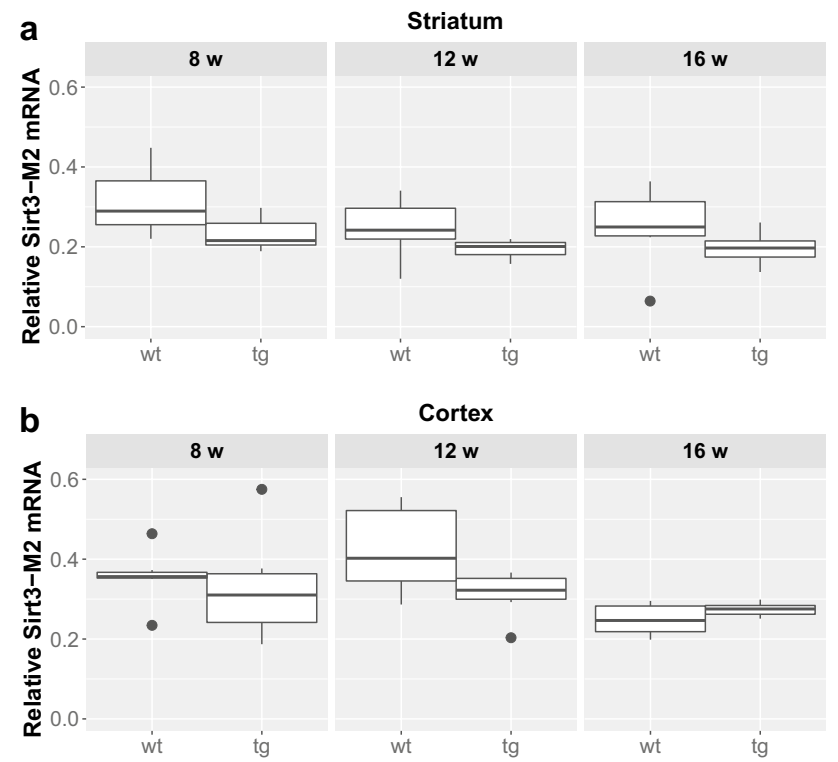

C
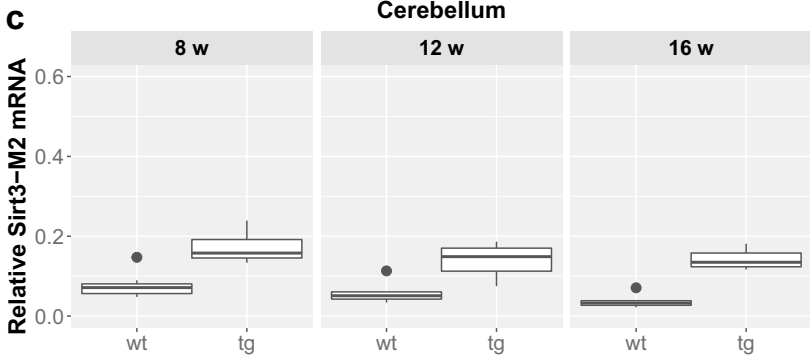

Fig. 3 Relative mRNA expression level of Sirt3-M2 in the striatum (a), cortex (b) and cerebellum (c) of N171-82Q transgenic and B6C3 wild-type mice of three age groups. The Sirt3-M2 level was significantly elevated in all cerebellar samples in each age group of tg animals compared to wt mice. Aging caused a significant decrease only in the cortex of wt group by 16 weeks of age. For clarity, the levels of significance were indicated separately in Fig. 5 in a special table format. Values are plotted as medians and interquartile ranges; $t g=$ transgenic, $w t=$ wild-type, $w=$ weeks

different Sirtuin subtypes and isoforms obtained by alternative splicing [2, 22, 24, 27, 36, 56]. Accordingly, we aimed at contributing to the clarification of the controversies regarding Sirt 1 and Sirt3 mRNA expression patterns by a better characterization of region- and aging-specific changes in the mRNA expression levels of Sirt1 and three Sirt3 isoforms using the N171-82Q tg mouse model of HD.

Tulino et al. found a significant decrease in striatal Sirt1 mRNA expression from 4 to 9 weeks in the wt group, whereas cerebellar Sirt1 mRNA expression increased significantly by 9 and 14 weeks of age in the same control group in experiments with R6/2 mouse model of HD (MRN: 204) [61]. The presence of the transgene seemingly did not affect Sirt1 mRNA expression. Another research group (Reynolds et al.) measured Sirt 1 mRNA levels in the whole-brain samples of 5, 8, 11- (MRN: 144) and 8, 12

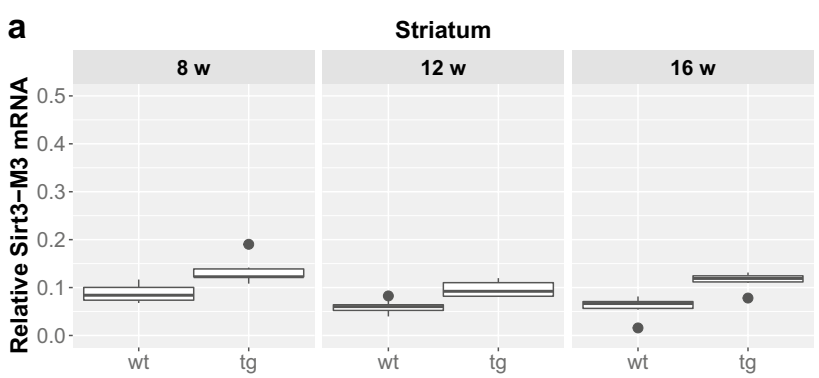

b

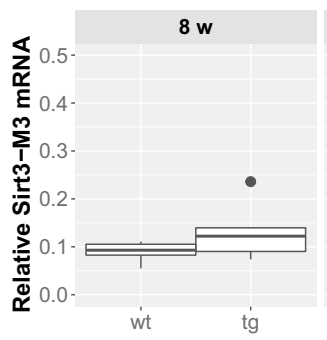

Cortex
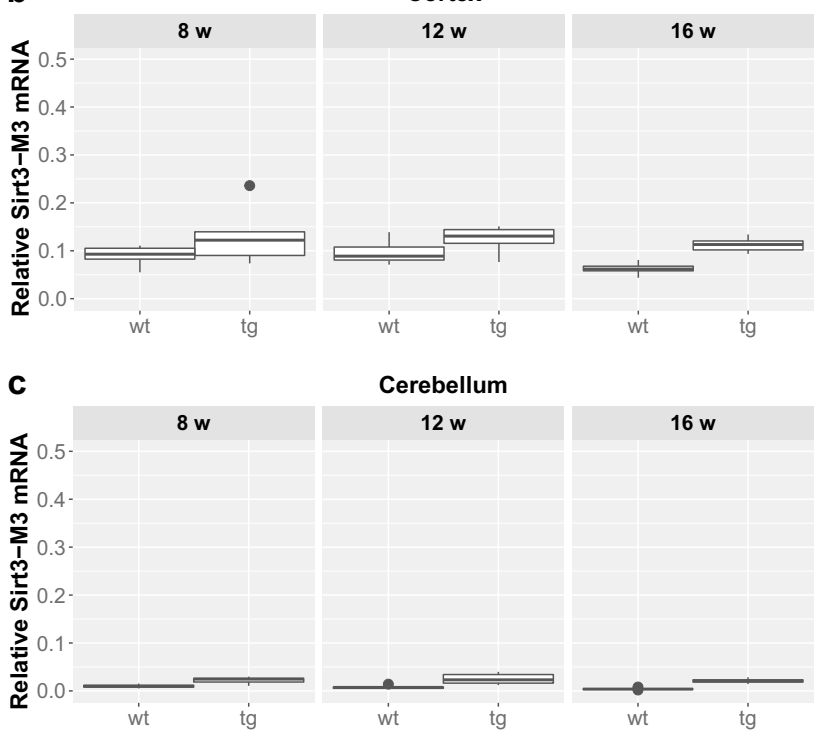

Fig. 4 Relative mRNA expression level of Sirt3-M3 in the striatum (a), cortex (b) and cerebellum (c) of N171-82Q transgenic and B6C3 wild-type mice of three age groups. The Sirt3-M3 level was significantly elevated in all striatal and cerebellar samples in each age group of tg animals compared to wt mice. The striatal expression decreased significantly by 12 weeks of age in both wt and tg animals. For clarity, the levels of significance were indicated separately in Fig. 5 in a special table format. Values are plotted as medians and interquartile ranges; $t g=$ transgenic, $w t=$ wild-type, $w=$ weeks

weeks old (MRN: 182) R6/2 mice [45]. In the 5, 8 and 11 weeks old mice (MRN: 144) there was a significant increase in mRNA levels of all tg groups. Aging did not affect the values and accordingly there was no significant interaction between age and the presence of the transgene. Regarding the only female cohort with 182 mean CAG repeat size, the significant increase could be observed only in the 8 weeks old group [45]. Due to the different CAG repeats, ages, brain regions and gender composition the comparability of these results is limited. Therefore, we find it important to get closer to the unbiased preclinical modelling of changes in Sirt 1 mRNA expression in HD. Thus, we paid a special attention to study design regarding the following points: we used the N171-82Q tg model of HD, because the phenotype of these mice mimics better the majority of human cases with considerable similarities in pathological alterations as 


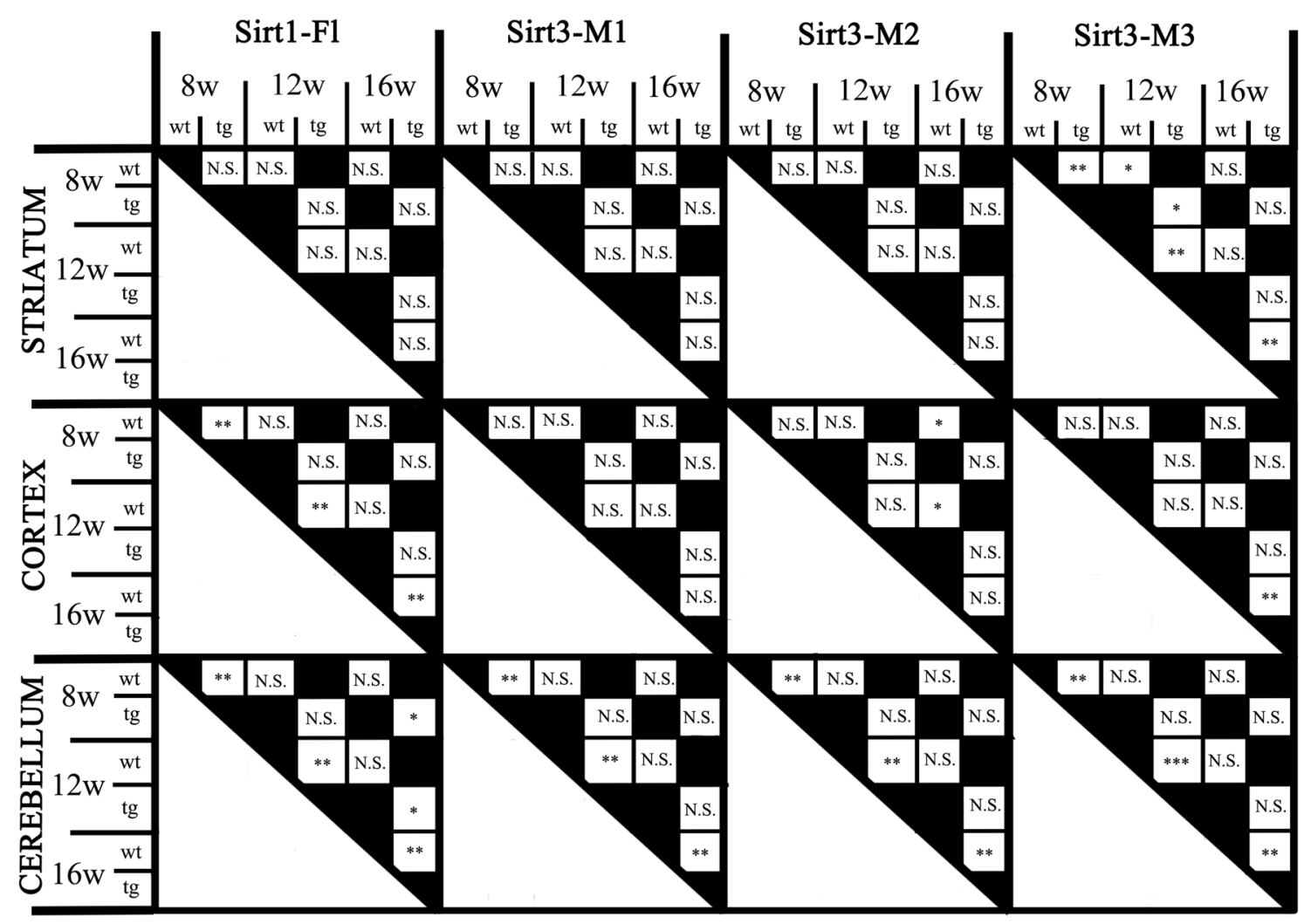

Fig. 5 The statistical delineation of the effect of the transgene and the time course of the neurodegenerative process on the expression pattern of Sirt1 and Sirt3 isoforms. In addition to the presented sta-

well than the R6/2 tg mice, the symptoms of which mainly resemble that of juvenile HD cases. Furthermore, the authors have extensive previous experiences with this model $[15,35$, $40,59,62,63,68,69]$, which enables the drawing of indirect correlations between the characteristic features of disease progression with the time course of the changes in mRNA expression patterns. Characteristically, in N171-82Q mice the symptoms begin to develop at approximately 2 months of age and disease progression results in a mean survival time of 110-130 days [68, 69]. Accordingly, the study of mice at the age of 8,12 and 16 weeks may represent early, moderate and advanced stages of the modelled disease. In addition to the assessment of the presence of transgene on Sirt mRNA expression changes in key structures (striatum, overlying cortex, cerebellum) of the regulation of motor functions, our study design involved the determination of the effect of aging and its interaction with the presence of the transgene with a view on gender-related effects as well. First of all, we found no significant differences between genders regarding either of the above-mentioned aspects, and therefore gender issues seemingly did not introduce bias into the studies of Tulino et al. and Reynolds et al. [45, 61]. Similarly to the study of Tulino et al. [61], we found no effect of the tistical differences, there were no significant interactions between the presence of the transgene and age. $* p<0.05, * * p<0.01, * * *<0.001$; $N . S .=$ not significant; $t g=$ transgenic, $w t=$ wild-type, $w=$ weeks

transgene in the striatum regarding either age groups, but a marked increase in Sirt1 expression was demonstrated in cortical and cerebellar samples of tg animals compared to wt controls in all age groups similar to that found by Reynolds et al. [45] applying whole brain samples. The magnitude of difference increased only by 16 weeks of age, and again, similarly to the latter study, no significant interaction was found between aging and the presence of the transgene. Aging-related increase in Sirt1 mRNA expression either in the striatal or cerebellar samples of wt mice, found by Tulino et al. [61], could not be confirmed by the current study.

Although data suggest that the induction of mitochondrially acting SIRT3 may be capable of exerting beneficial effects in a HD model [14], the expression pattern of Sirt3 mRNA isoforms has never been studied in any HD model. Similar to that found in case of Sirt1-Fl, a remarkable increase of cerebellar expression of all Sirt3 isoforms could be observed in tg animals compared to wt controls in the current study. The striatal expression of Sirt-M3 in all age groups and the cortical expression of Sirt3-M3 by 16 weeks of age were found to be increased. However, we have to note here that the relative expression level of Sirt3-M3 mRNA is considerably lower compared to that of the other 
two isoforms. The expression level of cortical Sirt3-M2 in wt mice and striatal Sirt3-M3 in wt and tg mice decreased by 16 and 12 weeks of age, respectively.

The pattern of expression changes in the cerebellum regarding any of the assessed Sirt subtypes and isoforms strongly resembles to that of PGC- $1 \alpha$ expression changes (either of its full length or N-terminal fragment) as we have shown in a previous study using the same animal model of HD [58]. The reason behind the same pattern may be that Sirtuins are upstream regulators of PGC- $1 \alpha$ expression $[8,25]$. Similar cerebellar predominant PGC- $1 \alpha$ activation was induced by long-term physical exercise [47] and by 1-methyl-4-phenyl-1,2,3,6-tetrahydropyridine (MPTP) treatment in its acute phase [60], but not by cooling, by shortterm exercise [47], or by 3-nitropropionic acid (3-NP), the toxin used for modeling HD-associated striatal damage [58, 65].

Although the cerebellum is known not to be the primarily affected structure in HD, there is increasing evidence of its involvement in the pathomechanism of the disorder [48]. A considerable loss of Purkinje cells was demonstrated in some HD patients with predominant motor symptoms [51], the extent of which may become more pronounced in patients with higher CAG repeat number [19]. However, there is no clear relationship between the disease stage and the degree of Purkinje and granular cell loss, and the degree of cerebellar degeneration is quite variable $[17,23,46]$. The exact background of this variability, involving the sparing of alterations as well in some human cases, is not known and needs further elucidation. Nevertheless, some studies proved cerebellar hypermetabolism in HD [44] with a presumed compensatory role for dysfunction in the fronto-striatothalamic motor circuit $[9,53]$. The significant elevations in cerebellar PGC-1 $\alpha$ [58] and Sirt mRNA expressions in the N171-82Q HD model may be considered as an important part of this compensatory cerebellar hypermetabolism. Furthermore, the increased Sirt3 mRNA expression indicates the involvement of mitochondrial activation as well. The beneficial role of the Sirt - PGC- $1 \alpha$ axis in intact cerebellar functioning may be further supported by the finding that FL-PGC- $1 \alpha$ knockout mice demonstrated reactive astrogliosis in cerebellar nuclei, whereas the striatum and cortex were almost totally spared [55]. Although data obtained from the above-mentioned toxin studies are somewhat controversial, this may be resolved by considering that in case of 3-NP the most vulnerable structures are the striatum and the hippocampus, and not the cerebellum [34], whereas MPTP is capable of inducing cerebellar degeneration as well in addition to its well-known deleterious effects on the nigrostriatal system [57]. However, the mRNA expression changes in Sirt1 and Sirt3 levels following MPTP intoxication were not significant either in its acute or chronic phase (unpublished data).
Regarding genotype-phenotype correlations, the first description of the applied N171-82Q transgenic mouse model of HD has already demonstrated that in addition to cortical, hippocampal, amygdalar and striatal involvement, an expressed deposition of intranuclear inclusions were present in cerebellar granule cells as well [49]. It was also verified by the authors, but no remarkable neuronal loss or reactive astrogliosis was found [40]. This may be explained by that the presence of abundant intranuclear inclusions does not compulsorily equals to severe neuronal damage, indeed, it was presumed to exert protective effects [3]. This may further support our hypothesis that coping mechanisms in the cerebellum, including inclusion body formation and enhanced expression pattern of the Sirt - PGC- $1 \alpha$ axis, demonstrated by the current and previous studies [59] of the authors, may be capable of exerting rather neuroprotective than damaging effects. Accordingly, the progression of the decrease of coordination, which was described as one of the key features of this model and probably attributed to cerebellar dysfunction, could be observed from 3 months of age on [20]. However, the hypokinetic feature of N171-82Q transgenic mice, rather explained by the dysfunction of the basal ganglia, involving the striatum, was still present at 8 weeks of age [69]. The evidence from unbiased anatomical work-ups of the authors also favors the above-detailed hypothesis, i.e., more than $20 \%$ of striatal neurons contained intranuclear inclusions at 16 weeks of age, whereas no remarkable neuronal loss, but prominent neuronal atrophy could be detected at this age, responsible for the decrease of striatal volume and brain weight [69]. However, besides the well demonstrated atrophy of striatum, cortex, hippocampus, hypothalamus, thalamus and amygdala, the volume of the cerebellum was still preserved even at 18 weeks of age [6].

The characteristic pattern observed in preclinical findings could not be confirmed on human samples, where Sirt1, -2, and -3 mRNA levels were assessed, demonstrating elevated cortical and striatal Sirt1 and striatal Sirt2 expression in HD patients without any change regarding Sirt3 or the cerebellum [4]. However, this gene expression study was carried out on post-mortem samples obtained from multiple centers, and the postmortem delay of sample handling and other characteristics of the specimens were not given, so their effect on individual differences in mRNA decay cannot be ruled out introducing a bias into the results. Nevertheless, further human studies are needed to be able to better determine the human relevance of the current preclinical findings.

The major limitation of the current study is the lack of experiments on protein expression changes of the corresponding Sirtuins. However, the widely applied antibodybased methods in this topic (e.g. Western blotting) are semiquantitative at best, mostly lacking appropriate sensitivity and specificity [29]. Although the application of deep proteomic analysis by mass spectrometry is appropriate, but 
quite challenging [21], especially in case of brain samples with considerably high lipid content. Accordingly, this was out of the scope of the current study. Nevertheless, future experiments are needed to confirm the results of the present work at proteome level.

In conclusion, the current study demonstrates an unequivocal elevation of Sirt1 mRNA expression level in the cortical and cerebellar samples of HD transgenic animals compared to their age-matched wild-type littermates. An increase of these differences with aging could be observed only in the cerebellum of transgenic animals, presumably related to disease progression. Regarding the expression pattern of the mitochondrially acting Sirt3 isoforms (M1, M2, M3), which have not been assessed before in HD, a similar transgenespecific increase of their cerebellar level was observed, and only the striatum showed a likewise elevation of exclusively the M3 isoform regarding the other 2 assessed brain regions. Accordingly, the observed pattern of changes with a presumed compensatory role may draw attention to that the involvement of cerebellum in HD may be more pronounced than previously thought, yielding a novel target for therapeutic approaches aiming at symptom relief in that deleterious condition.

Acknowledgements Open access funding provided by University of Szeged (SZTE). The research was supported by GINOP-2.3.2-15-201600034, EFOP-3.6.1-16-2016-00008, the Ministry of Human Capacities, Hungary [20391-3/2018/FEKUSTRAT] and the Hungarian Brain Research Program [2017-1.2.1-NKP-2017-00002 NAP VI/4] grants.

\section{Compliance with Ethical Standards}

Conflict of interest The authors declare that they have no conflict of interest.

Open Access This article is licensed under a Creative Commons Attribution 4.0 International License, which permits use, sharing, adaptation, distribution and reproduction in any medium or format, as long as you give appropriate credit to the original author(s) and the source, provide a link to the Creative Commons licence, and indicate if changes were made. The images or other third party material in this article are included in the article's Creative Commons licence, unless indicated otherwise in a credit line to the material. If material is not included in the article's Creative Commons licence and your intended use is not permitted by statutory regulation or exceeds the permitted use, you will need to obtain permission directly from the copyright holder. To view a copy of this licence, visit http://creativecommons.org/licenses/by/4.0/.

\section{References}

1. Ahn BH, Kim HS, Song S, Lee IH, Liu J, Vassilopoulos A et al (2008) A role for the mitochondrial deacetylase Sirt3 in regulating energy homeostasis. Proc Natl Acad Sci USA 105:14447-14452. https://doi.org/10.1073/pnas.0803790105

2. Ajami M, Pazoki-Toroudi H, Amani H, Nabavi SF, Braidy N, Vacca RA et al (2017) Therapeutic role of sirtuins in neurodegenerative disease and their modulation by polyphenols. Neurosci Biobehav Rev 73:39-47. https://doi.org/10.1016/j.neubi orev.2016.11.022

3. Arrasate M, Mitra S, Schweitzer ES, Segal MR, Finkbeiner S (2004) Inclusion body formation reduces levels of mutant huntingtin and the risk of neuronal death. Nature 431:805-810. https ://doi.org/10.1038/nature02998

4. Baldo B, Gabery S, Soylu-Kucharz R, Cheong RY, Henningsen JB, Englund E et al (2019) SIRT1 is increased in affected brain regions and hypothalamic metabolic pathways are altered in Huntington disease. Neuropathol Appl Neurobiol 45:361-379. https:// doi.org/10.1111/nan.12514

5. Bobrowska A, Donmez G, Weiss A, Guarente L, Bates G (2012) SIRT2 ablation has no effect on tubulin acetylation in brain, cholesterol biosynthesis or the progression of Huntington's disease phenotypes in vivo. PLoS ONE 7:e34805. https://doi.org/10.1371/ journal.pone.0034805

6. Cheng Y, Peng Q, Hou Z, Aggarwal M, Zhang J, Mori S et al (2011) Structural MRI detects progressive regional brain atrophy and neuroprotective effects in N171-82Q Huntington's disease mouse model. Neuroimage 56:1027-1034. https://doi. org/10.1016/j.neuroimage.2011.02.022

7. Chopra V, Quinti L, Kim J, Vollor L, Narayanan KL, Edgerly C et al (2012) The sirtuin 2 inhibitor AK-7 is neuroprotective in Huntington's disease mouse models. Cell Rep 2:1492-1497. https ://doi.org/10.1016/j.celrep.2012.11.001

8. Cui L, Jeong H, Borovecki F, Parkhurst CN, Tanese N, Krainc D (2006) Transcriptional repression of PGC-1alpha by mutant huntingtin leads to mitochondrial dysfunction and neurodegeneration. Cell 127:59-69. https://doi.org/10.1016/j.cell.2006.09.015

9. Deckel AW (1995) Is Huntington's disease of cerebellar/brainstem origin? Lancet 345:263-264. https://doi.org/10.1016/S0140 -6736(95)90260-0

10. Dompierre JP, Godin JD, Charrin BC, Cordelières FP, King SJ, Humbert $S$ et al (2007) Histone deacetylase 6 inhibition compensates for the transport deficit in Huntington's disease by increasing tubulin acetylation. J Neurosci 27:3571-3583. https://doi. org/10.1523/JNEUROSCI.0037-07.2007

11. Duan W, Guo Z, Jiang H, Ware M, Li XJ, Mattson MP (2003) Dietary restriction normalizes glucose metabolism and BDNF levels, slows disease progression, and increases survival in huntingtin mutant mice. Proc Natl Acad Sci USA100:2911-2916. https://doi. org/10.1073/pnas.0536856100

12. Duyao M, Ambrose C, Myers R, Novelletto A, Persichetti F, Frontali $\mathrm{M}$ et al (1993) Trinucleotide repeat length instability and age of onset in Huntington's disease. Nat Genet 4:387-392. https:// doi.org/10.1038/ng0893-387

13. Frank S (2014) Treatment of Huntington's disease. Neurotherapeutics 11:153-160. https://doi.org/10.1007/s13311-013-0244-Z

14. Fu J, Jin J, Cichewicz RH, Hageman SA, Ellis TK, Xiang L et al (2012) Trans-(-)- $\varepsilon$-Viniferin increases mitochondrial sirtuin 3 (SIRT3), activates AMP-activated protein kinase (AMPK), and protects cells in models of Huntington Disease. J Biol Chem 287:24460-24472. https://doi.org/10.1074/jbc.M112.382226

15. Gardian G, Browne SE, Choi DK, Klivenyi P, Gregorio J, Kubilus JK et al (2005) Neuroprotective effects of phenylbutyrate in the N171-82Q transgenic mouse model of Huntington's disease. J Biol Chem 280:556-563. https://doi.org/10.1074/jbc.M4102 10200

16. Gunawardena S, Her LS, Brusch RG, Laymon RA, Niesman IR, Gordesky-Gold B et al (2003) Disruption of axonal transport by loss of huntingtin or expression of pathogenic polyQ proteins in Drosophila. Neuron 40:25-40. https://doi.org/10.1016/s0896 -6273(03)00594-4 
17. Gutekunst CA, Norflus F, Hersch SM (2002) The neuropathology of Huntington's disease. In: Bates GP, Harper PS, Jones AL (eds) Huntington's disease, 4th edn. OUP, Oxford, pp 251-275

18. Hathorn T, Snyder-Keller A, Messer A (2011) Nicotinamide improves motor deficits and upregulates PGC- $1 \alpha$ and BDNF gene expression in a mouse model of Huntington's disease. Neurobiol Dis 41:43-50. https://doi.org/10.1016/j.nbd.2010.08.017

19. Hedjoudje A, Nicolas G, Goldenberg A, Vanhulle C, DumantForrest C, Deverrière G et al (2018) Morphological features in juvenile Huntington disease associated with cerebellar atrophy: magnetic resonance imaging morphometric analysis. Pediatr Radiol 48:1463-1471. https://doi.org/10.1007/s00247-018-4167-z

20. Ho DJ, Calingasan NY, Wille E, Dumont M, Beal MF (2010) Resveratrol protects against peripheral deficits in a mouse model of Huntington's disease. Exp Neurol 225:74-84. https://doi. org/10.1016/j.expneurol.2010.05.006

21. Jayasena T, Poljak A, Braidy N, Zhong L, Rowlands B, Muenchhoff $\mathrm{J}$ et al (2016) Application of targeted mass spectrometry for the quantification of sirtuins in the central nervous system. Sci Rep 6:35391. https://doi.org/10.1038/srep35391

22. Jeong H, Cohen DE, Cui L, Supinski A, Savas JN, Mazzulli JR et al (2011) Sirt1 mediates neuroprotection from mutant huntingtin by activation of the TORC1 and CREB transcriptional pathway. Nat Med 18:159-165. https://doi.org/10.1038/nm.2559

23. Jeste DV, Barban L, Parisi J (1984) Reduced Purkinje cell density in Huntington's disease. Exp Neurol 85:78-86. https://doi. org/10.1016/0014-4886(84)90162-6

24. Jiang M, Wang J, Fu J, Du L, Jeong H, West T et al (2011) Neuroprotective role of Sirt1 in mammalian models of Huntington's disease through activation of multiple Sirt1 targets. Nat Med 18:153-158. https://doi.org/10.1038/nm.2558

25. Jodeiri Farshbaf M, Ghaedi K (2017) Huntington's disease and mitochondria. Neurotox Res 32:518-529. https://doi.org/10.1007/ s12640-017-9766-1

26. Kay C, Collins JA, Miedzybrodzka Z, Madore SJ, Gordon ES, Gerry $N$ et al (2016) Huntington disease reduced penetrance alleles occur at high frequency in the general population. Neurology 87:282-288. https://doi.org/10.1212/WNL.000000000000285 8

27. La Spada AR (2012) Finding a sirtuin truth in Huntington's disease. Nat Med 18:24-26. https://doi.org/10.1038/nm.2624

28. Liang Q, Benavides GA, Vassilopoulos A, Gius D, Darley-Usmar V, Zhang J (2013) Bioenergetic and autophagic control by Sirt3 in response to nutrient deprivation in mouse embryonic fibroblasts. Biochem J 454:249-257. https://doi.org/10.1042/BJ20130414

29. Liebler DC, Zimmerman LJ (2013) Targeted quantitation of proteins by mass spectrometry. Biochemistry 52:3797-3806. https:// doi.org/10.1021/bi400110b

30. Livak KJ, Schmittgen TD (2001) Analysis of relative gene expression data using real-time quantitative PCR and the 2(-Delta Delta C(T)) method. Methods 25:402-408. https://doi.org/10.1006/ meth.2001.1262

31. Lynch CJ, Shah ZH, Allison SJ, Ahmed SU, Ford J, Warnock LJ et al (2010) SIRT1 undergoes alternative splicing in a novel auto-regulatory loop with p53. PLoS ONE 5:e13502. https://doi. org/10.1371/journal.pone.0013502

32. MacDonald ME, Vonsattel JP, Shrinidhi J, Couropmitree NN, Cupples LA, Bird ED et al (1999) Evidence for the GluR6 gene associated with younger onset age of Huntington's disease. Neurology 53:1330-1332. https://doi.org/10.1212/wnl.53.6.1330

33. Maxwell MM, Tomkinson EM, Nobles J, Wizeman JW, Amore AM, Quinti L et al (2011) The Sirtuin 2 microtubule deacetylase is an abundant neuronal protein that accumulates in the aging CNS. Hum Mol Genet 20:3986-3996. https://doi.org/10.1093/ hmg/ddr326
34. Mirandola SR, Melo DR, Saito A, Castilho RF (2010) 3-nitropropionic acid-induced mitochondrial permeability transition: comparative study of mitochondria from different tissues and brain regions. J Neurosci Res 88:630-639. https://doi.org/10.1002/ jnr.22239

35. Molnár MF, Török R, Szalárdy L, Sümegi E, Vécsei L, Klivényi P (2016) High-dose 1,25-dihydroxyvitamin D supplementation elongates the lifespan of Huntington's disease transgenic mice. Acta Neurobiol Exp (Wars) 76:176-181. https://doi.org/10.21307 lane-2017-017

36. Naia L, Rego AC (2015) Sirtuins: double players in Huntington's disease. Biochim Biophys Acta 1852:2183-2194. https://doi. org/10.1016/j.bbadis.2015.07.003

37. Nance MA (2017) Genetics of Huntington disease. Handb Clin Neurol 144:3-14. https://doi.org/10.1016/B978-0-12-80189 3-4.00001-8

38. Neo SH, Tang BL (2017) Sirtuins as modifiers of Huntington's disease (HD) pathology. Prog Mol Biol Transl 154:105-145. https ://doi.org/10.1016/bs.pmbts.2017.11.013

39. Nisoli E, Tonello C, Cardile A, Cozzi V, Bracale R, Tedesco L et al (2005) Calorie restriction promotes mitochondrial biogenesis by inducing the expression of eNOS. Science 310:314-317. https ://doi.org/10.1126/science.1117728

40. Oláh J, Klivényi P, Gardián G, Vécsei L, Orosz F, Kovacs GG et al (2008) Increased glucose metabolism and ATP level in brain tissue of Huntington's disease transgenic mice. FEBS J 275:4740 4755. https://doi.org/10.1111/j.1742-4658.2008.06612.x

41. Pallàs M, Verdaguer E, Tajes M, Gutierrez-Cuesta J, Camins A (2008) Modulation of sirtuins: new targets for antiageing. Recent Pat CNS Drug Discov 3:61-69. https://doi.org/10.2174/15748 8908783421492

42. Parker JA, Arango M, Abderrahmane S, Lambert E, Tourette C, Catoire $\mathrm{H}$ et al (2005) Resveratrol rescues mutant polyglutamine cytotoxicity in nematode and mammalian neurons. Nat Genet 37:349-350. https://doi.org/10.1051/medsci/2005215556

43. Potter MC, Yuan C, Ottenritter C, Mughal M, van Praag H (2010) Exercise is not beneficial and may accelerate symptom onset in a mouse model of Huntington's disease. PLoS Curr 2:RRN1201. https://doi.org/10.1371/currents.RRN1201

44. Rees EM, Farmer R, Cole JH, Haider S, Durr A, Landwehrmeyer B (2014) Cerebellar abnormalities in Huntington's disease: a role in motor and psychiatric impairment? Mov Disord 29:1648-1654. https://doi.org/10.1002/mds.25984

45. Reynolds RH, Petersen MH, Willert CW, Heinrich M, Nymann $\mathrm{N}$, Dall M et al (2018) Perturbations in the p53/miR-34a/SIRT1 pathway in the R6/2 Huntington's disease model. Mol Cell Neurosci 88:118-129. https://doi.org/10.1016/j.mcn.2017.12.009

46. Rodda RA (1981) Cerebellar atrophy in Huntington's disease. J Neurol Sci 50:147-57. https://doi.org/10.1016/0022510x(81)90049-6

47. Salamon A, Török R, Sümegi E, Boros F, Pesei ZG, Fort Molnár $M$ et al (2019) The effect of physical stimuli on the expression level of key elements in mitochondrial biogenesis. Neurosci Lett 698:13-18. https://doi.org/10.1016/j.neulet.2019.01.003

48. Samson M, Claassen DO (2017) Neurodegeneration and the cerebellum. Neurodegener Dis 17:155-165. https://doi. org/10.1159/000460818

49. Schilling G, Becher MW, Sharp AH, Jinnah HA, Duan K, Kotzuk JA et al (1999) Intranuclear inclusions and neuritic aggregates in transgenic mice expressing a mutant $\mathrm{N}$-terminal fragment of huntingtin. Hum Mol Genet 8:397-407. https://doi.org/10.1093/ $\mathrm{hmg} / 8.3 .397$

50. Schulte J, Littleton JT (2011) The biological function of the Huntingtin protein and its relevance to Huntington's disease pathology. Curr Trends Neurol 5:65-78 
51. Singh-Bains MK, Mehrabi NF, Sehji T, Austria MDR, Tan AYS, Tippett LJ et al (2019) Cerebellar degeneration correlates with motor symptoms in Huntington disease. Ann Neurol 85:396-405. https://doi.org/10.1002/ana.25413

52. Smith MR, Syed A, Lukacsovich T, Purcell J, Barbaro BA, Worthge SA et al (2014) A potent and selective Sirtuin 1 inhibitor alleviates pathology in multiple animal and cell models of Huntington's disease. Hum Mol Genet 23:2995-3007. https://doi. org $/ 10.1093 / \mathrm{hmg} / \mathrm{ddu} 010$

53. Squitieri F, Pustorino G, Cannella M, Toscano A, Maglione V, Morgante L (2003) Highly disabling cerebellar presentation in Huntington disease. Eur J Neurol 10:443-444. https://doi.org/10 .1046/j.1468-1331.2003.00601.x

54. Süssmuth SD, Haider S, Landwehrmeyer GB, Farmer R, Frost C, Tripepi $G$ et al (2015) An exploratory double-blind, randomized clinical trial with selisistat, a SirT1 inhibitor, in patients with Huntington's disease. Br J Clin Pharmacol 79:465-476. https://doi. org/10.1111/bcp. 12512

55. Szalardy L, Zadori D, Plangar I, Vecsei L, Weydt P, Ludolph AC (2013) Neuropathology of partial PGC- $1 \alpha$ deficiency recapitulates features of mitochondrial encephalopathies but not of neurodegenerative diseases. Neurodegener Dis 12:177-188. https://doi. org/10.1159/000346267

56. Tabrizi SJ, Leavitt BR, Landwehrmeyer GB, Wild EJ, Saft C, Barker RA (2019) Targeting Huntingtin expression in patients with Huntington's disease. N Engl J Med 380:2307-2316. https ://doi.org/10.1056/NEJMoa1900907

57. Takada M, Sugimoto T, Hattori T (1993) MPTP neurotoxicity to cerebellar Purkinje cells in mice. Neurosci Lett 150:49-52. https ://doi.org/10.1016/0304-3940(93)90105-t

58. Torok R, Konya JA, Zadori D, Veres G, Szalardy L, Vecsei L et al (2015) mRNA expression levels of PGC- $1 \alpha$ in a transgenic and a toxin model of Huntington's disease. Cell Mol Neurobiol 35:293-301. https://doi.org/10.1007/s10571-014-0124-z

59. Török R, Kónya JA, Zádori D, Veres G, Szalárdy L, Vécsei L et al (2015) mRNA expression levels of PGC-1 $\alpha$ in a transgenic and a toxin model of Huntington's disease. Cell Mol Neurobiol 35:293-301. https://doi.org/10.1007/s10571-014-0124-Z

60. Török R, Salamon A, Sümegi E, Zádori D, Veres G, Molnár MF et al (2017) Effect of MPTP on mRNA expression of PGC- $1 \alpha$ in mouse brain. Brain Res 1660:20-26. https://doi.org/10.1016/j. brainres.2017.01.032

61. Tulino R, Benjamin AC, Jolinon N, Smith DL, Chini EN, Carnemolla A et al (2016) SIRT1 activity is linked to its brain region-specific phosphorylation and is impaired in Huntington's disease mice. PLoS ONE 11:e0145425. https://doi.org/10.1371/ journal.pone.0145425

62. Vamos E, Voros K, Zadori D, Vecsei L, Klivenyi P (2009) Neuroprotective effects of probenecid in a transgenic animal model of Huntington's disease. J Neural Transm (Vienna) 116:1079-1086. https://doi.org/10.1007/s00702-009-0253-6

63. Vamos E, Voros K, Vecsei L, Klivenyi P (2010) Neuroprotective effects of L-carnitine in a transgenic animal model of Huntington's disease. Biomed Pharmacother 64:282-286. https://doi. org/10.1016/j.biopha.2009.06.020

64. van Dellen A, Hannan AJ (2004) Genetic and environmental factors in the pathogenesis of Huntington's disease. Neurogenetics 5:9-17. https://doi.org/10.1007/s10048-003-0169-5

65. Veres G, Molnár M, Zádori D, Szentirmai M, Szalárdy L, Török $\mathrm{R}$ et al (2015) Central nervous system-specific alterations in the tryptophan metabolism in the 3-nitropropionic acid model of Huntington's disease. Pharmacol Biochem Behav 132:115-124. https ://doi.org/10.1016/j.pbb.2015.03.002

66. Vonsattel JP, Myers RH, Stevens TJ, Ferrante RJ, Bird ED, Richardson EP Jr et al (1985) Neuropathological classification of Huntington's disease. J Neuropathol Exp Neurol 44:559-577. https:// doi.org/10.1097/00005072-198511000-00003

67. Yang Y, Hubbard BP, Sinclair DA, Tong Q (2010) Characterization of murine SIRT3 transcript variants and corresponding protein products. J Cell Biochem 111:1051-1058. https://doi. org $/ 10.1002 / j \mathrm{cb} .22795$

68. Zádori D, Geisz A, Vámos E, Vécsei L, Klivényi P (2009) Valproate ameliorates the survival and the motor performance in a transgenic mouse model of Huntington's disease. Pharmacol Biochem Behav 94:148-153. https://doi.org/10.1016/j. pbb.2009.08.001

69. Zádori D, Nyiri G, Szonyi A, Szatmári I, Fülöp F, Toldi J et al (2011) Neuroprotective effects of a novel kynurenic acid analogue in a transgenic mouse model of Huntington's disease. J Neural Transm (Vienna) 118:865-875. https://doi.org/10.1007/s0070 2-010-0573-6

Publisher's Note Springer Nature remains neutral with regard to jurisdictional claims in published maps and institutional affiliations. 
Research article

\title{
The effect of physical stimuli on the expression level of key elements in mitochondrial biogenesis
}

\author{
Andras Salamon ${ }^{\mathrm{a}}$, Rita Torok ${ }^{\mathrm{a}}$, Evelin Sumegi ${ }^{\mathrm{a}}$, Fanni Boros ${ }^{\mathrm{a}}$, Zsofia Gabriella Pesei ${ }^{\mathrm{a}}$, \\ Mate Fort Molnar ${ }^{\mathrm{a}}$, Gabor Veres ${ }^{\mathrm{a}}$, Denes Zadori ${ }^{\mathrm{a}}$, Laszlo Vecsei ${ }^{\mathrm{a}, \mathrm{b}}$, Peter Klivenyi ${ }^{\mathrm{a}, *}$ \\ ${ }^{a}$ Department of Neurology, University of Szeged, Semmelweis u. 6, H-6725, Szeged, Hungary \\ ${ }^{\mathrm{b}}$ MTA-SZTE Neuroscience Research Group of the Hungarian Academy of Sciences and University of Szeged, Semmelweis u. 6, H-6725, Szeged, Hungary
}

A R T I C L E I N F O

\section{Keywords:}

PGC-1 $\alpha$

Sirtuin

Training

Cold

\begin{abstract}
A B S T R A C T
Proper mitochondrial function is crucial for intact cellular homeostasis. Mitochondrial dysfunction is clearly involved in the pathogenesis of most neurodegenerative- and age-related chronic disorders. The aim of this study is to stimulate cellular production of important compounds of mitochondrial biogenesis, namely in the peroxisome proliferator-activated receptor-gamma coactivator (PGC)- and Sirtuin (SIRT)-systems.

We studied the effect of cold challenge and training on the mRNA expression levels of some compounds of these systems in different brain areas of mice. With regard to the PGC-system, the mRNA levels of the full- and Ntruncated isoforms, and those of the two promoters (brain-specific, reference) were measured. In case of Sirtuins, the mRNA levels of SIRT1 and SIRT3-M1/M2/M3 were assessed.

We found the following expression level alterations: cooling resulted in the elevation of cortical SIRT3-M1 levels and the decrease of cerebellar SIRT3-M3 levels after $200 \mathrm{~min}$. $900 \mathrm{~min}$ of cold exposure resulted in the reduction of cortical SIRT1 and striatal SIRT3-M1 levels. A prominent elevation could be observed in the levels of all PGC-1 $\alpha$ isoforms in the cerebellum after 12 days of training. The 12 days of exercise resulted in increased cerebellar SIRT3-M1 and SIRT3-M2 mRNA levels as well.

Although the efficacy of cooling core body and brain temperature is questionable, we found that training exerted a clear effect. The cause of the prominent cerebellar elevation of PGC-, and Sirtuin isoforms could be an increase in synaptic plasticity between Purkinje cells, which facilitates better motor coordination and more precise movement integration. We propose that these systems may serve as promising targets for future therapeutic studies in neurodegenerative diseases.
\end{abstract}

\section{Introduction}

A constant energy supply is crucial for proper tissue function. Mitochondria synthesize adenosine triphosphate (ATP), and play a role in adaptive thermogenesis, intracellular $\mathrm{Ca}^{2+}$ homeostasis, aging and cell death. Mitochondrial dysfunctions are implicated in the pathogeneses of neurodegenerative diseases.

Peroxisome proliferator-activated receptor-gamma (PPAR $\gamma$ ) coactivator-1 alpha (PGC-1 $\alpha$ ) is a transcriptional coactivator that regulates mitochondrial biogenesis, energy homeostasis and adaptive thermogenesis $[1,2]$. Besides the full-length protein (FL-PGC-1 $\alpha$ ), multiple PGC-1 $\alpha$ isoforms have been identified [3,4]. Alternative splicing between exons 6 and 7 of the Pgc-1 $\alpha$ gene produces the N-truncated, shorter PGC-1 $\alpha$ (NT-PGC-1 $\alpha$ ) isoform [5]. Besides the classical proximal promoter, novel tissue-specific PGC- $1 \alpha$ isoforms have recently been described, including muscle-, liver- and central nervous systemspecific (CNS-PGC-1 $\alpha$ ) isoforms $[3,4,6,7]$.

Sir2-like proteins (Sirtuins) are mainly $\mathrm{NAD}^{+}$-dependent deacetylases which play a prominent role in mitochondrial biogenesis $[8,9]$. Seven mammalian Sirtuin subtypes (SIRT1-7) that are present in different subcellular locations were identified [10]. Alternative promoter usage and splicing variability results in a wide range of Sirtuin isoforms. From the perspective of our research, the four most important subtypes are SIRT1 and SIRT3-M1, -M2 and -M3 [11-14].

It is well known that tissue-specific PGC-1 $\alpha$ and Sirtuin alterations develop by challenging the energy homeostasis, e.g., with cold exposure and physical exercise [1,6,15-27]. Previous studies described that full length PGC-1 $\alpha$ mRNA expression was elevated in mouse brown adipose tissue (BAT) and skeletal muscle following cold exposure [1]. It is also known that cold exposure shifts the transcription from PP to an

\footnotetext{
* Correspondent author.

E-mail address: klivenyi.peter@med.u-szeged.hu (P. Klivenyi).
} 
alternative promoter in BAT [6]. Recent studies demonstrated that the mRNA levels of SIRT2 and SIRT3 in mice BAT were increased by cold exposure $\left(3,6,12 \mathrm{~h}\right.$ periods at $\left.5^{\circ} \mathrm{C}\right)$ and decreased if the environmental temperature was higher than thermoneutral $\left(16 \mathrm{~h}\right.$ period at $\left.27.5^{\circ} \mathrm{C}\right)$ [22]. SIRT6 mRNA and protein levels were also elevated in the brown and inguinal white adipose tissue of 8 -week-old mice following $4{ }^{\circ} \mathrm{C}$ overnight cold exposure [25]. While there is increasing data about changes in these 2 systems in BAT following cooling, there is little data related to alterations in the brain. A study demonstrated that the brain mRNA level of PGC-1 $\alpha$ did not change after $3 \mathrm{~h}$ or $12 \mathrm{~h}$ at $4{ }^{\circ} \mathrm{C}$ [26].

Increased PGC-1 $\alpha$ expression was observed in skeletal muscles after physical exercise [24,27]. An isoform-specific expression pattern during exercise of different intensity was demonstrated, which is caused by a promoter shift $[22,24]$. It has been proven that PGC-1 mRNA levels increased in rats already on the first day of a 1-week training program where the rats trained on alternate days [20]. However, following a similar increase on the first day, an opposite, decreasing trend was observed with 4 days of consecutive training [20]. Studies reported that long-term intensive exercise training induced FL-PGC-1 $\alpha$ expression and mitochondrial biogenesis in the whole brain, particularly in some brain areas of mice [23]. The 17-day-long training did not change PGC$1 \alpha$ protein levels in the examined brain regions (cortex, striatum), either in young or old mice [18].

Although exercise failed to extend life span in animal experiments, it seemingly had an influence in the Sirtuin-system [15-23]. Several studies have been carried out to investigate the correlation between training intensity, the age of animals and Sirtuin expression in the skeletal muscle, liver and heart $[15,21]$. The results of these studies can be summarized as an increase in SIRT1 and SIRT6 levels after exercise with different protocols [39]. Steiner and Bayod found an elevation of SIRT1 protein levels in specific brain regions of mice after exercise $[15,23]$, whereas Lezi et al. found no difference in the expression level of SIRT1 in brains of mice following exercise [40]. With regards to another subtype of the Sirtuin-system, SIRT3, its level was found to be elevated in the brain of exercised mice [16].

It has already been demonstrated that there is a direct relationship between the Sirtuin- and PGC-systems [28,29]. These systems are implicated in neurodegenerative diseases, such as ALS, Huntington's, Parkinson's and Alzheimer's disease [30].

Although the expression of PGC-1 $\alpha$ and Sirtuins have been already investigated in rodent brain with the above-mentioned environmental stimuli, the results are controversial [1,6,15-27]. From the perspective of the PGC-system, only the FL-PGC-1 $\alpha$ and NT-PGC-1 $\alpha$ were previously examined, whereas it is well established that in the brain, shortterm cold exposure did not alter the level of the examined isoforms. Training elevated the levels of PGC- $1 \alpha$ isoforms in intensity-, age- and duration-specific manners. However, there is no available data about the recently identified novel isoforms of PGC- and Sirtuin-systems in different brain regions. Accordingly, the aim of the current study was to assess the effect of cold challenge and training on the expression levels of FL-PGC-1 $\alpha$, NT-PGC-1 $\alpha$, CNS-PGC-1 $\alpha$, PP-PGC-1 $\alpha$, SIRT1, SIRT3-M1, $-\mathrm{M} 2$ and $-\mathrm{M} 3$ in the striatum, cortex and cerebellum of wild-type C57Bl/ $6 \mathrm{~J}$ mice.

\section{Experimental procedure}

\subsection{Animals}

20 -week-old female $\mathrm{C} 57 \mathrm{Bl} / 6 \mathrm{~J}$ mice were involved in this study. The rationale for the use of female mice was that the PGC-system has a gender-specific expression pattern [31,32]. Weydt et al. detected an SNP (rs3736265, PPARGC1A) in patients with Huntington's disease which caused earlier disease onset only in men [32]. They also showed that there is an earlier disease onset and age of death in SOD1 transgenic ALS model of FL-PGC-1 $\alpha$ deficient male mice [31]. Accordingly, these data indicate that the PGC-system mediated protective effects may be more active in females. With regards to the Sirtuin-system, it can be said that the overexpression of SIRT6 increases lifespan in transgenic male mice, but not in females. As a conclusion, the Sirtuinsystem seems to be more active in female mice as well [33]. The animals were originally obtained from Jackson Laboratory (Bar Harbor, ME, USA). The mice were housed in cages under standard conditions with $12-12 \mathrm{~h}$ light-dark cycle and free access to food and water. The experiments were carried out in accordance with the European Communities Council Directive (86/609/EEC) and were approved by the local animal care committee. All animals were euthanized via isoflurane overdose (Forane; Abott Laboratories Hungary Ltd., Budapest, Hungary).

\subsection{Treatments and sample handling}

\subsubsection{Cold exposure}

Animals were randomly divided into four groups $(n=7-8$ in each group). The first group was kept at $4{ }^{\circ} \mathrm{C}$ for $40 \mathrm{~min} /$ day, for 5 days (200 min), the second one was kept $4{ }^{\circ} \mathrm{C}$ for $180 \mathrm{~min} /$ day for 5 days ( $900 \mathrm{~min}$ ). After the cold exposure, mice were placed back under standard conditions $\left(22-24^{\circ} \mathrm{C}\right)$. The third and fourth groups were control groups and were housed at $22-24^{\circ} \mathrm{C}$ in the same room. Ninety minutes after the last cold exposure the animals were deeply anesthetized with isoflurane and their brains were dissected immediately.

\subsubsection{Exercise training}

Exercise training was examined using a rotarod. The mice were randomly allocated into four groups ( $n=5-8$ in each group). The first and second groups were the training groups. The mice were placed on the rotarod for a 2 -session period ( 9.00 a.m., 4.00 p.m.) for 5 days (first group) or 12 days (second group). The speed profile was standard 5 RPM for $30 \mathrm{~min}$. Prior to training, the mice were transported to the testing room for an acclimatization period of at least $30 \mathrm{~min}$. The third and fourth groups were control groups. Ninety minutes after the last measurement, the animals were anesthetized, and the brains were dissected immediately.

\subsection{RT-PCR analysis}

Total RNA was isolated from striatum, cortex and cerebellum with Trizol according to the manufacturer's protocol. RNA concentrations were measured with a MaestroNano spectrophotometer, and the integrity of RNA was confirmed by gel electrophoresis using $1 \%$ agarose gel. cDNA was generated from $1 \mu \mathrm{g}$ of total RNA with random hexamer primers and reverse transcriptase according to the Revert Aid First Strand cDNA Synthesis Kit protocol (Thermo Scientific, USA). cDNA was kept at $-20^{\circ} \mathrm{C}$ until further use. Real-time PCR was performed with a CFX 96 Real TimeSystem (Bio-Rad, USA) to detect changes in mRNA expression, using various primer pairs at a final volume of $20 \mu \mathrm{l}$. We used previously described PGC- $1 \alpha$ and Sirtuin primers $[6,12,14]$. (See the exact thermal cycling conditions in Supplementary File 1.). Target gene expression was normalized to the endogenous control gene 18S rRNA (Applied Biosystems, USA). Relative expression was calculated by the $2^{-\Delta \Delta \mathrm{Ct}}$ method [34].

\subsection{Statistics}

All statistical analyses were performed with the use of the R software (R Development Core Team). The Levene test was performed for the analysis of homogeneity of variances. To assess the differences between PGC-1 $\alpha$ - and Sirtuin gene expression levels in all brain areas relative to their respective control groups, approximative (10 000 random permutation) two sample Fisher-Pitman permutation test was applied. We calculated the gene expression level of PGC-transcripts in all brain areas relative to FL-PGC- $1 \alpha$ and CNS-PGC- $1 \alpha$ control striatum groups. For Sirtuins we compared SIRT1 expression levels to SIRT1-FL, 
and all SIRT3 isoforms to SIRT3-M1 control striatum groups. The differences were considered significant when the $\mathrm{p}$ values were less than 0.05 .

\section{Results}

\subsection{PGC-1 $\alpha$ transcript levels}

\subsubsection{Cold exposure}

There were no detectable changes in the levels of PGC- $1 \alpha$ transcripts in the different brain areas after the total $200 \mathrm{~min}$ or $900 \mathrm{~min}$ cold exposure. The expression level of all the investigated transcripts was detected at room temperature and this expression was not altered by cold exposure (Supplementary Figs. 1 and 2).

\subsubsection{Exercise training}

The levels of PGC-1 $\alpha$ transcripts did not show any change in the investigated brain areas after the 5-day-long rotarod training (Supplementary Fig. 3). However, the 12 day exercise training resulted in significant increases in FL-PGC-1 $\alpha$, NT-PGC- $1 \alpha$, CNS-PGC-1 $\alpha$ and Ref-PGC-1 $\alpha$ mRNA expression in the cerebellum (FL-PGC-1 $\alpha$ : ctrl: $1.32 \pm 0.20 ; \quad$ EX: $1.59 \pm 0.19 ; \quad \mathrm{p}=0.024 ; \quad$ NT-PGC-1 $\alpha: \quad$ ctrl: $0.29 \pm 0.04 ; \quad$ EX: $0.38 \pm 0.04 ; \quad p=0.0002 ; \quad$ CNS-PGC- $1 \alpha: \quad$ ctrl: $1.35 \pm 0.23 ; \quad$ EX: $1.80 \pm 0.32 ; \quad \mathrm{p}=0.003, \quad$ Ref-PGC- $1 \alpha: \quad$ ctrl: $0.21 \pm 0.03$; EX: $0.30 \pm 0.02 ; \mathrm{p}=0.0003$; Fig. $1 \mathrm{C}$ ). With regards to the striatum and the cortex, no other significant differences were detected (Fig. $1 \mathrm{~A}, \mathrm{~B}$ ). To verify that the CNS specific promoter is only poorly expressed in peripheral tissues, the quadriceps muscle was used,

\section{A. Striatum}

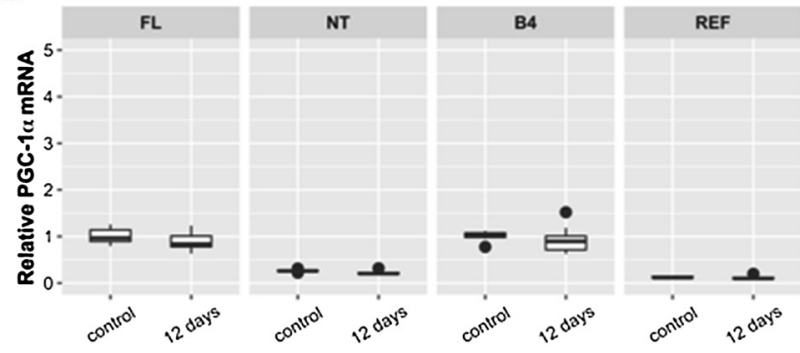

\section{B. Cortex}

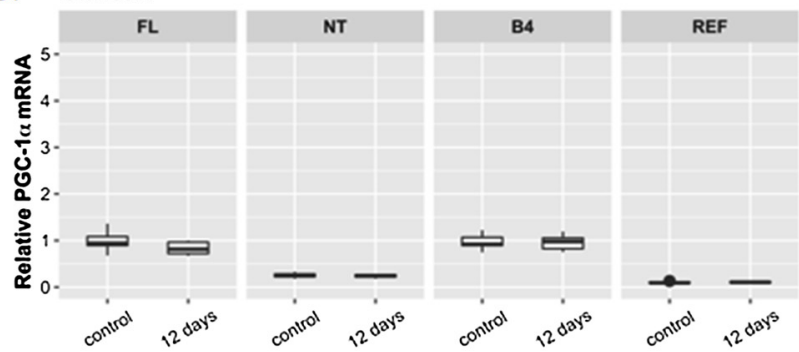

\section{Cerebellum}

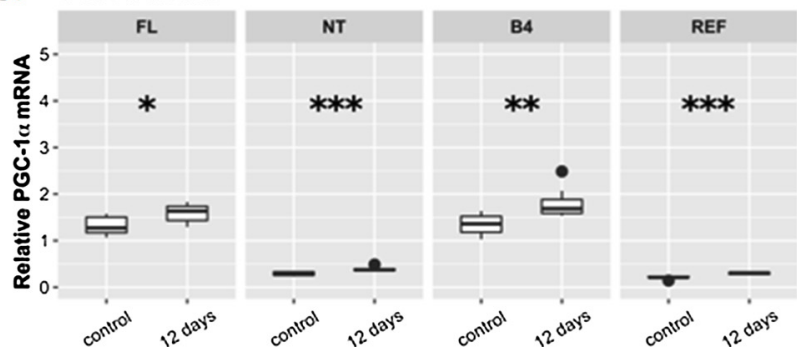

demonstrating hardly detectable expression levels. However, the expression level of FL-PGC-1 $\alpha$ (ctrl: $1.01 \pm 0.19$; EX: $3.19 \pm 1.25$; $\mathrm{p}=0.003), \quad$ NT-PGC- $1 \alpha \quad$ (ctrl: $0.10 \pm 0.02 ; \quad$ EX: $0.50 \pm 0.19$; $\mathrm{p}=0.001$ ) and Ref-PGC-1 $\alpha$ (ctrl: $1.00 \pm 0.11$; EX: $1.69 \pm 0.52$; $\mathrm{p}=0.016)$ mRNA was significantly elevated in the quadriceps muscle after 5 days of training.

\subsection{Sirtuin transcript levels}

\subsubsection{Cold exposure}

After $200 \mathrm{~min}$ of cold exposure there were no detectable changes in the levels of SIRT1 and SIRT3-M2 transcripts in any brain regions (Fig. 2), but SIRT3-M1 levels elevated in the cortex (ctrl: $1.26 \pm 0.49$; EX: $1.97 \pm 0.60 ; p=0.036$; Fig. 2 B), whereas SIRT3-M3 levels decreased in the cerebellum (ctrl: $0.16 \pm 0.05$; EX: $0.10 \pm 0.03$; $\mathrm{p}=0.027$; Fig. $2 \mathrm{C}$ ). $900 \mathrm{~min}$ of cooling resulted in the relative decrease of cortical SIRT1 (ctrl: $1.14 \pm 0.31$; EX: $0.66 \pm 0.24$; $\mathrm{p}=0.008$; Fig. $2 \mathrm{E}$ ) and striatal SIRT3-M1 (ctrl: $1.04 \pm 0.30$; EX: $0.72 \pm 0.21 ; \mathrm{p}=0.029$; Fig. 2 D) expression levels.

\subsubsection{Exercise training}

After 5 days of rotarod training, cortical SIRT1 levels were found to be elevated (ctrl: $0.78 \pm 0.10$; EX: $0.97 \pm 0.16$; $p=0.042$ ), but the other isoforms did not change (Supplementary Fig. 4).

However, 12 days of exercise training resulted in the increase of both SIRT3-M1 and SIRT3-M2 mRNA expression in the cerebellum (SIRT3-M1: ctrl: $0.79 \pm 0.18$; EX: $1.28 \pm 0.30 ; p=0.002$; SIRT3-M2: ctrl: $0.33 \pm 0.09$; EX: $0.50 \pm 0.10 ; p=0.007$; Fig. 1 F). We did not

\section{Striatum}

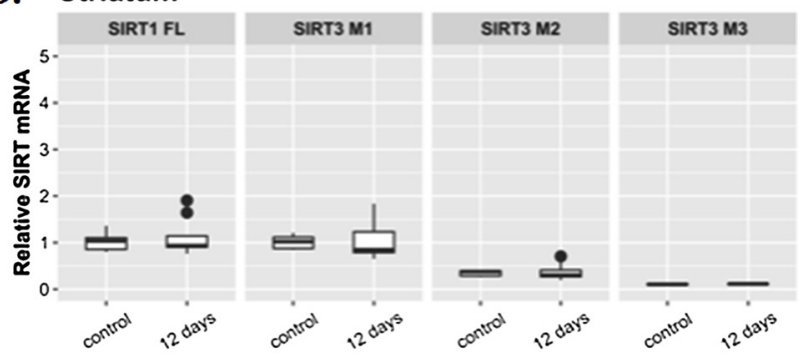

\section{E. Cortex}

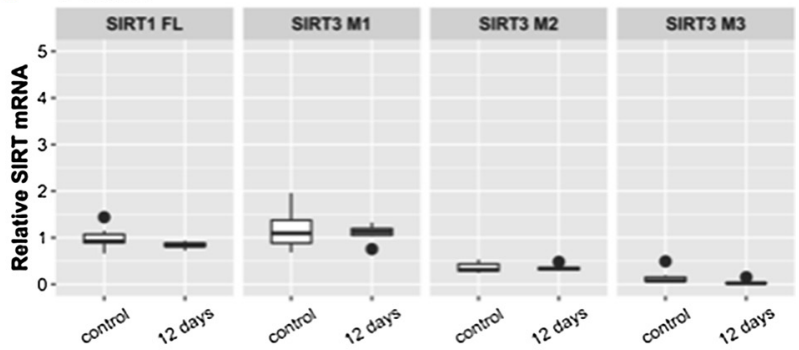

\section{F. Cerebellum}

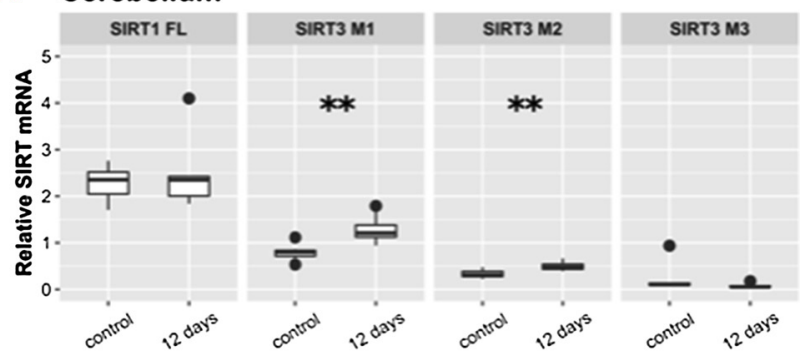

Fig. 1. Striatal (A, D), cortical (B, E) and cerebellar (C, F) relative mRNA expression levels of PGC1-1 $\alpha$ (A, B, C) and Sirtuin (D, E, F) isoforms in mice after 12 days of rotarod training (5 RPM). The FL-PGC-1 $\alpha$, NT-PGC-1 $\alpha$, CNS-PGC-1a (B4), Reference promoter (REF), SIRT3-M1 and -M2 levels significantly increased in the cerebellum of exercised mice. Values are plotted as medians and interquartile range; ${ }^{*} \mathrm{p}<0.05$, ${ }^{* *} \mathrm{p}<0.01$, ${ }^{* * *} \mathrm{p}<0.005 ; 12 D-12$ days rotarod training; str striatum; $c t x$ - cortex; $c r b$ - cerebellum. 
A. Striatum

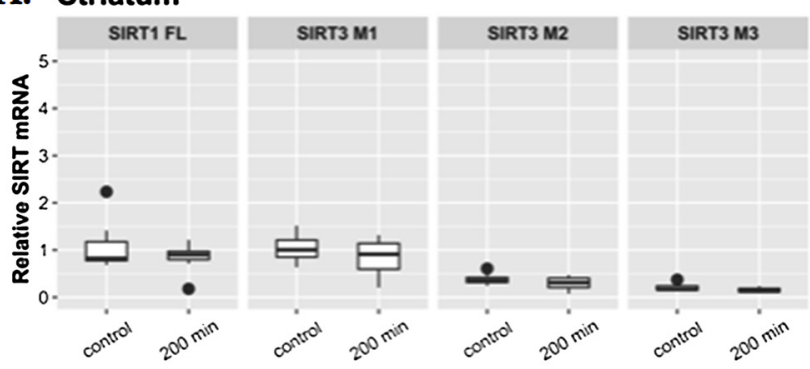

B. Cortex

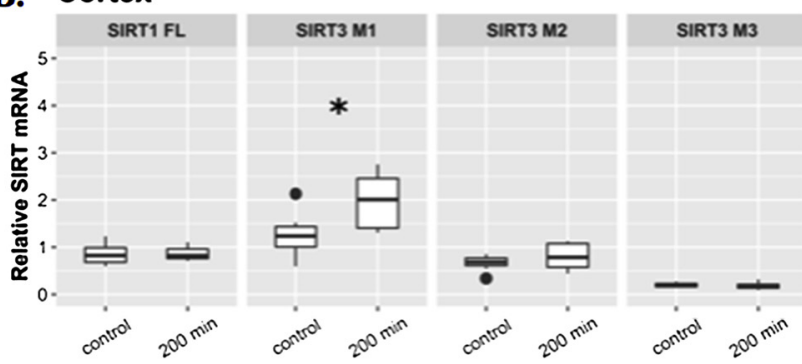

C. Cerebellum

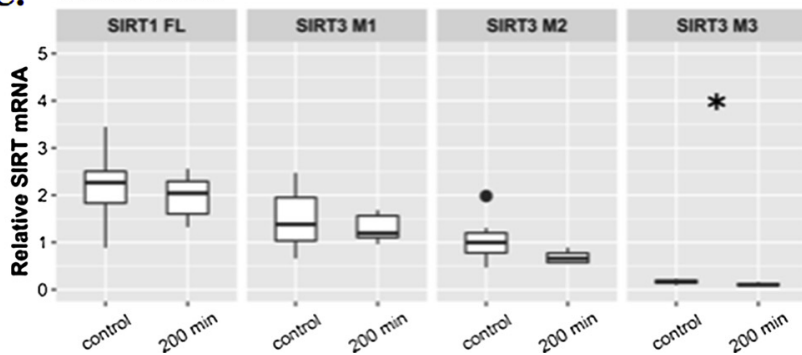

\section{Striatum}

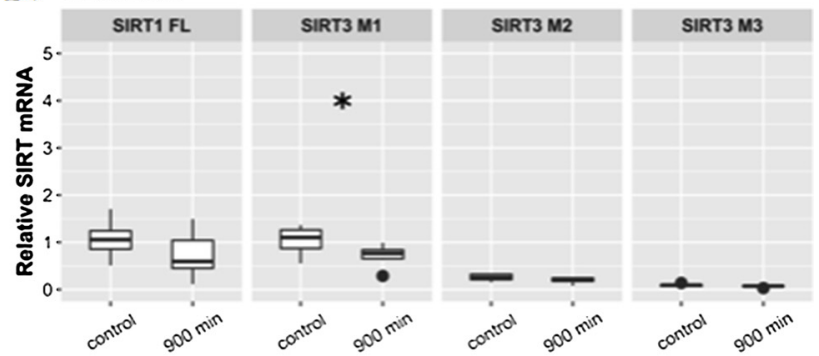

\section{E. Cortex}

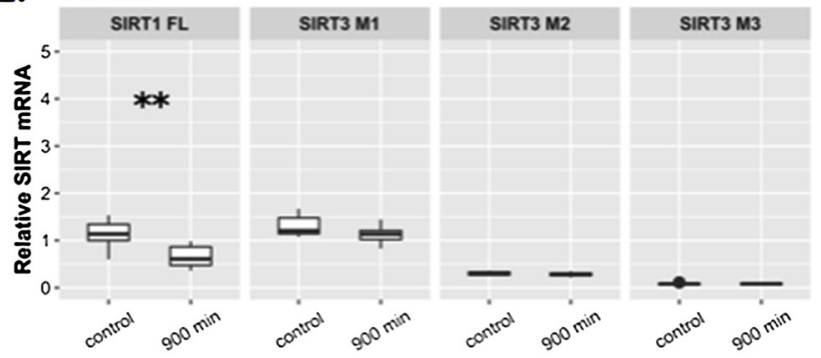

\section{Cerebellum}

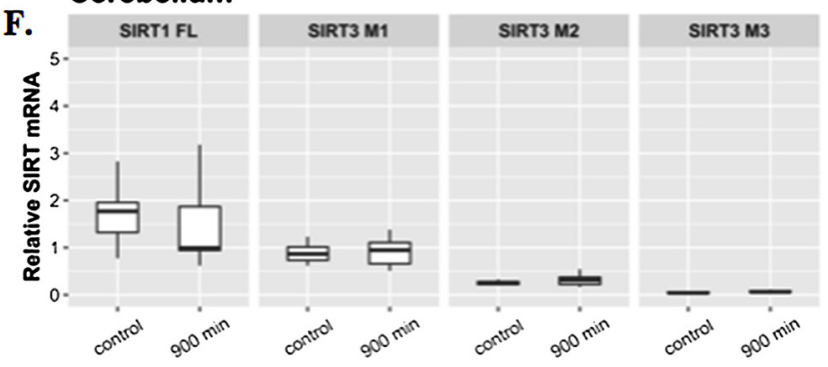

Fig. 2. Striatal, (A, D), cortical (B, E) and cerebellar (C, F) relative mRNA expression levels of Sirtuin isoforms in mice after 200 (A-C) and 900 (D-F) minutes of cold exposure $\left(4^{\circ} \mathrm{C}\right)$. After $200 \mathrm{~min}$ the SIRT3-M1 isoform was significantly upregulated in mice cortex and cerebellar SIRT3-M3 was also significantly decreased. After 900 min the SIRT1-FL isoform was significantly downregulated in mice cortex and striatal SIRT3-M1 was also significantly decreased. Values are plotted as medians and interquartile range; ${ }^{*} \mathrm{p}<0.05,{ }^{* *} \mathrm{p}<0.01,{ }^{* * *} \mathrm{p}<0.005 ; 200-200 \mathrm{~min}$ cold exposure; str - striatum; ctx - cortex; crb - cerebellum.

find differences in SIRT1 and SIRT3-M3 levels in any other brain areas (Fig. 1 D, E).

\section{Discussion}

Maintenance of energy homeostasis is crucial for survival. PGC-1 $\alpha$ and Sirtuins modulate mitochondrial biogenesis and other cellular mechanisms in the brain in response to physical exercise and cold exposure. In this study we investigated the isoform- and brain area-specific expression pattern of PGC-1 $\alpha$ and Sirtuin following environmental stimuli.

The alteration of PGC- $1 \alpha$ has already been examined in the brain, and previous findings suggested that very short-term cold exposure did not influence PGC-1 $\alpha$ expression in the brain [26]. Accordingly, we also could not demonstrate any changes in the FL-PGC-1 $\alpha$, NT-PGC-1 $\alpha$, CNS-PGC-1 $\alpha$ or Ref-PGC- $1 \alpha$ levels in any brain area after cold exposure between the control and short- or long-duration cold-exposed animals.

However, there is currently no data about the effect of cooling on the levels of Sirtuin isoforms in the brain. We found that short exposure ( $200 \mathrm{~min}$ ) elevated the level of the SIRT3-M1 isoform in the cortex, and decreased the SIRT3-M3 level in the cerebellum. The long exposure (900 min) revealed a decline in cortical SIRT1, and striatal SIRT3-M1 levels. As an explanation, we suppose that this cold-challenge regime is not effective in decreasing the core body temperature sufficiently and the early compensatory mechanisms in BAT and skeletal muscle protect the brain against cold exposure.

Previous studies reported that physical activity reduces the risk of dementia and Alzheimer's disease [35]. The possible effects of inactivity are impaired learning and memory functions, dementia and neurodegeneration [36]. It is well-known that exercise increases mitochondrial biogenesis via the up-regulation of PGC-1 $\alpha$ and Sirtuin pathways in various tissues.

Previous studies demonstrated that metabolic stress occurring in the brain during exercise is similar to that known to stimulate mitochondrial biogenesis in muscle. Therefore, the effects of exercise training on PGC- $1 \alpha$ have been examined in the brain as well, but the results are inconsistent. Lezi et al. could not detect any alteration of PGC-1 $\alpha$ mRNA levels in young or old mice following exercise training. Contrarily, another group reported a considerable elevation of PGC-1 $\alpha$ mRNA in different brain areas, but the training protocols were different [23].

From the perspective of Sirtuins it seems that in the brain there could be an elevation in the expression levels of SIRT1 and SIRT6, but the available data are controversial $[15,16,23,40]$. In this study, we investigated FL-PGC- $1 \alpha$, NT-PGC- $1 \alpha$, CNS-PGC- $1 \alpha$, and Ref-PGC- $1 \alpha$ mRNA levels in two different training protocols. The 5-day-long training period did not cause alterations in PGC-1 $\alpha$ transcripts in any brain regions. Contrarily, the 12-day-long training period induced changes in all isoforms of the PGC-system in the cerebellum. In the Sirtuin-system, the 5-day-long training also did not cause mRNA level alterations, but the long-term exercise resulted cerebellar elevation of SIRT3-M1 and SIRT3-M2 mRNA levels. These results suggest that very short-term exercise was unable to induce the PGC-1 $\alpha$ and SIRT systems. Contrarily, the 12-day-long training period induced changes in the cerebellum, which seems to be consistent with our previously findings [37]. We hypothesize that the reason behind the prominent cerebellar 
elevation of PGC-, and Sirtuin isoforms could be the increase in synaptic plasticity between Purkinje cells, which facilitates better motor coordination and more precise movement integration. Previous studies demonstrated that in the cerebellum of $P g c$ knockout mice there is a decrease in cell number and firing rate between Purkinje cells [18,38].

In conclusion we suggest that all PGC isoforms and SIRT3-M1,-M2 (i.e., the mitochondrial Sirtuins, except SIRT3-M3, which seems does not play important role in the cerebellum) take part in mitochondrial energy production, enhancing synaptic functioning. However, additional studies are needed to better understand the interaction between mitochondria and each PGC- and Sirtuin isoform in the cerebellum.

\section{Funding sources}

The current work was supported by Hungarian Brain Research Program - Grant No. 2017-1.2.1-NKP-2017-00002_VI/4 and GINOP2.3.2-15-2016-00034. Dénes Zádori was supported by the János Bolyai Research Scholarship of the Hungarian Academy of Sciences.

\section{Conflict of interest}

The authors declare that there is no conflict of interest.

\section{Appendix A. Supplementary data}

Supplementary material related to this article can be found, in the online version, at doi:https://doi.org/10.1016/j.neulet.2019.01.003.

\section{References}

[1] P. Puigserver, Z. Wu, C.W. Park, R. Graves, M. Wright, B.M. Spiegelman, A coldinducible coactivator of nuclear receptors linked to adaptive thermogenesis, Cell 92 (1998) 829-839, https://doi.org/10.1016/S0092-8674(00)81410-5.

[2] P. Puigserver, B.M. Spiegelman, Peroxisome proliferator-activated receptor- $\gamma$ coactivator $1 \alpha(\mathrm{PGC}-1 \alpha)$ : transcriptional coactivatior and metabolic regulator, Endocr. Rev. 24 (2003) 78-90, https://doi.org/10.1210/er.2002-0012.

[3] J.L. Ruas, J.P. White, R.R. Rao, S. Kleiner, K.T. Brannan, B.C. Harrison, N.P. Greene, J. Wu, J.L. Estall, B.A. Irving, L.R. Lanza, K.A. Rasbach, M. Okutsu, K.S. Nair, Z. Yan, L.A. Leinwand, B.M. Spiegelman, A PGC-1 $\alpha$ isoform induced by resistance training regulates skeletal muscle hypertrophy, Cell 151 (2012) 1319-1331, https://doi.org/10.1016/j.cell.2012.10.050.

[4] V. Martínez-Redondo, A.T. Pettersson, J.L. Ruas, The hitchhiker's guide to PGC-1 $\alpha$ isoform structure and biological functions, Diabetologia 58 (2015) 1969-1977, https://doi.org/10.1007/s00125-015-3671-z.

[5] Y. Zhang, P. Huypens, A.W. Adamson, J.S. Chang, T.M. Henagan, A. Boudreau, N.R. Lenard, D. Burk, J. Klein, N. Perwitz, J. Shin, M. Fasshauer, A. Kralli, T.W. Gettys, Alternative mRNA splicing produces a novel biologically active short isoform of PGC-1alpha, J. Biol. Chem. 284 (2009) 32813-32826, https://doi.org/ 10.1074/jbc.M109.037556.

[6] J.S. Chang, V. Fernand, Y. Zhang, J. Shin, H.J. Jun, Y. Joshi, T.W. Gettys, NT-PGC1alpha protein is sufficient to link beta3-adrenergic receptor activation to transcriptional and physiological components of adaptive thermogenesis, J. Biol. Chem. 287 (2012) 9100-9111, https://doi.org/10.1074/jbc.M111.320200.

[7] S.M. Soyal, T.K. Felder, S. Auer, P. Hahne, H. Oberkofler, A. Witting, M. Paulmichl, G.B. Landwehrmeyer, P. Weydt, W. Patsch, European Huntington Disease Network, A greatly extended PPARGC1A genomic locus encodes several new brain-specific isoforms and influences Huntington disease age of onset, Hum. Mol. Genet. 21 (2012) 3461-3473, https://doi.org/10.1093/hmg/dds177.

[8] Y. Cen, D.Y. Youn, A.A. Sauve, Advances in characterization of human sirtuin isoforms: chemistries, targets and therapeutic applications, Curr. Med. Chem. 18 (2011) 1919-1935, https://doi.org/10.2174/092986711795590084.

[9] M. Schiedel, D. Robaa, T. Rumpf, W. Sippl, M. Jung, The current state of NAD ${ }^{+}$ dependent histone deacetylases (sirtuins) as novel therapeutic targets, Med. Res. Rev. 38 (2018) 147-200, https://doi.org/10.1002/med.21436.

[10] M. Tanno, J. Sakamoto, T. Miura, K. Shimamoto, Y. Horio, Nucleocytoplasmic shuttling of the NAD(+)-dependent histone deacetylase SIRT1, J. Biol. Chem. 282 (2007) 6823-6832, https://doi.org/10.1074/jbc.M609554200.

[11] S. Deota, T. Chattopadhyay, D. Ramachandran, E. Armstrong, B. Camacho, B. Maniyadath, A. Fulzele, A. Gonzalez-de-Peredo, J.M. Denu, U. KolthurSeetharam, Identification of a tissue-restricted isoform of SIRT1 defines a regulatory domain that encodes specificity, Cell Rep. 18 (2017) 3069-3077, https://doi.org/ 10.1016/j.celrep.2017.03.012.

[12] C.J. Lynch, Z.H. Shah, S.J. Allison, S.U. Ahmed, J. Ford, L.J. Warnock, H. Li, M. Serrano, J. Milner, SIRT1 undergoes alternative splicing in a novel auto-reg ulatory loop with p53, PLoS One 5 (2010) e13502, , https://doi.org/10.1371/ journal.pone.0013502.
[13] J.G. Rack, M.R. VanLinden, T. Lutter, R. Aasland, M. Ziegler, Constitutive nuclear localization of an alternatively spliced sirtuin-2 isoform, J. Mol. Biol. 426 (2014) 1677-1691, https://doi.org/10.1016/j.jmb.2013.10.027.

[14] Y. Yang, B.P. Hubbard, D.A. Sinclair, Q. Tong, Characterization of murine SIRT3 transcript variants and corresponding protein products, J. Cell. Biochem. 111 (2010) 1051-1058, https://doi.org/10.1002/jcb.22795.

[15] S. Bayod, J. Del Valle, J.F. Lalanza, S. Sanchez-Roige, B. de Luxán-Delgado, A. CotoMontes, A.M. Canudas, A. Camins, R.M. Escorihuela, M. Pallàs, Long-term physical exercise induces changes in sirtuin 1 pathway and oxidative parameters in adult rat tissues, Exp. Gerontol. 47 (2012) 925-935, https://doi.org/10.1016/j.exger.2012. 08.004.

[16] A. Cheng, Y. Yang, Y. Zhou, C. Maharana, D. Lu, W. Peng, Y. Liu, R. Wan, K. Marosi M. Misiak, V.A. Bohr, M.P. Mattson, Mitochondrial SIRT3 mediates adaptive responses of neurons to exercise, and metabolic and excitatory challenges, Cell Metab. 23 (2015) 128-142, https://doi.org/10.1016/j.cmet.2015.10.013.

[17] R. Garcia-Valles, M.C. Gomez-Cabrera, L. Rodriguez-Mañas, F.J. Garcia-Garcia, A. Diaz, I. Noguera, G. Olaso-Gonzalez, J. Viña, Life-long spontaneous exercise does not prolong lifespan but improves health span in mice, Longev. Healthspan 2 (2013) 14, https://doi.org/10.1186/2046-2395-2-14.

[18] A.M. Gusdon, J. Callio, J. DiStefano, R.M. O'Doherty, B.H. Goodpaster, P.M. Coen, C.T. Chu, Exercise increases mitochondrial complex I activity and DRP1 expression in the brains of aged mice, Exp. Gerontol. 90 (2017) 1-13, https://doi.org/10. 1016/j.exger.2017.01.013.

[19] J.O. Holloszy, Mortality rate and longevity of food-restricted exercising male rats: a reevaluation, J. Appl. Physiol. 82 (1997) 399-403, https://doi.org/10.1152/jappl. 1997.82.2.399.

[20] L.P. Huang, M. Yao, Y.L. Wang, A. Davie, S. Zhou, A comparison of PGC-1 $\alpha$ mRNA and protein expression in response to 1-week endurance training on alternate days or 4 consecutive days, Appl. Physiol. Nutr. Metab. 40 (2015) 1210-1213, https:// doi.org/10.1139/apnm-2015-0222.

[21] C.C. Huang, T. Wang, Y.T. Tung, W.T. Lin, Effect of exercise training on skeletal muscle SIRT1 and PGC-1 $\alpha$ expression levels in rats of different age, Int. J. Med. Sci. 13 (2016) 260-270, https://doi.org/10.7150/ijms.14586.

[22] T. Shi, F. Wang, E. Stieren, Q. Tong, SIRT3, a mitochondrial sirtuin deacetylase, regulates mitochondrial function and thermogenesis in brown adipocytes, J. Biol. Chem. 280 (2005) 13560-13567, https://doi.org/10.1074/jbc.M414670200.

[23] J.L. Steiner, E.A. Murphy, J.L. McClellan, M.D. Carmichael, J.M. Davis, Exercise training increases mitochondrial biogenesis in the brain, J. Appl. Physiol. 111 (2011) 1066-1071, https://doi.org/10.1152/japplphysiol.00343.2011.

[24] M. Tadaishi, S. Miura, Y. Kai, E. Kawasaki, K. Koshinaka, K. Kawanaka, J. Nagata, Y. Oishi, O. Ezaki, Effect of exercise intensity and AICAR on isoform-specific expressions of murine skeletal muscle PGC- $1 \alpha$ mRNA: a role of $\beta_{2}$-adrenergic receptor activation, Am. J. Physiol. Endocrinol. Metab. 300 (2011) 341-349, https://doi. org/10.1152/ajpendo.00400.2010.

[25] L. Yao, X. Cui, Q. Chen, X. Yang, F. Fang, J. Zhang, G. Liu, W. Jin, Y. Chang, Coldinducible SIRT6 regulates thermogenesis of brown and beige fat, Cell Rep. 20 (2017) 641-654, https://doi.org/10.1016/j.celrep.2017.06.069.

[26] N.A. Tritos, J.W. Mastaitis, E.G. Kokkotou, P. Puigserver, B.M. Spiegelman, E. Maratos-Flier, Characterization of the peroxisome proliferator activated receptor coactivator 1 alpha (PGC 1alpha) expression in the murine brain, Brain Res. 961 (2003) 255-260, https://doi.org/10.1016/S0006-8993(02)03961-6.

[27] X. Wen, J. Wu, J.S. Chang, P. Zhang, J. Wang, Y. Zhang, T.W. Gettys, Y. Zhang, Effect of exercise intensity on isoform-specific expressions of NT-PGC-1 $\alpha$ mRNA in mouse skeletal muscle, Biomed Res. Int. (2014) 402175, , https://doi.org/10.1155/ 2014/402175 2014

[28] Z. Gerhart-Hines, J.T. Rodgers, O. Bare, C. Lerin, S.H. Kim, R. Mostoslavsky, F.W. Alt, Z. Wu, P. Puigserver, Metabolic control of muscle mitochondrial function and fatty acid oxidation through SIRT1/PGC-1alpha, EMBO J. 26 (2007) 1913-1923, https://doi.org/10.1038/sj.emboj.7601633.

[29] J.T. Rodgers, C. Lerin, Z. Gerhart-Hines, P. Puigserver, Metabolic adaptations through the PGC-1 $\alpha$ and SIRT1 pathways, FEBS Lett. 582 (2008) 46-53, https:// doi.org/10.1016/j.febslet.2007.11.034.

[30] R.K. Chaturvedi, M.F. Beal, Mitochondrial diseases of the brain, Free Radic. Biol. Med. 63 (2013) 1-29, https://doi.org/10.1016/j.freeradbiomed.2013.03.018.

[31] J. Eschbach, B. Schwalenstöcker, S.M. Soyal, H. Bayer, D. Wiesner, C. Akimoto, A.C. Nilsson, A. Birve, T. Meyer, L. Dupuis, K.M. Danzer, P.M. Andersen, A. Witting, A.C. Ludolph, W. Patsch, P. Weydt, PGC-1 $\alpha$ is a male-specific disease modifier of human and experimental amyotrophic lateral sclerosis, Hum. Mol. Genet. 22 (2013) 3477-3484, https://doi.org/10.1093/hmg/ddt202.

[32] P. Weydt, S.M. Soyal, G.B. Landwehrmeyer, W. Patsch, European Huntington Disease Network, A single nucleotide polymorphism in the coding region of PGC-1a is a male-specific modifier of Huntington disease age-at-onset in a large European cohort, BMC Neurol. 14 (2014) 1, https://doi.org/10.1186/1471-2377-14-1.

[33] Y. Kanfi, S. Naiman, G. Amir, V. Peshti, G. Zinman, L. Nahum, Z. Bar-Joseph, H.Y. Cohen, The sirtuin SIRT6 regulates lifespan in male mice, Nature. 483 (2012) 218-221, https://doi.org/10.1038/nature10815.

[34] K.J. Livak, T.D. Schmittgen, Analysis of relative gene expression data using realtime quantitative PCR and the 2(-Delta DeltaC(T)) Method, Methods. 25 (2001) 402-408, https://doi.org/10.1006/meth.2001.1262.

[35] M. Hamer, Y. Chida, Physical activity and risk of neurodegenerative disease: a systematic review of prospective evidence, Psychol. Med. (Paris) 39 (2009) 3-11, https://doi.org/10.1017/S0033291708003681.

[36] C. Handschin, B.M. Spiegelman, The role of exercise and PGC1 $\alpha$ in inflammation and chronic disease, Nature 454 (2008) 463-469, https://doi.org/10.1038/ nature07206.

[37] R. Torok, A. Salamon, E. Sumegi, D. Zadori, G. Veres, M.F. Molnar, L. Vecsei, 
P. Klivenyi, Effect of MPTP on mRNA expression of PGC-1 $\alpha$ in mouse brain, Brain Res. 1660 (2017) 20-26, https://doi.org/10.1016/j.brainres.2017.01.032.

[38] E.K. Lucas, C.S. Reid, L.J. McMeekin, S.E. Dougherty, C.L. Floyd, R.M. Cowell, Cerebellar transcriptional alterations with Purkinje cell dysfunction and loss in mice lacking PGC-1 $\alpha$, Front. Cell. Neurosci. 8 (2015) 441, https://doi.org/10.3389/ fncel.2014.00441.

[39] E. Koltai, Z. Szabo, M. Atalay, I. Boldogh, H. Naito, S. Goto, C. Nyakas, Z. Radak,
Exercise alters SIRT1, SIRT6, NAD and NAMPT levels in skeletal muscle of aged rats, Mech. Ageing Dev. 131 (2010) 21-28, https://doi.org/10.1016/j.mad.2009. 11.002.

[40] L. E, J. Lu, J.M. Burns, R.H. Swerdlow, Effect of exercise on mouse liver and brain bioenergetic infrastructures, Exp. Physiol. 98 (2013) 207-219, https://doi.org/10. 1113/expphysiol.2012.066688. 
Research report

\title{
Effect of MPTP on mRNA expression of PGC- $1 \alpha$ in mouse brain
}

\author{
Rita Torok ${ }^{\mathrm{a}}$, Andras Salamon ${ }^{\mathrm{a}}$, Evelin Sumegi ${ }^{\mathrm{a}}$, Denes Zadori ${ }^{\mathrm{a}}$, Gabor Veres ${ }^{\mathrm{b}}$, Mate Fort Molnar ${ }^{\mathrm{a}}$, \\ Laszlo Vecsei $^{\mathrm{a}, \mathrm{b}}$, Peter Klivenyi ${ }^{\mathrm{a}, *}$ \\ ${ }^{a}$ Department of Neurology, University of Szeged, Semmelweis u. 6, H-6725 Szeged, Hungary \\ ${ }^{\mathrm{b}}$ MTA-SZTE Neuroscience Research Group of the Hungarian Academy of Sciences and University of Szeged, Semmelweis u. 6, H-6725 Szeged, Hungary
}

\section{A R T I C L E I N F O}

\section{Article history:}

Received 28 October 2016

Received in revised form 5 January 2017

Accepted 30 January 2017

Available online 2 February 2017

\section{Keywords:}

FL-PGC-1 $\alpha$

NT-PGC- $1 \alpha$

CNS-PGC- $1 \alpha$

MPTP

Preconditioning

Dopamine

\begin{abstract}
A B S T R A C T
The peroxisome proliferator-activated receptor- $\gamma(\operatorname{PPAR} \gamma)$ coactivator $1 \alpha(\mathrm{PGC}-1 \alpha)$ is a key regulator of mitochondrial biogenesis, respiration and adaptive thermogenesis. Besides the full-length protein (FLPGC- $1 \alpha$ ), several other functionally active PGC- $1 \alpha$ isoforms were identified as a result of alternative splicing (e.g., N-truncated PGC- $1 \alpha$; NT-PGC- $1 \alpha$ ) or alternative promoter usage (e.g., central nervous systemspecific PGC- $1 \alpha$ isoforms; CNS-PGC-1 $\alpha$ ). Achieving neuroprotection via CNS-targeted pharmacological stimulation is limited due to poor penetration of the blood brain barrier (BBB) by the proposed pharmaceutical agents, so preconditioning emerged as another option. The current study aimed to examine of how the expression levels of FL-, NT-, CNS- and reference PGC- $1 \alpha$ isoforms change in different brain regions following various 1-methyl-4-phenyl-1,2,3,6-tetrahydropyridine (MPTP) treatment regimens, including chronic low-dose treatment for preconditioning. Ninety minutes following the acute treatment regimen, the expression levels of FL-, NT- and CNS-PGC-1 $\alpha$ isoforms increased significantly in the striatum, cortex and cerebellum. However, this elevation diminished 7 days following the last MPTP injection in the acute treatment regimen. The chronic low-dose administration of MPTP, which did not cause significant toxic effects in light of the relatively unaltered dopamine levels, did not result in any significant change of PGC- $1 \alpha$ expression. The elevation of PGC- $1 \alpha$ levels following acute treatment may demonstrate a short-term compensatory mechanism against mitochondrial damage induced by the complex I inhibitor MPTP. However, drug-induced preconditioning by chronic low-dose MPTP seems not to induce protective responses via the PGC- $1 \alpha$ system.
\end{abstract}

(c) 2017 Elsevier B.V. All rights reserved.

\section{Introduction}

Parkinson's disease (PD) is a progressive neurodegenerative disorder characterized by the loss of dopaminergic neurons, and the presence of Lewy bodies in the substantia nigra (SN) pars compacta (Forno, 1996). Although the precise pathomechanism of PD is not fully understood, several molecular mechanisms of neuronal death were described in the pathogenesis, including mitochondrial dysfunction, energy deficit and oxidative stress (Bose and Beal, 2016). It is postulated that life-long cumulative low-dose exposure to mitochondrial toxins may contribute to the pathogenesis of certain neurodegenerative disorders (Harris and Blain, 2004). The delineation of 1-methyl-4-phenyl-1,2,3,6-tetrahydropyridine (MPTP) induced Parkinsonian symptoms yielded one of the first pieces of evidence that mitochondrial dysfunction is involved in

\footnotetext{
* Corresponding author.

E-mail address: klivenyi.peter@med.u-szeged.hu (P. Klivenyi).
}

PD pathogenesis (Forno et al., 1993). Accordingly, systemic MPTP administration has been widely used to study disease mechanisms in various in vivo animal studies (Javitch et al., 1985).

Besides environmental factors, several causative or susceptibility genes have been identified in PD, many of them having direct implications in mitochondrial dysfunction (Kalinderi et al., 2016). Peroxisome proliferator-activated receptor-gamma (PPAR $\gamma$ ) coactivator-1 alpha (PGC-1 $\alpha$ ) is one of them, which may play a role in PD pathogenesis. PGC $-1 \alpha$ is a multifunctional transcriptional coactivator of nuclear respiratory factors 1 and 2 (NRF-1, -2), estrogen-related receptors (ERRs) and PPARs amongst others, and hereby regulates mitochondrial function and biogenesis (Knutti and Kralli, 2001).

Analysis of human brain samples indicated that PD is associated with the increased methylation of PGC- $1 \alpha$ promoter and the reduced expression of PGC- $1 \alpha$ itself (Su et al., 2015) and its downstream-regulated genes in the SN of PD patients (Zheng et al., 2010). Furthermore, possible associations of PGC- $1 \alpha$ poly- 
morphisms with PD risk, age of onset and longevity were described as well (Clark et al., 2011). Reduced expression of PGC- $1 \alpha$ leads to enhanced $\alpha$-synuclein oligomerization, too (Ebrahim et al., 2010), and accordingly, overexpression of PGC- $1 \alpha$ produced neuroprotection against $\alpha$-synuclein- and rotenone-induced neurotoxicity (Zheng et al., 2010).

Several PGC- $1 \alpha$ isoforms were identified as a result of alternative splicing and alternative promoter usage (Martinez-Redondo et al., 2015). The proximal promoter of PGC- $1 \alpha$ has been reported as an important key regulator in several neurodegenerative diseases, including PD (Su et al., 2015). With regard to alternative splicing, besides the full-length protein (FL-PGC-1 $\alpha$ ), the N-truncated PGC- $1 \alpha$ (NT-PGC- $1 \alpha$ ) isoform was discovered, which is a shorter, but active isoform of PGC- $1 \alpha$ (Zhang et al., 2009). Recent studies identified further different tissue-specific isoforms of PGC- $1 \alpha$, including central nervous system-specific isoforms (CNS-PGC-1 $\alpha$ (Ruas et al., 2012; Soyal et al., 2012)). The novel CNS-specific isoforms originated from a new promoter located $587 \mathrm{~kb}$ upstream of exon 2 (Choi et al., 2013; Soyal et al., 2012). A recent study demonstrated that both PGC- $1 \alpha$ reference gene and CNS-PGC- $1 \alpha$ are downregulated in human PD brain and in experimental models with $\alpha$-synuclein oligomerization, and that the pharmacological activation or genetic overexpression of PGC- $1 \alpha$ reference gene reduced $\alpha$-synuclein oligomerization and toxicity (Eschbach et al., 2015). In contrast, the loss of PGC-1 $\alpha$ enhances the vulnerability of SN pars compacta dopaminergic neurons to $\alpha$-synuclein toxicity (Ciron et al., 2015). These data suggest that PGC- $1 \alpha$ downregulation and $\alpha$-synuclein oligomerization form a vicious circle (Eschbach et al., 2015). Similarly to PD, certain mutations in amyotrophic lateral sclerosis inhibit the expression of CNS-specific isoforms, indicating this as a common finding in neurodegeneration (Bayer et al., 2017).

St-Pierre et al. described that PGC-1 $\alpha$-deficient mice are more sensitive to MPTP toxicity compared to the controls (St-Pierre et al., 2006). Interestingly, the sub-chronic administration of MPTP to mice resulted in the significant elevation of PGC- $1 \alpha$ expression in the striatum after $24 \mathrm{~h}$ that was normalized following $72 \mathrm{~h}$ (Swanson et al., 2013). This may represent an adaptive mechanism to neurotoxicity. Accordingly, the protective effect of PGC- $1 \alpha$ was demonstrated previously as well; pioglitazone- and resveratrolinduced activation of PGC- $1 \alpha$ was protective against MPTP toxicity (Breidert et al., 2002; Dehmer et al., 2004). However, there is a seeming controversy with regard to the effect of geneticallyinduced overexpression of PGC- $1 \alpha$ on MPTP neurotoxicity. On the one hand, the transgenic overexpression of PGC- $1 \alpha$ was proven to be protective against MPTP (Mudo et al., 2012), on the other hand, the adenovirus vector-mediated overexpression of PGC- $1 \alpha$ resulted in dopamine depletion in the SN (Ciron et al., 2012) and consequently enhanced susceptibility to MPTP (Clark et al., 2012). Clarification of this issue needs further studies.

Evidence suggests a beneficial role of PGC- $1 \alpha$ stimulation in neurodegenerative disorders. However, CNS-targeted pharmacological stimulation is limited due to the poor penetration of the blood brain barrier (BBB) by the above-mentioned compounds, so preconditioning emerged as another option to achieve neuroprotection. It was previously demonstrated that the acute administration of the selective complex II inhibitor 3-nitropropionic acid (3NP) increased the expression of both FL- and NT-PGC- $1 \alpha$ isoforms in the striatum of C57Bl/6 mice (Torok et al., 2015). As the available data are limited with regard to the alteration of tissue-specific PGC $-1 \alpha$ expression in the brain following MPTP administration, this study aimed to examine the expression levels of several PGC- $1 \alpha$ isoforms in different brain regions following various MPTP treatment regimens. The hypothesis that low doses of MPTP may produce compensatory, protective alterations in the PGC- $1 \alpha$ system was tested as well.

\section{Results}

\subsection{Gene expression analysis}

Ninety minutes following the last MPTP injection of the acute treatment of MPTP, the FL-PGC- $1 \alpha$ and NT-PGC- $1 \alpha$ expression significantly increased in the striatum (FL-PGC-1 $\alpha$ : ctrl: 0.97 (0.92-1.04), MPTP: 1.47 (1.21-1.83), p = 0.0048; NT-PGC-1 $\alpha$ : ctrl: 0.44 (0.40-0.49), MPTP: $0.70(0.56-0.78), \mathrm{p}=0.019)$, cortex (FL-PGC-1 $\alpha$ : ctrl: 0.96 (0.91-1.06), MPTP: 1.23 (1.15-1.43), $\mathrm{p}=0.009 ; \quad$ NT-PGC- $1 \alpha$ : ctrl: 0.46 (0.43-0.48), MPTP: 0.69 (0.59-0.71), $\mathrm{p}=0.0012$ ) and cerebellum (FL-PGC-1 $\alpha$ : ctrl: 1.50 (1.27-1.90), MPTP: 2.40 (2.07-2.76), p=0.013; NT-PGC-1 $\alpha$ : ctrl: 0.67 (0.48-0.86), MPTP: 1.21 (1.14-1.44), p=0.009) (Fig. 1A, B). Furthermore, MPTP-induced increases in CNS-PGC- $1 \alpha$ expression were also significantly larger in all investigated brain regions compared to the controls (striatum: ctrl: 1.03 (0.88-1.11), MPTP: 1.38 (1.34-1.78), p=0.0069; cortex: ctrl: 0.91 (0.80-0.98), МPTP: 1.41 (1.24-1.42), $\mathrm{p}=0.0048$; cerebellum: ctrl: 1.51 (1.20-1.98), MPTP: 2.77 (2.34-3.17), $\mathrm{p}=0.019$ ) (Fig. 1C). However, there was not any difference between the Ref-PGC- $1 \alpha$ levels in the striatum (ctrl: 0.11 (0.10-0.12), MPTP: 0.11 (0.95-0.12)), cortex (ctrl: 0.11 (0.11-0.12), MPTP: 0.09 (0.08-0.10)) and cerebellum (ctrl: $0.21(0.20-0.29)$, MPTP: 0.28 (0.24-0.29)) of MPTP-treated and control mice (Fig. 1D).

One week following the last injection in the acute treatment regimen, there was not any significant change either in the FL-, NT-, CNS-, or in the Ref-PGC- $1 \alpha$ levels between the control and the MPTP-treated animals in any brain area (Fig. 2A-D).

Furthermore, the low-dose 12-day MPTP-treatment did not influence the expression levels of FL-PGC-1 $\alpha$, NT-PGC-1 $\alpha$, CNS-PGC- $1 \alpha$ and Ref-PGC- $1 \alpha$ in any brain region (Fig. 3A-D).

\subsection{HPLC measurement}

Dopamine (DA), 3,4-dihydroxyphenylacetic acid (DOPAC) and homovanillic acid (HVA) values in the respective control groups of the 3 treatment regimens were compared to each other, and there were no significant differences. Therefore the values in these control groups were pooled for further comparisons with the MPTP-treated groups. MPTP administration caused significant reductions in striatal DA (ctrl: $8.08 \pm 0.50$, MPTP: $4.36 \pm 0.92$, $\mathrm{p}=0.0005$ ), DOPAC (ctrl: $2.57 \pm 0.21$, MPTP: $0.44 \pm 0.08$, $\mathrm{p}=3.78^{*} 10^{-8}$ ) and HVA (ctrl: $2.18 \pm 0.12$, MPTP $0.67 \pm 0.11$, $\mathrm{p}=5.12^{*} 10^{-10}$ ) levels compared to control values 90 min following its last administration in the acute treatment regimen (acute1 day; Fig. 4). Moreover, a significant reduction in metabolite levels was also observed one week after the last injection in the acute treatment regimen (acute-7 days; Fig. 4) in the DA (ctrl: $8.08 \pm 0.50, \quad$ MPTP: $1.34 \pm 0.43, \quad \mathrm{p}=4.86^{*} 10^{-8}$ ), DOPAC (ctrl: $2.57 \pm 0.21$, MPTP: $0.76 \pm 0.15, \quad \mathrm{p}=7^{*} 10^{-6}$ ) and HVA (ctrl: $2.18 \pm 0.12$, MPTP: $0.81 \pm 0.13, \mathrm{p}=5.08^{*} 10^{-8}$ ) values in the striatum of the MPTP-treated mice compared to the control animals. However, chronic MPTP treatment resulted in significant reductions of only striatal HVA (ctrl: $2.18 \pm 0.12$, MPTP $1.40 \pm 0.08$, $\mathrm{p}=0.0005$ ) levels, striatal DA (ctrl: 8.08 \pm 0.50 , MPTP: $6.83 \pm 0.48)$ and DOPAC (ctrl: $2.57 \pm 0.21$; MPTP $1.99 \pm 0.23$ ) levels were not decreased significantly (Fig. 4). Seven days following the acute treatment regimen DA levels significantly decreased compared to those data from samples obtained 90 min following the last MPTP injection in the acute treatment regimen $(\mathrm{p}=0.039)$.

\section{Discussion}

PGC $-1 \alpha$ is essential in normal mitochondrial function and its deficiency may contribute to neurodegeneration, while its stimula- 
A

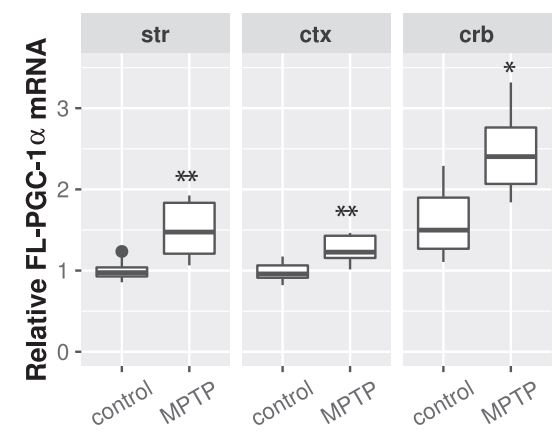

C

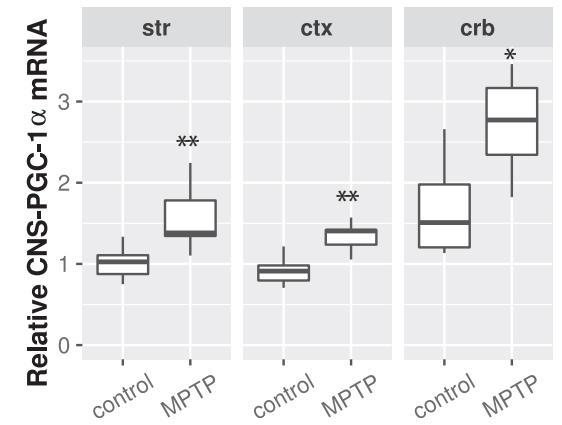

B

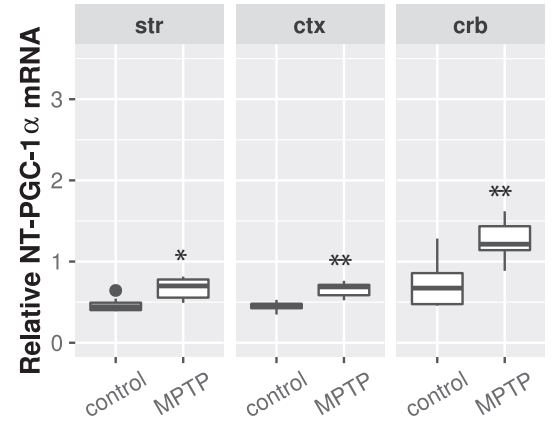

D

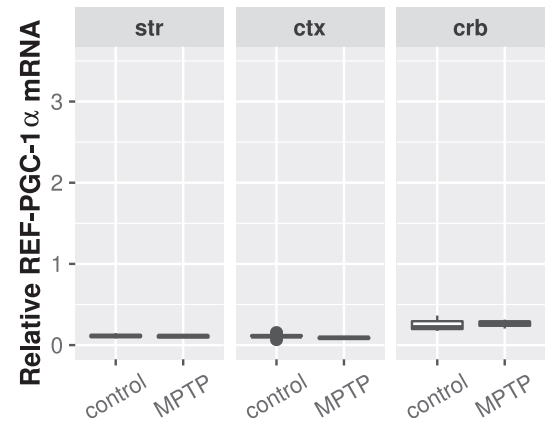

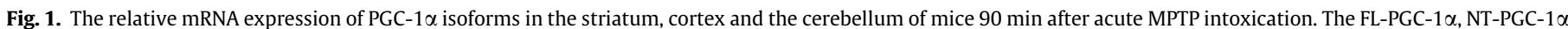

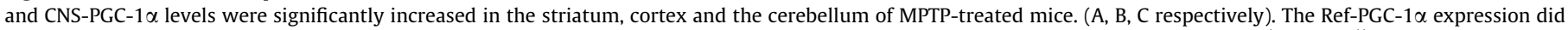

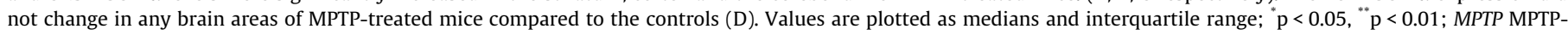
treated; str striatum, $c t x$ cortex, $c r b$ cerebellum.

A

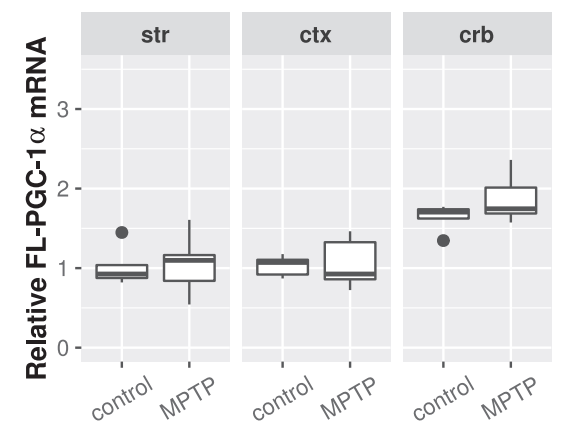

C

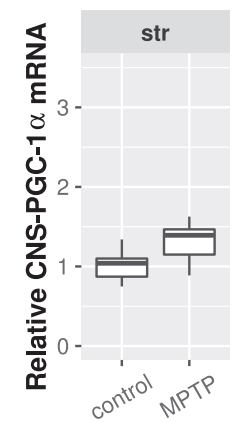

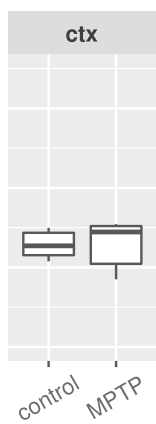

B

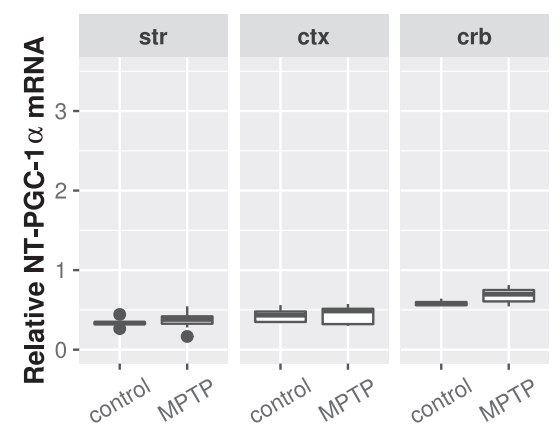

D

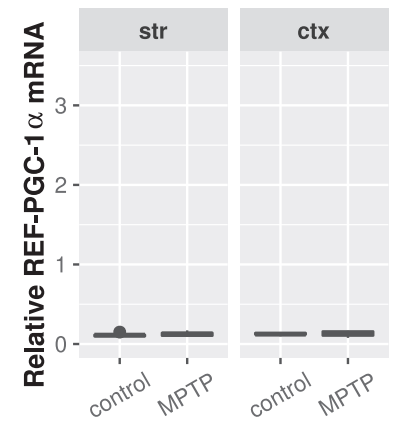

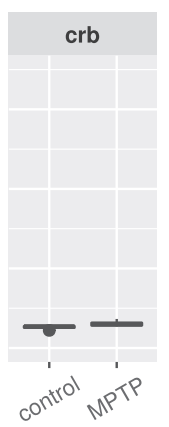

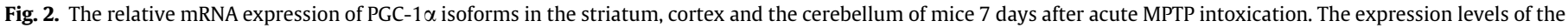

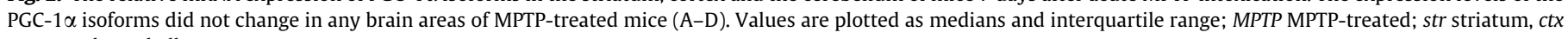
cortex, crb cerebellum.

tion was demonstrated to be neuroprotective in certain models (Breidert et al., 2002; Dehmer et al., 2004; Eschbach et al., 2015; Mudo et al., 2012). Accordingly, the pharmacological induction of PGC-1 $\alpha$ expression may be considered as a neuroprotective approach, but currently this possibility seems to be limited in light of the reduced BBB penetration of the potential pharmaceutical agents.

The aim of the current study was a thorough assessment of the expression of PGC- $1 \alpha$ isoforms in various brain regions following different MPTP administration regimens, including a 
A

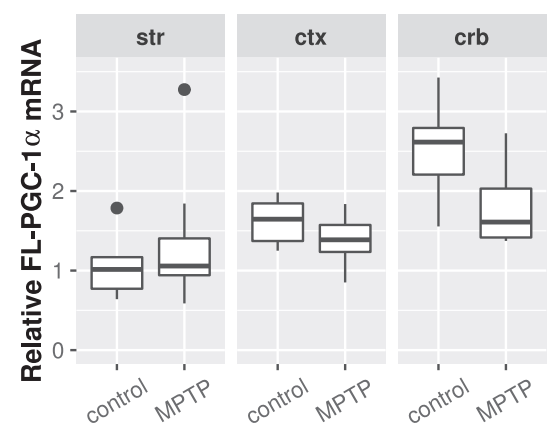

C

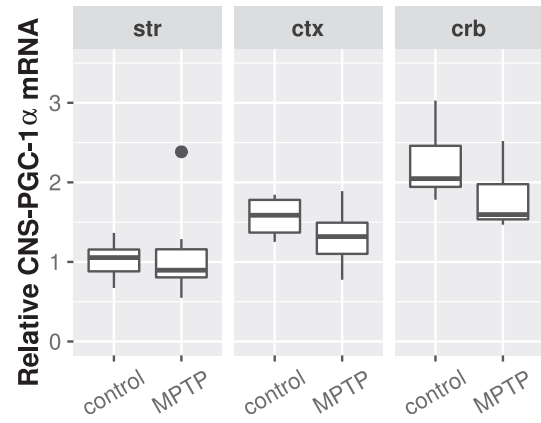

B

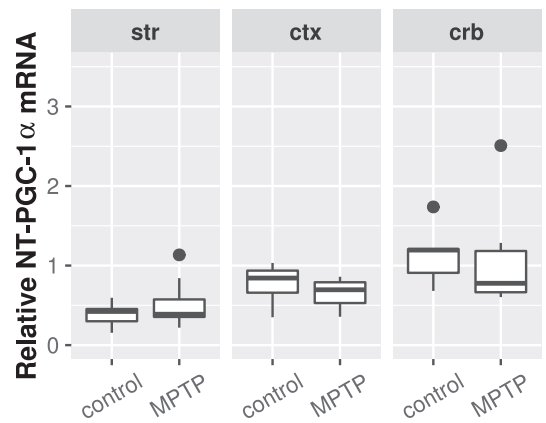

D

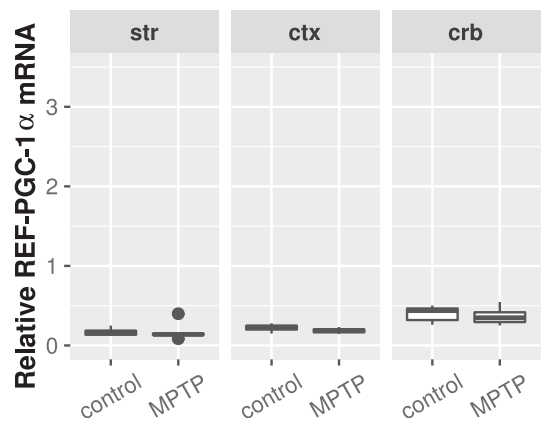

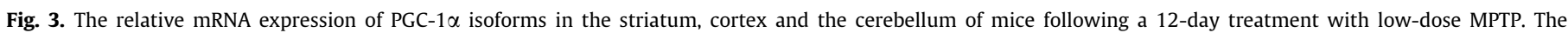

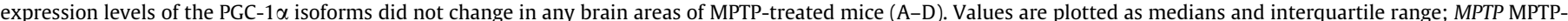
treated; str striatum, $c t x$ cortex, $c r b$ cerebellum.

low-dose chronic one, possibly mimicking drug-induced preconditioning.

Ninety minutes following the last MPTP injection of the highdose acute treatment regimen of MPTP ( $75 \mathrm{mg} / \mathrm{kg} /$ day total dose) the expression level of FL-, NT- and CNS- PGC- $1 \alpha$ isoforms increased significantly in the striatum, cortex and cerebellum. However, this elevation was diminished 7 days following the last MPTP injection in the acute treatment regimen. Torok et al. (Torok et al., 2015) demonstrated that the acute (90 min following a single dose injection of $100 \mathrm{mg} / \mathrm{kg}$ dose), but not the subacute (50 $\mathrm{mg} / \mathrm{kg}$ twice daily for 5 days) 3-NP treatment regimen induced the overexpression of FL- and NT- PGC- $1 \alpha$ isoforms mainly in the striatum (3-NP is a rather selective striatal neurotoxin (Brouillet et al., 2005)) similar to the results of the current study. Those findings were explained by a proposed reduced neuronal adaptive capability of the striatum following the neurotoxic insult. The above-mentioned results of the current study may also be explained by the propagation of the neurotoxic process following 7 days of the acute treatment regimen given the extent of decrease in striatal DA levels. The elevation of PGC- $1 \alpha$ expression, especially that of the CNS-specific isoform, may indicate a short-term compensatory protective mechanism against mitochondrial dysfunction induced by the complex I inhibitor MPTP. It is hard to interpret the increased expression of PGC- $1 \alpha$ in the cerebellum, which is not primarily affected in MPTP toxicity. However, several lines of evidence indicate that MPTP neurotoxicity is not highly selective to dopaminergic neurons; in specific circumstances systemic MPTP administration resulted in Purkinje cell loss (Takada et al., 1993). The involvement of the cerebellum in disease mechanisms of different neurodegenerative disorders such as amyotrophic lateral sclerosis, Huntington's disease (HD) and PD is frequently seen (Rees et al., 2014; Tan et al., 2016; Wu and Hallett, 2013). Furthermore, increasing evidence suggest that PGC $-1 \alpha$ expression is associated with degenerative changes in the CNS, including cerebellum (Torok et al., 2015). It was hypothe- sized that the elevation of PGC- $1 \alpha$ in the cerebellum is a compensatory mechanism against the energy deficit which may be an important factor underlying the relative resistance of cerebellar neurons against neurodegenerative processes in HD and in PD.

The drug-induced preconditioning by applying low-dose neurotoxic agents may stimulate neuroprotective mechanisms, resulting in the amelioration of neurodegenerative process. This approach has already been demonstrated to be beneficial in case of 3-NP: the low-dose of toxin treatment increased tolerance to ischemia and hypoxia in rats and gerbils (Horiguchi et al., 2003; Riepe et al., 1997; Wiegand et al., 1999). Although the exact mechanism is not fully understood, the overexpression of free radical scavenging enzymes may be involved: acute 3-NP treatment activated superoxide dismutase (SOD) and catalase (CAT) in several brain areas (Binienda et al., 1998). Similarly, an increase in SOD activity in the glial cells of the striatum and SN was observed following MPTP treatment (Kurosaki et al., 2004). The preconditioning by MPTP is not intended to suggest a future direct therapeutic approach, but rather aimed at finding key players which may help to alleviate the pathological alterations. The situation may be similar to ischemic preconditioning where the outcome in myocardial infarction may depend substantially on which medications were applied with an influence on preconditioning (Tomai et al., 1999). This may be especially important in light of the fact that environmental toxins could play a role in the pathogenies of idiopathic PD. The chronic low-dose administration of MPTP in the current study neither resulted in significant DA depletion (i.e. neurotoxic effect at biochemical level), nor in any significant change in PGC- $1 \alpha$ expression. These data suggest that druginduced preconditioning by MPTP may not evoke apparent responses in the PGC- $1 \alpha$ system.

In conclusion the current study demonstrated that acute severe mitochondrial dysfunction initiated protection via elevating the expression of brain specific isoforms of the mitochondrial master regulator PGC- $1 \alpha$. However, low-dose chronic administration of 


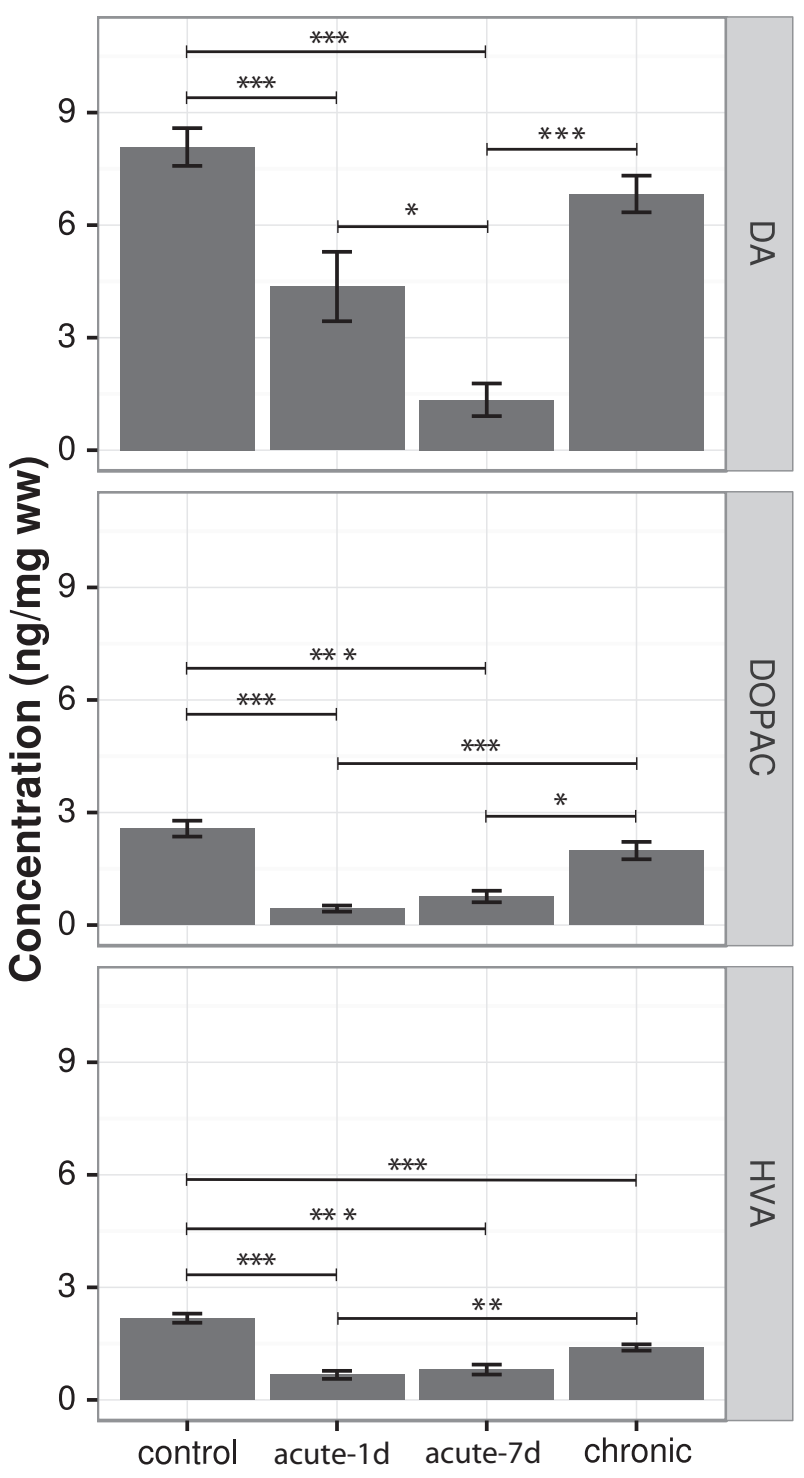

Fig. 4. Striatal dopamine, DOPAC and HVA concentrations of MPTP-treated mice in 3 different treatment regimens. Ninety minutes (acute-1d) and 7 days (acute-7d) after acute MPTP intoxication, DA, DOPAC and HVA levels significantly decreased in the striatum compared to the controls. The chronic (12 day) low-dose MPTP treatment did not influence the striatal level of DA and DOPAC, only HVA levels were significantly decreased. Values are plotted as means \pm S.E.M; ${ }^{* * *} \mathrm{p}<0.001 ; D A$ dopamine, DOPAC 3,4-dihydroxyphenylacetic acid, HVA homovanillic acid.

mitochondrial toxin MPTP did not induce those protective mechanisms with the involvement of PGC- $1 \alpha$.

\section{Experimental procedures}

\subsection{Animals}

12-Week-old C57Bl/6J male mice were used in this study. The animal strain was originally obtained from Jackson Labs (Jackson Laboratories, Bar Harbor, ME, USA).

The animals were housed in cages and maintained under standard laboratory conditions with 12-12 h light-dark cycle and free access to food and water. The experiments were carried out in accordance with the European Communities Council Directive $(86 / 609 / \mathrm{EEC})$ and were approved by the local animal care committee.

\subsection{Treatment and sample handling}

MPTP was dissolved in phosphate-buffered saline (PBS; $\mathrm{pH}$ adjusted to 7.4) and was administered intraperitoneally (i.p.). Animals were randomly divided into six groups $(n=7-8$ in each group). The first and second group received i.p. injection of $15 \mathrm{mg} / \mathrm{kg}$ body weight MPTP 5 times at $2 \mathrm{~h}$ intervals. The animals in the first group were deeply anesthetized with isoflurane (Forane; Abott Laboratories Hungary Ltd., Budapest, Hungary) and the brains were dissected ninety minutes following the last MPTP injection (acute treatment - acute (day 1 ) assessment), while animals in the second group were deeply anesthetized with isoflurane and the brains were dissected one week later (acute treatment subacute (day 7) assessment). The mice in the third group were injected i.p. with $15 \mathrm{mg} / \mathrm{kg}$ body weight MPTP once a day for 12 days (low-dose chronic treatment). Ninety minutes following the last injection the animals were euthanized via isoflurane overdose as well. The fourth, fifth and sixth groups served as the respective control groups, and were injected with $0.1 \mathrm{M}$ PBS according to the above-detailed treatment regimen. During the dissection process the brains were rapidly removed on ice and immediately halved at the midline. Following that, both hemispheres were further cut to obtain the striatum, cortex and cerebellum. Thereafter, these samples were stored at $-80^{\circ} \mathrm{C}$ until the RT-PCR and HPLC analysis.

\subsection{RT-PCR analysis}

The left striatum, cortex and cerebellum were homogenized and Trizol reagent was used to extract RNA according to the manufacturer's protocol. The RNA was quantified spectrophotometrically, and the integrity of RNA was confirmed by gel electrophoresis using $1 \%$ agarose gel. $1 \mu \mathrm{g}$ of total RNA was reverse-transcribed applying random hexamer primers and reverse transcriptase according to the RevertAid First Strand cDNA Synthesis Kit protocol (Thermo Fisher Scientific Inc., Marietta, OH, USA). cDNAs were kept at $-20^{\circ} \mathrm{C}$ until further use.

Real-time PCR reactions were carried out in a $20 \mu \mathrm{l}$ final volume.

The following, previously published primers were used: for FLPGC- $1 \alpha, 5^{\prime}$-TGCCATTGTTAAGACCGAG-3' (forward) and 5'-TTGGG GTCATTTGGTGAC-3' (reverse); for NT-PGC-1 $\alpha, 5^{\prime}$-GGTCACTGGAA GATATGGC-3' (reverse); for CNS-PGC- $1 \alpha$ and Ref-PGC- $1 \alpha, 5^{\prime}$-AAT TGGAGCCCCATGGATGAAGG- $3^{\prime}$ and $5^{\prime}$-TGAGTCTGTATGGAGTGA CATCGAGTG-3' (both forward), and 5'-TCAAATGAGGG CAATCCGTC-3' (reverse), respectively (Chang et al., 2012; Soyal et al., 2012). qRT-PCR reaction conditions were $95^{\circ} \mathrm{C}$ for $2 \mathrm{~min}$, followed by 40 cycles of $95^{\circ} \mathrm{C}$ for $10 \mathrm{~s}$, and $60^{\circ} \mathrm{C}$ for $30 \mathrm{~s}$. Target gene expression was normalized to the endogenous control gene $18 \mathrm{~S}$ rRNA (Applied Biosystems, Carlsbad, CA, USA). The relative expression was determined using the $2^{-\Delta \Delta C t}$ method (Livak and Schmittgen, 2001).

\subsection{Dopamine measurement}

DA and its metabolites, DOPAC and HVA were measured by reversed-phase chromatography from the right striatum of the MPTP-treated and the control animals, using an Agilent 1100 high-performance liquid chromatography (HPLC) system (Agilent Technologies, Santa Clara, CA, USA) combined with a Model 105 electrochemical detector (Precision Instruments, Marseille, France) under isocratic conditions. The striata were weighed and then homogenized in an ice-cold solution $(750 \mu \mathrm{l})$ containing perchloric acid $(70 \% \mathrm{wt} / \mathrm{wt})$, sodium metabisulfite $(0.1 \mathrm{M})$, disodium ethylenediaminetetraacetate $(0.1 \mathrm{M})$, distilled water and $0.25 \mathrm{mM}$ isoproterenol for $30 \mathrm{~s}$. The homogenate was centrifuged at 
$12,000 \mathrm{~g}$ for $10 \mathrm{~min}$ at $4^{\circ} \mathrm{C}$. The supernatant was stored at $-20^{\circ} \mathrm{C}$ until the analysis. The supernatants were measured with an Agilent 1100 high-performance liquid chromatography (HPLC) system (Agilent Technologies, Santa Clara, CA, USA) combined with a Model 105 electrochemical detector (Precision Instruments, Marseille, France) under isocratic conditions. In brief, the working potential of the detector was set at $+750 \mathrm{mV}$, using a glassy carbon electrode and a $\mathrm{Ag} / \mathrm{AgCl}$ reference electrode. The mobile phase containing sodium dihydrogenphosphate $(75 \mathrm{mM})$, sodium octylsulfate $(2.8 \mathrm{mM})$ and disodium ethylenediaminetetraacetate $(50 \mu \mathrm{M})$ was supplemented with acetonitrile $(10 \% \mathrm{v} / \mathrm{v})$ and the $\mathrm{pH}$ was adjusted to 3 with phosphoric acid $(85 \% \mathrm{w} / \mathrm{w})$. The mobile phase was delivered at a rate of $1 \mathrm{ml} / \mathrm{min}$ at $40^{\circ} \mathrm{C}$ onto the reversedphase column (HR-80 C18, $80 \times 4.6 \mathrm{~mm}, 3 \mu \mathrm{m}$ particle size; ESA Biosciences, Chelmsford, MA, USA) after passage through a precolumn (SecurityGuard, $4 \times 3.0 \mathrm{~mm}$ I.D., $5 \mu \mathrm{m}$ particle size, Phenomenex Inc., Torrance, CA, USA)). $10 \mu$ l aliquots were injected by the autosampler with the cooling module set at $4^{\circ} \mathrm{C}$. With regard to method validation, the following parameters are reported briefly. The LOD and LLOQ for the investigated compounds in the brain samples were $2 \mathrm{ng} / \mathrm{ml}$ and $10 \mathrm{ng} / \mathrm{ml}$, respectively. With regard to precision, the relative standard deviation was $\leqslant 3.25 \%$ for the peak area responses and $\leqslant 0.05 \%$ for the retention times. The recoveries ranged from 109 to $110 \%, 108$ to $109 \%$ and 99 to $102 \%$ for DA, DOPAC and HVA, respectively.

\subsection{Statistics}

All statistical analyses were performed with the use of the $R$ software (R Development Core Team). The distribution of data populations was checked with the Shapiro-Wilk test, and Levene test was also performed for the analysis of the homogeneity of variances. In case of gene expression analysis, due to the necessity of a large number of comparisons of data, two-sample t-tests via Monte-Carlo permutation (with 10,000 random permutations) were applied for RT-PCR results. In case of HPLC analysis, all the data exhibited normal distribution and equal variances were assumed, and therefore ANOVA was used with Bonferroni post hoc comparison. The null hypothesis was rejected when the corrected $p$ values were $<0.05$, and in such cases the differences were considered significant. FL- and NT-PGC- $1 \alpha$ levels of gene expression of all brain areas were calculated relative to the levels of FLPGC- $1 \alpha$ gene expression in the striatum, whereas the CNS- and Ref-PGC- $1 \alpha$ expression levels of all brain areas were calculated relative to the level of CNS-PGC- $1 \alpha$ expression in the striatum. Data with Gaussian or non-Gaussian distributions were plotted as means ( \pm S.E.M.) or medians (and interquartile range), respectively.

\section{Funding sources}

The study was supported by the Hungarian Brain Research Program - Grant No. KTIA_13_NAP-A-II/18 and MTA-SZTE Neuroscience Research Group. Denes Zadori was supported by the Janos Bolyai Research Scholarship of the Hungarian Academy of Sciences.

\section{Conflict of interest}

The authors declare there is no conflict of interest.

\section{References}

Bayer, H., Lang, K., Buck, E., Higelin, J., Barteczko, L., Pasquarelli, N., Sprissler, J. Lucas, T., Holzmann, K., Demestre, M., Lindenberg, K.S., Danzer, K.M., Boeckers, T., Ludolph, A.C., Dupuis, L., Weydt, P., Witting, A., 2017. ALS-causing mutations differentially affect PGC-1alpha expression and function in the brain vs. peripheral tissues. Neurobiol. Dis. 97, 36-45.

Binienda, Z., Simmons, C., Hussain, S., Slikker Jr., W., Ali, S.F., 1998. Effect of acute exposure to 3-nitropropionic acid on activities of endogenous antioxidants in the rat brain. Neurosci. Lett. 251, 173-176.

Bose, A., Beal, M.F., 2016. Mitochondrial dysfunction in Parkinson's disease. J. Neurochem. 139 (Suppl 1), 216-231.

Breidert, T., Callebert, J., Heneka, M.T., Landreth, G., Launay, J.M., Hirsch, E.C., 2002. Protective action of the peroxisome proliferator-activated receptor-gamma agonist pioglitazone in a mouse model of Parkinson's disease. J. Neurochem. 82, 615-624.

Brouillet, E., Jacquard, C., Bizat, N., Blum, D., 2005. 3-Nitropropionic acid: a mitochondrial toxin to uncover physiopathological mechanisms underlying striatal degeneration in Huntington's disease. J. Neurochem. 95, 1521-1540.

Chang, J.S., Fernand, V., Zhang, Y., Shin, J., Jun, H.J., Joshi, Y., Gettys, T.W., 2012. NTPGC-1alpha protein is sufficient to link beta3-adrenergic receptor activation to transcriptional and physiological components of adaptive thermogenesis. J. Biol. Chem. 287, 9100-9111.

Choi, J., Batchu, V.V., Schubert, M., Castellani, R.J., Russell, J.W., 2013. A novel PGC1alpha isoform in brain localizes to mitochondria and associates with PINK1 and VDAC. Biochem. Biophys. Res. Commun. 435, 671-677.

Ciron, C., Lengacher, S., Dusonchet, J., Aebischer, P., Schneider, B.L., 2012. Sustained expression of PGC-1alpha in the rat nigrostriatal system selectively impairs dopaminergic function. Hum. Mol. Genet. 21, 1861-1876.

Ciron, C., Zheng, L., Bobela, W., Knott, G.W., Leone, T.C., Kelly, D.P., Schneider, B.L., 2015. PGC-1alpha activity in nigral dopamine neurons determines vulnerability to alpha-synuclein. Acta Neuropathol. Commun. 3, 16.

Clark, J., Reddy, S., Zheng, K., Betensky, R.A., Simon, D.K., 2011. Association of PGC1alpha polymorphisms with age of onset and risk of Parkinson's disease. BMC Med. Genet. 12, 69.

Clark, J., Silvaggi, J.M., Kiselak, T., Zheng, K., Clore, E.L., Dai, Y., Bass, C.E., Simon, D.K., 2012. Pgc-1alpha overexpression downregulates Pitx3 and increases susceptibility to MPTP toxicity associated with decreased Bdnf. PLoS ONE 7, e48925.

Dehmer, T., Heneka, M.T., Sastre, M., Dichgans, J., Schulz, J.B., 2004. Protection by pioglitazone in the MPTP model of Parkinson's disease correlates with I kappa B alpha induction and block of NF kappa B and iNOS activation. J. Neurochem. 88, 494-501.

Ebrahim, A.S., Ko, L.W., Yen, S.H., 2010. Reduced expression of peroxisomeproliferator activated receptor gamma coactivator-1alpha enhances alphasynuclein oligomerization and down regulates AKT/GSK3beta signaling pathway in human neuronal cells that inducibly express alpha-synuclein. Neurosci. Lett. 473, 120-125.

Eschbach, J., von Einem, B., Muller, K., Bayer, H., Scheffold, A., Morrison, B.E., Rudolph, K.L., Thal, D.R., Witting, A., Weydt, P., Otto, M., Fauler, M., Liss, B., McLean, P.J., Spada, A.R., Ludolph, A.C., Weishaupt, J.H., Danzer, K.M., 2015. Mutual exacerbation of peroxisome proliferator-activated receptor gamma coactivator 1alpha deregulation and alpha-synuclein oligomerization. Ann. Neurol. 77, 15-32.

Forno, L.S., DeLanney, L.E., Irwin, I., Langston, J.W., 1993. Similarities and differences between MPTP-induced parkinsonsim and Parkinson's disease. Neuropathologic considerations. Adv. Neurol. 60, 600-608.

Forno, L.S., 1996. Neuropathology of Parkinson's disease. J. Neuropathol. Exp. Neurol. 55, 259-272.

Harris, J.B., Blain, P.G., 2004. Neurotoxicology: what the neurologist needs to know. J. Neurol. Neurosurg. Psychiatry 75 (Suppl 3), iii29-iii34.

Horiguchi, T., Kis, B., Rajapakse, N., Shimizu, K., Busija, D.W., 2003. Opening of mitochondrial ATP-sensitive potassium channels is a trigger of 3-nitropropionic acid-induced tolerance to transient focal cerebral ischemia in rats. Stroke 34, 1015-1020.

Javitch, J.A. D'Amato, R.J. Strittmatter, S.M. Snyder, S.H., 1985. Parkinsonisminducing neurotoxin, $\mathrm{N}$-methyl-4-phenyl-1,2,3,6 -tetrahydropyridine: uptake of the metabolite $\mathrm{N}$-methyl-4-phenylpyridine by dopamine neurons explains selective toxicity. Proc. Natl. Acad. Sci. U.S.A. 82, 2173-2177.

Kalinderi, K., Bostantjopoulou, S., Fidani, L., 2016. The genetic background of Parkinson's disease: current progress and future prospects. Acta Neurol. Scand. $134,314-326$.

Knutti, D., Kralli, A., 2001. PGC-1, a versatile coactivator. Trends Endocrinol. Metab. 12, 360-365.

Kurosaki, R., Muramatsu, Y., Kato, H., Araki, T., 2004. Biochemical, behavioral and immunohistochemical alterations in MPTP-treated mouse model of Parkinson's disease. Pharmacol. Biochem. Behav. 78, 143-153.

Livak, K.J., Schmittgen, T.D., 2001. Analysis of relative gene expression data using real-time quantitative PCR and the 2(-Delta Delta C(T)) Method. Methods 25, 402-408.

Martinez-Redondo, V., Pettersson, A.T., Ruas, J.L., 2015. The hitchhiker's guide to PGC-1alpha isoform structure and biological functions. Diabetologia 58, 19691977.

Mudo, G., Makela, J., Di Liberto, V., Tselykh, T.V., Olivieri, M., Piepponen, P., Eriksson, O., Malkia, A., Bonomo, A., Kairisalo, M., Aguirre, J.A., Korhonen, L., Belluardo, N., Lindholm, D., 2012. Transgenic expression and activation of PGC-1alpha protect dopaminergic neurons in the MPTP mouse model of Parkinson's disease. Cell. Mol. Life Sci. 69, 1153-1165.

Rees, E.M., Farmer, R., Cole, J.H., Haider, S., Durr, A., Landwehrmeyer, B., Scahill, R.I., Tabrizi, S.J., Hobbs, N.Z., 2014. Cerebellar abnormalities in Huntington's disease: a role in motor and psychiatric impairment? Mov. Disord. 29, 1648-1654. 
Riepe, M.W., Esclaire, F., Kasischke, K., Schreiber, S., Nakase, H., Kempski, O., Ludolph, A.C., Dirnagl, U., Hugon, J., 1997. Increased hypoxic tolerance by chemical inhibition of oxidative phosphorylation: "chemical preconditioning". J. Cereb. Blood Flow Metab. 17, 257-264.

Ruas, J.L., White, J.P., Rao, R.R., Kleiner, S., Brannan, K.T., Harrison, B.C., Greene, N.P., Wu, J., Estall, J.L., Irving, B.A., Lanza, I.R., Rasbach, K.A., Okutsu, M., Nair, K.S., Yan, Z., Leinwand, L.A., Spiegelman, B.M., 2012. A PGC-1alpha isoform induced by resistance training regulates skeletal muscle hypertrophy. Cell 151, 1319-1331.

Soyal, S.M., Felder, T.K., Auer, S., Hahne, P., Oberkofler, H., Witting, A., Paulmichl, M., Landwehrmeyer, G.B., Weydt, P., Patsch, W.European Huntington Disease Network, 2012. A greatly extended PPARGC1A genomic locus encodes several new brain-specific isoforms and influences Huntington disease age of onset. Hum. Mol. Genet. 21, 3461-3473.

St-Pierre, J., Drori, S., Uldry, M., Silvaggi, J.M., Rhee, J., Jager, S., Handschin, C., Zheng K., Lin, J., Yang, W., Simon, D.K., Bachoo, R., Spiegelman, B.M., 2006. Suppression of reactive oxygen species and neurodegeneration by the PGC-1 transcriptional coactivators. Cell 127, 397-408.

Su, X., Chu, Y., Kordower, J.H., Li, B., Cao, H., Huang, L., Nishida, M., Song, L., Wang, D., Federoff, H.J., 2015. PGC-1alpha promoter methylation in Parkinson's disease. PLOS ONE 10, e0134087.

Swanson, C.R., Du, E., Johnson, D.A., Johnson, J.A., Emborg, M.E., 2013. Neuroprotective properties of a novel non-thiazoledinedione partial PPARgamma agonist against MPTP. PPAR Res. 2013, 582809.

Takada, M., Sugimoto, T., Hattori, T., 1993. MPTP neurotoxicity to cerebellar Purkinje cells in mice. Neurosci. Lett. 150, 49-52.
Tan, R.H., Kril, J.J., McGinley, C., Hassani, M., Masuda-Suzukake, M., Hasegawa, M. Mito, R., Kiernan, M.C., Halliday, G.M., 2016. Cerebellar neuronal loss in amyotrophic lateral sclerosis cases with ATXN2 intermediate repeat expansions. Ann. Neurol. 79, 295-305.

Tomai, F. Crea, F. Chiariello, L, Gioffre, P.A, 1999. Ischemic preconditioning in humans: models, mediators, and clinical relevance. Circulation 100, 559-563.

Torok, R., Konya, J.A., Zadori, D., Veres, G., Szalardy, L., Vecsei, L., Klivenyi, P., 2015 MRNA expression levels of PGC-1alpha in a transgenic and a toxin model of Huntington's disease. Cell. Mol. Neurobiol. 35, 293-301.

Wiegand, F., Liao, W., Busch, C., Castell, S., Knapp, F., Lindauer, U., Megow, D., Meisel A., Redetzky, A., Ruscher, K., Trendelenburg, G., Victorov, I., Riepe, M., Diener, H. C., Dirnagl, U., 1999. Respiratory chain inhibition induces tolerance to focal cerebral ischemia. J. Cereb. Blood Flow Metab. 19, 1229-1237.

Wu, T., Hallett, M., 2013. The cerebellum in Parkinson's disease. Brain 136, 696-709. Zhang, Y., Huypens, P., Adamson, A.W., Chang, J.S., Henagan, T.M., Boudreau, A. Lenard, N.R., Burk, D., Klein, J., Perwitz, N., Shin, J., Fasshauer, M., Kralli, A. Gettys, T.W., 2009. Alternative mRNA splicing produces a novel biologically active short isoform of PGC-1alpha. J. Biol. Chem. 284, 32813-32826.

Zheng, B., Liao, Z., Locascio, J.J., Lesniak, K.A., Roderick, S.S., Watt, M.L., Eklund, A.C. Zhang-James, Y., Kim, P.D., Hauser, M.A., Grunblatt, E., Moran, L.B., Mandel, S.A. Riederer, P., Miller, R.M., Federoff, H.J., Wullner, U., Papapetropoulos, S., Youdim, M.B., Cantuti-Castelvetri, I., Young, A.B., Vance, J.M., Davis, R.L., Hedreen, J.C., Adler, C.H., Beach, T.G., Graeber, M.B., Middleton, F.A., Rochet, J.C., Scherzer, C.R Global PD Gene Expression (GPEX) Consortium, 2010. PGC-1alpha, a potential therapeutic target for early intervention in Parkinson's disease. Sci. Transl. Med 2, 52 ra73. 